Portland State University

PDXScholar

6-10-2020

\title{
Weekend Spanish Immersion Camp: a Non- Traditional Teaching World Language to Middle School American Students
}

Jorge Oscar Ramirez

Portland State University

Follow this and additional works at: https://pdxscholar.library.pdx.edu/open_access_etds

Part of the Curriculum and Instruction Commons, and the First and Second Language Acquisition Commons

Let us know how access to this document benefits you.

\section{Recommended Citation}

Ramirez, Jorge Oscar, "Weekend Spanish Immersion Camp: a Non-Traditional Teaching World Language to Middle School American Students" (2020). Dissertations and Theses. Paper 5476.

https://doi.org/10.15760/etd.7348

This Dissertation is brought to you for free and open access. It has been accepted for inclusion in Dissertations and Theses by an authorized administrator of PDXScholar. Please contact us if we can make this document more accessible: pdxscholar@pdx.edu. 
Weekend Spanish Immersion Camp: A Non-Traditional Teaching World Language to Middle School American Students

\author{
by \\ Jorge Oscar Ramírez
}

\begin{abstract}
A dissertation submitted in partial fulfillment of the requirements for the degree of
\end{abstract}

\author{
Doctor of Education \\ in \\ Educational Leadership: Curriculum and Instruction
}
Dissertation Committee:
Yer J. Thao, Chair
Joanna E. Cooper
Alex Xander Davies
Roberto de Anda

Portland State University

2020 


\begin{abstract}
The nature of today's global economy demands that students demonstrate high levels of proficiency in foreign languages. To meet global demand, most countries around the world are including comprehensive world language instruction in their educational systems beginning at an early age. The increasingly multi-lingual nature of culture within and beyond the Unites States. is escalating the importance of immersing students in world languages as early as possible to ensure a high level of written and oral foreign language skill proficiency by the time students graduate from high school. However, most American students do not have the opportunity to study a world language before middle school and often not until they are in high school. Thus, most students graduating from public schools in the Unites States lack the same level of foreign language proficiency found in similar countries such as Canada to the north. A key controversial issue in the field of world language education in the United States is related to appropriate timing and intensity of students' exposure to learning a foreign language. The approach to world language instruction currently provided in the public middle schools in the United States is not enough to become proficient in two basic foreign language skills; speaking and understanding. Supplemental immersion experiences have been found to enhance proficiency levels, however there is little student-centered research that describes students' perspective on the value of camp activities. Therefore, I conducted a qualitative participatory observer action research study focused on a fourweekend Spanish immersion camp involving a group of middle school students in their
\end{abstract}


second year of world language instruction to elicit and describe how, if at all, campers perceive that camp experiences contributed to the retention and development of their Spanish language skills. This study found that the immersion experience significantly improved students' language. Students appreciated the practical nature of the camp that required the use and development of conversational Spanish skills as a vehicle to increase their world language communicative skills. Each activity provided throughout the Weekend Spanish Immersion Camp was planned to entertain students while directly supporting language usage. The practical nature of the camp's structure reinforced the importance of what they were learning because they saw for themselves how their new found knowledge could be used both within and outside the classroom setting. 


\section{Dedication}

This work is dedicated to my wife, Nancy and my children Kenneth, Sidney and Lindsey for the support, encouragement, and love they have demonstrated all these years, especially during the last stage of my education. May this work be a family triumph. May this work be an example of courage and perseverance.

I also want to dedicate this accomplishment to my mother Roberta and my brothers and sisters. A special thanks to my sister Teresa and her husband Greg for giving my family and I the opportunity to immigrate to the United States. Moreover, I want to dedicate this dissertation to my family and friends in Peru. Now I can say "I have reached the American dream". Thanks to all of you. 


\section{Acknowledgments}

I would like to thank my committee members who were more than generous with their support, advise, expertise and time. A special thanks to Dr. Yer Thao, my advisor, for his unconditional support and encouragement. He has dedicated many hours of his precious time of reading, reflecting, and providing me with prompt feedback on my dissertation. I also want to thank my dissertation committee members for making a time in their busy work schedule to read, analyze, and provide me with wise and professional comments and advise.

I want to acknowledge my school students for their participation in my research project. Thanks for attending the four weekends dedicated to school, and for being an important part of my dissertation. Without them, this study could not have been accomplished.

I also want to thank my school district administration for allowing me to conduct my research and providing me with any assistance I needed and requested. Moreover, I would like to thank the language coaches, and other staff who took part on this project. The excitement, willingness, and effort they provided helped me reach my initial goal.

Finally, I would like to thank Dr. Jenifer Wells, my editor, for her expertise and professional guidance during this dissertation. I do not want to conclude without acknowledge Sidney and Lindsey Ramirez, my daughters, for their involvement and constant support from the very beginning to the end of this dissertation. Their encouragement and support made this journey an unforgettable and enjoyable experience. 


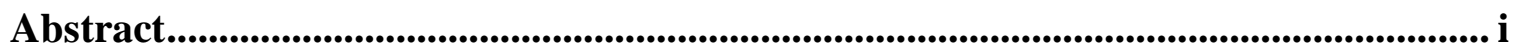

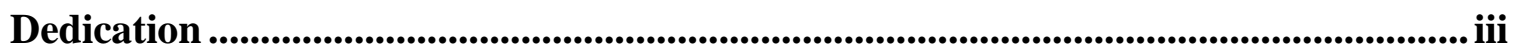

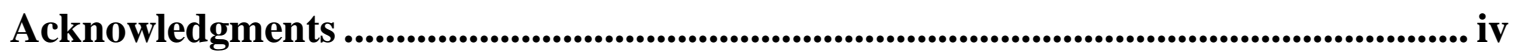

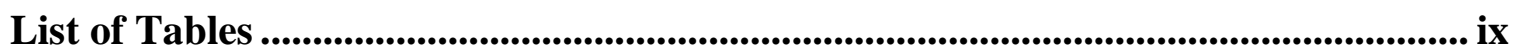

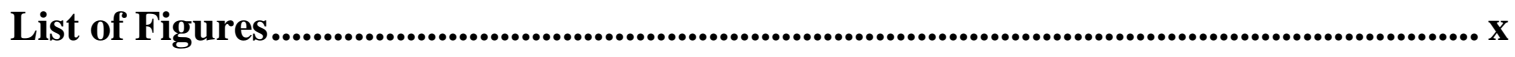

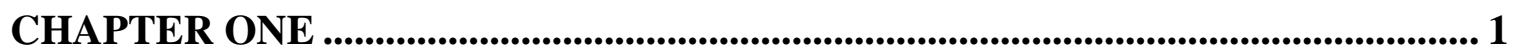

THE PROBLEM ............................................................................................................ 1

Background of the Problem .......................................................................................... 4

Context of the Problem ............................................................................................................. 4

The Impact of World Language in the Unites States..........................................8 8

Key Issues Related to Improving World Language Education .......................... 11

The Inequity in Funding World Language Programs and Academic Resources

Statement of the Research Problem ................................................................................... 18

Significance of the Research Problem ................................................................... 20

Presentation of Methods and Research Questions...................................................... 24

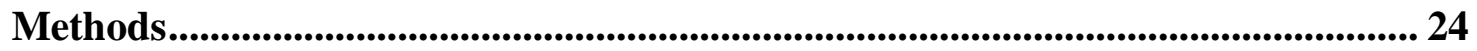

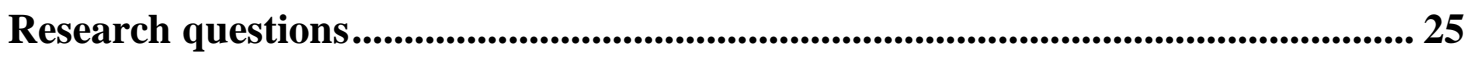

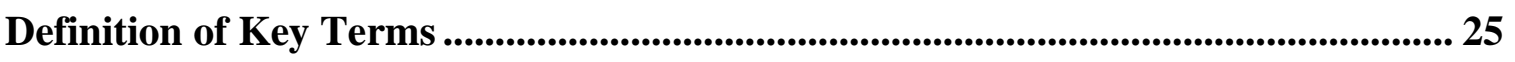

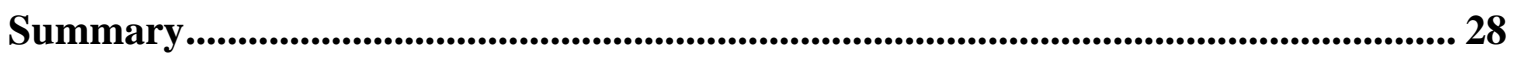

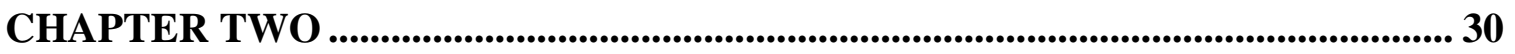

LITERATURE REVIEW ...................................................................................... 30

Theoretical Frameworks .................................................................................................. 32

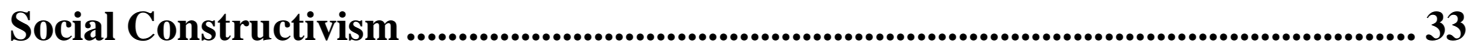

Jean Piaget's Social Constructivism. ................................................................. 33

Analysis of the problem...................................................................................... 35

Critique of Jean Piaget's Social Constructivism. ...................................................... 36

Vygotsky's Description of Social Constructivism. .................................................... 37

Analysis of the problem through the lens of Vygotsky's Interpretation of Social Constructivism.................................................................................................... 38

Critique of Social Constructivism...................................................................... 39 
Situated Learning Theory ............................................................................................................. 40

Analysis of the Problem........................................................................................................ 42

Critique of the Situated Learning Framework .......................................................... 45

Literature Review of Immersion Programs ........................................................................ 46

History and Objective of Foreign Language Immersion Programs......................... 46

Types of Immersion Programs ............................................................................................... 48

Two Way Immersion (TWI) or Dual Language Program (DLP) Models......... 50

World Language Immersion Camps............................................................................. 52

World Language Immersion Camp Instructors ..........................................................5 53

World Language Immersion Camp Programs and Community.................................55

Synthesis of the Research Literature ......................................................................5 56

Critique of the Research Literature ..........................................................................5 59

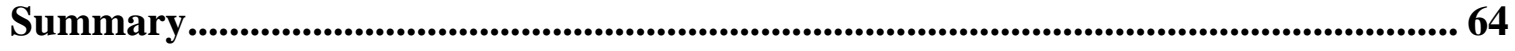

CHAPTER THREE ........................................................................................... 66

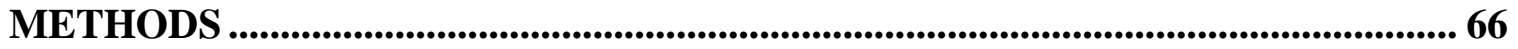

Research Methods................................................................................................................ 66

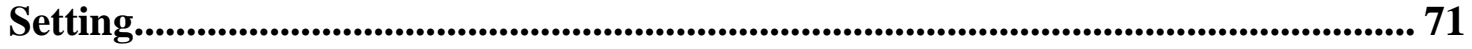

Description of the Spanish Immersion Camp ............................................................ 71

Learning Activities............................................................................................................ 71

Physical activities. ........................................................................................................ 71

Cultural Activities......................................................................................................... 72

Participants................................................................................................................. 73

Staff. ................................................................................................................ 73

Students........................................................................................................................ 74

Participant recruitment ............................................................................................. 76

Participant Selection ........................................................................................................... 77

Protection of Human Subjects ............................................................................................ 77

Instruments and Measurements .......................................................................................... 78

Data Collection and Analysis ........................................................................................... 79

Analytical Stages .......................................................................................................................... 80

Reduction or Breakdown. ....................................................................................... 81

Exploration of Text. ................................................................................................................. 81 
Integration of Exploration. ............................................................................................. 82

Data Maintenance ............................................................................................................. 82

Role of the Researcher ....................................................................................................... 83

Potential Threats to Validity .................................................................................................. 85

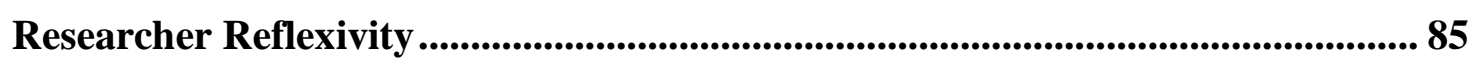

Member Checking..................................................................................................................... 85

Peer Debriefing........................................................................................................................... 86

Participatory Observer Action Research Timeline ............................................................ 88

Summary ................................................................................................................................. 90

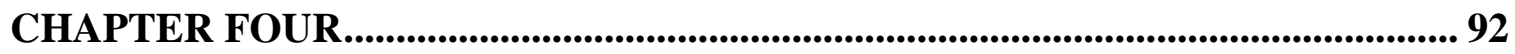

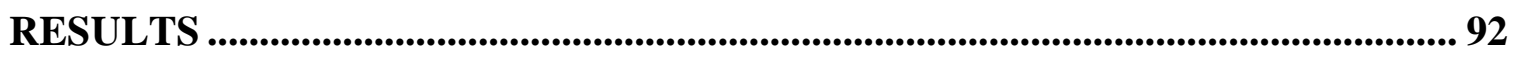

Background of the Study .................................................................................................... 92

Data Analysis and Interpretation ...................................................................................... 94

Pre-camp Questionnaire........................................................................................... 94

Camp Observations .............................................................................................................. 100

Interviews............................................................................................................................... 103

Presentation of Results .......................................................................................................... 103

Interview Data that Informs the First Research Question........................................... 104

Data that Informs the Second Research Question ........................................................ 111

Activities Students Preferred. ......................................................................................... 111

Activities Students Did Not Prefer. ............................................................................ 114

What Students Liked About the Preferred Activities. ........................................... 116

Data that Informs the Third Research Question .......................................................... 117

Summary of Findings from the Interviews................................................................... 122

Summary of Findings Related to Question One........................................................ 122

Summary of Finding that Inform the Second Research Question..................... 124

Summary of findings that Inform the Third Research Question....................... 127

Limitations of the Study .......................................................................................................... 129

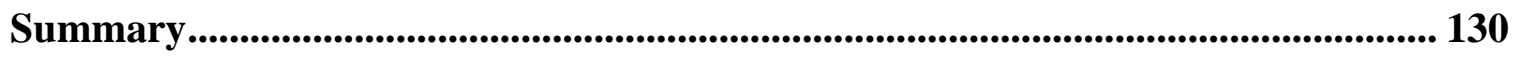

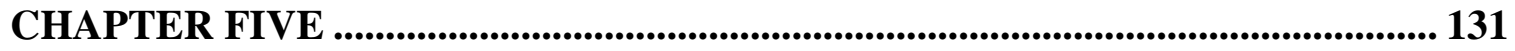

DISCUSSION AND CONCLUSION ......................................................................... 131

Analysis of Findings................................................................................................................. 133 
Themes that Informed the First Research Question ..................................................... 133

Themes that Inform the Second Research Question .................................................... 136

Themes that Inform the Third Research Question..................................................... 140

Implications .................................................................................................................................. 142

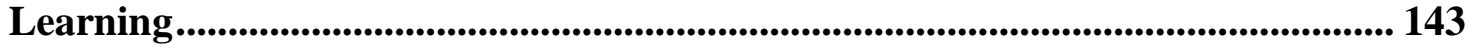

Behavior and Relationships ............................................................................................... 146

Self-confidence................................................................................................................. 147

Recommendations for Future Research ....................................................................... 149

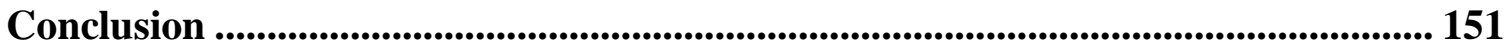

References ........................................................................................................................ 154

Appendix A: Invitation to the Research .......................................................................... 162

Appendix B: Consent and Release....................................................................... 164

Appendix C: Camp Information Package ......................................................................... 166

Appendix D: Parent Application Form....................................................................... 169

Appendix E: Student Application Form ................................................................... 170

Appendix F: Student Information.................................................................................... 171

Appendix G: Pre-Camp Questionnaire ....................................................................... 172

Appendix H: Interview Protocol........................................................................................... 173

Appendix I: Camp Schedule .................................................................................................. 174 


\section{List of Tables}

1. Table 1: Demand for Educators Survey, regions survey.................... 14

2. Table 2: Educator demand by region and certification....................... 15

3. Table 3: Weekend Spanish Immersion Camp staff...........................73

4. Table 4: Weekend Spanish Immersion Camp students........................75 


\section{List of Figures}

1. Figure 1: The Action Research Cycle......................................68

2. Figure 2: Thematic networks: an analytic tool for qualitive research.............80 


\section{CHAPTER ONE}

\section{THE PROBLEM}

Immigrants are present all over the world (Burn \& Perkins, 1980; Valdés, 1995), and the United States of America is certainly no exception. In fact, society in the United States is becoming increasingly culturally pluralistic (Valdés, 1995; Goldman 2018). Despite this fact, American society continues to remain largely monolingual and in need of improved models of foreign language skills development. American students need to become bilingual and bicultural, therefore, they need to have access to authentic world language education in the Unites States.

Furthermore, given the increasingly multilingual nature of American society, our ability to navigate world language proficiency affects what we accomplish in our organizations and how well our students learn in our schools. Indeed, the acquisition and development of world language communication skills are essential in today's world. Multilingual and multicultural proficiencies are essential to helping immigrants successfully adapt into schools and the broader culture, thereby enhancing our domestic well-being and promoting internal and international cooperation.

For example, providing world language education will contribute to Americas' capacity in term of ensuring our national security (Pufahl \& Rhodes, 2011). This is not new information, in fact experts have been pointing to this fact for some time. In 1980 Burn and Perkins wrote "Our foreign policy has become much more closely intertwined with domestic issues among which cultural pluralism is important. In an interdependent world, we cannot solve problems independently, but must work in concert with other 
nations" (p. 17).

In the thirty-nine years since Burn and Perkins (1980) wrote this strong statement, the points they made have become ever increasingly true. For example, the creation of a peaceful learning environment in our schools and greater society is dependent upon a high degree of multicultural competency that promotes social understanding within our nation. Our students' ability to speak and understand a world language and awareness of foreign cultures allows them to develop more empathic relationship with immigrants.

However, broadly speaking, most Americans are not highly proficient in the use of languages beyond their native language. Given the fact that "...fewer than half of high school students are enrolled in a world language class" (Wallis \& Steptoe, 2006, p. 2), it is apparent that the educational system in the Unites States is simply not responding to the pressures of globalization in a sufficient manner. Pufahl and Rhodes (2011) pointed out that American stakeholders, including many educators, do not recognize the vital importance of the ability to communicate in languages other than English; subsequently they do not appreciate the cognitive, social, and academic benefits of a world language education. This may partially explain the cause of inequitable access for students to world language instruction in both public and private school settings, inequities that are more pervasive in rural schools as compared to urban schools in the Unites States.

Wallis and Steptoe (2006) argued that American education is not adapting as rapidly to research that points to best practices as are other sectors in the Unites States Public schools tend to be exactly like they were years ago; students are seated in rows, taking notes from teachers' instruction, and reading from outdated books. This obsolete, 
but still common classroom practice limits interaction between students, limits the expression of diverse perspectives, and therefore disconnects classrooms from the world outside school walls.

Pufahl and Rhodes (2011) found that intensive, immersive instruction in the target language and a high degree of student interaction produce higher levels of language proficiency in comparison to traditional direct instruction models. However, according to their analysis, "The immersion model — the only model that consistently provides instruction that allows students to attain a high level of proficiency — was offered by only $6 \%$ of public schools with language programs" (p. 274).

The typical foreign language immersion model involves primary and secondary level instruction of the core academic subjects in the target language (Pufahl \& Rhodes, 2011). However, while this model could be applied to the primary level where students typically receive instruction of the core academic subjects from only one teacher, secondary level students usually have multiple teachers for multiple subjects. Thus, implementation of the immersion model at the secondary level would involve retraining all teachers, incapable of instructing their core academic subject in both English and the target foreign language or replacing instructors with teachers who have a high level of language proficiency in both English and the target foreign language. Given that this is an unreasonable solution for immediate implementation, supplemental instruction provided by instructors with dual language proficiency is necessary to offer language immersion programming in schools.

One supplemental foreign language education model used in schools across the 
nation are weekend language immersion camps provided on the school grounds. The rational for providing world language immersion camp programs in the Unites States is that public middle and high school students are not receiving enough world language instructional time during regular school hours to become highly proficient in a world language by graduation. However, little current research exists that describes a studentcentered perspective on the effectiveness of immersion camp structures and activities on students' development of foreign language skills.

In response to this gap, I conducted a study that contributed towards the development of a baseline of understanding of middle school level students' perceptions in response to the structure and activities of a four-weekend Spanish language immersion camp model. The intention of this dissertation study was to contribute towards the development of a foundation of understanding necessary to support evidence-based development of weekend Spanish Immersion Camp intervention programs.

The next section will provide a brief overview of the background of the problem. Here, I begin by providing a description of the context of the problem.

\section{Background of the Problem}

\section{Context of the Problem}

As mentioned above, the modern economy challenges citizens to be exposed to other cultures and be able to speak and understand different languages to be part of the new global economy. "Minority languages and minority language speaking populations are present in countries all over the world" (Valdés, 1995, p. 321). Moreover, "This population continues to expand across the continent" (Goldman, 2018, p. 01). World 
language instruction is necessary in all schools because the modern global economy moves business around the world and therefore moves families.

Indeed, Pufahl and Rhodes (2011) stated that learning world language is as important as other academic areas. Possessing bilingual skills is advantageous for business, education, research, technology, medicine, and tourism. Moreover, Wallis and Steptoe (2006) said that “today's economy demands not only a high-level of competence in the traditional academic disciplines but also what might may be called 21 st century skills...kids are global citizens now" (p. 2).

Yet demand for foreign language instruction is outpacing the resources available to students. Burn and Perkins (1980) argued that the need to provide world language instruction to all students is increasing for kindergarten through Grade twelve (K-12) and for higher education levels. To maintain economic power on the world stage, governments must spend money to provide world language education from K-12 through higher education. Moreover, Burn and Perkins (1980) indicated that most American business are in need of staff knowledgeable in foreign languages and international studies. The demand for employees who can speak more than one language is increasing every day as companies are looking for professionals able to communicate in world languages to increase the possibilities for their businesses to expand to distant frontiers. Pufahl and Rhodes (2011) said that most developed countries are offering world language education to their student populations at an early age. However, world languages are typically only offered as elective classes at the high school level in most 
school districts in the Unites States. Thus, students may graduate from high school with up to four years of world language instruction only in the best-case scenarios.

Contemporary school districts are seeking bilingual teachers able to instruct their diverse student population using both English and a world language. However, despite evidence that points to the benefits of early foreign language immersion experiences, only $58 \%$ of elementary world language teachers use a target foreign language as a key part of their immersion instructional strategy in the Unites States (Pufahl \& Rhodes, 2011). Furthermore, according to Pufahl and Rhodes (2011), only 79\% of high school teachers and $81 \%$ of middle school teachers they surveyed used world language to communicate during instruction within the context of world language courses. One reason for this may be that elementary level teachers lack advanced foreign language proficiency themselves and therefore are limited in the level of conversational skills they are able to employ in the classroom. However, significant increases in immigrant populations who speak English as a second language and evidence that immersive language experiences have positive impacts on student learning have increased the demand for preparing fully bilingual teachers (Valdés, 1995). Moreover, Cummins (2017) indicated that educators are using bilingual instructional strategies to increase academic language development.

In an effort to increase the number of years of world language instruction offered to students in the Unites States, some school districts started offering world languages at middle school level as elective classes. However, as Pufahl and Rhodes have pointed out (2011), this is not occurring on a wide spread basis because broad sectors of politicians 
and educators lack clear understanding that learning a world language increases students' cognitive perspective in terms of learning how to speak and understand a world language and learning about foreign cultures thus expanding their worldview beyond their own limited cultural milieu. Plus, many decision-makers lack awareness that learning a foreign language enhances students' native language skills due to increased consciousness of their use of grammar, idioms, and sentences construction in any language (Prevoo, Malda, Mesman, \& van IJzendoorn, 2015).

According to Valdés (1995), Spanish is one of the most popular world languages offered at the elementary, middle, high school, and university levels in the Unites States.

Likewise, Goldman (2018) stated that the estimated immigrant student population is over 5 million and Spanish speaking population is the largest representation. These authors claimed that there is a need to offer authentic Spanish language immersion support to public middle and high school students to increase oral and comprehension skills to communicate with a significant portion of citizens within the Unites States who speak Spanish as their first language.

Ennis, Ríos-Vargas, and Albert (2015) explained that "Hispanic or Latino refers to a person of Cuban, Mexican, Puerto Rican, South or Central America or other Spanish culture or origin regardless of race" (p. 2). These researchers recently conducted a study that found that "more than half of the growth in the total population of the United States between 2000 and 2010 was due to the increase in the Hispanic population" (Ennis, et al., 2015, p. 2). Indeed, 2010 Unites States census data indicated that the Hispanic or Latino ethnicity population increased to 50.5 million people out of 308.7 million people living in 
the Unites States. In fact, overall in the Unites States the number of Spanish speakers grew dramatically between 1980 and 1990. Ennis, Ríos-Vargas, and Albert (2015) pointed out that "the numbers of Spanish speakers increased from 11.1 million persons in 1980 to 17.3 million in 1990 when they represented $54.5 \%$ of the non-English speaking population in the United States” (p. 2).

This growth in the number of people who speak Spanish as their first language is significantly impacting schools. Indeed, the school-age segment of the population grew by 1.2 million persons at a rate of $41.4 \% "$ (Valdés, 1995, p. 303). This phenomenon has especially impacted “California, Texas, New York, Florida, Illinois, New Jersey, Arizona, New Mexico, Colorado, and Massachusetts" (Valdés, 1995, p. 303).

Because the Spanish speaking population has increased, the need to assist this population forces many school districts to focus their attention on Spanish language instruction more than on other world languages. I argue that this significant change in the national demographic creates a need to shift the level of priority of world language instruction in Unites States public secondary schools and that foreign languages instruction should become a core subject class to provide enough time for learners to develop high levels of language proficiency by graduation.

\section{The Impact of World Language in the Unites States}

A study conducted by Rubinstein-Avila (2017) explained that the Unites States is a place of mass global migration. Notwithstanding the commonly held belief that America is a nation of immigrants, it is very difficult to enter, live, and work in the Unites States as a modern immigrant. In order to assist people with successfully 
navigating immigration it is imperative to reflect on the challenges implicit to immigration both for those who personally experience immigration and for the communities where people live, work, learn, and play together. The range of challenges related to immigration within the Unites States are many. Here I will highlight two powerful examples that point to the need to produce more graduates from Unites States schools well prepared to communicate with people who do not speak English or speak English as a second language.

One of the biggest problems government offices face is the need for bilingual employees or translator support. As is the case for many nations, most Unites States agencies "are monolingual in an official sense" (Valdés, 1995, p. 301), however most immigrant citizens use their native languages, which in most cases is not the English language. Valdés (1995) pointed out that the Unites States is not the only developed country that primarily relies on using the official language of the nation to conduct the majority of their interactions. She said:

Their governmental institutions employ only the dominant or designated national language in all interactions with its citizenry. Ordinarily such monolingual nations have tended to ignore the special needs and circumstances of its immigrant and indigenous linguistic minorities who for numerous reasons carry out their lives using two languages. (Valdés, p. 301)

While Unites States agencies are not completely ignoring the special needs of linguistic minorities, the number of staff members available to work with those who speak English as a second language is quite limited. For example, members of immigrant populations often require a translator while interacting with monolingual staff in 
government offices. This scenario places the government in a position wherein they must hire private interpreters or bilingual employees to assist immigrant populations.

There are a limited number of well qualified people to hire who speak foreign languages, consequently it is difficult to meet increasing demands for this and other governmental services. Therefore, to provide people with equitable access to governmental services, the Unites States school system must prepare K-12 and college students to be fluent in world languages to meet this labor demand.

Another issue related to world language are the collateral consequence of the increasingly global economy. Globalization is creating the need for a bilingual workforce ready and able to communicate with multilingual people in nations where companies are conducting business. Even businesses that maintain their base of operations within the Unites States borders are in need of bilingual staff to communicate with customers who speak foreign languages within the Unites States and to increase the number of relationships and commerce with domestic suppliers and those beyond our borders.

When discussing improvements regarding world language instruction, the conversation typically involves calls for the government to find a way to increase educational funding to provide higher quality and earlier world language instruction to students. However, research has demonstrated that early education programs are not increasing opportunities for world languages instruction but in fact, are doing quite the opposite. 
Pufahl and Rhodes (2011) said that, "In 2008, the Center for Applied Linguistics surveyed more than 5,000 public and private schools to gather data for both national and regional profiles of foreign language instruction" (p. 259). These researchers noted that the results of this survey demonstrated a decrease in world language instruction in elementary schools as compared to the last decade. This decrease was from $25 \%$ to $15 \%$ in public schools. A similar scenario appears at the middle school level; the percentage of students receiving world language instruction decreased from $75 \%$ to $58 \%$ between 2000 and 2010, while high schools saw a slight increase from 90\% to 91\%" (Pufahl \& Rhodes, 2011, p. 259).

Some of the reported causes for the decrease in world language instruction for elementary, middle, and high schools included a lack of funding, decision-making at the school district level tends to prioritize English-only education programs, world languages are not seen as a core curricular component, and a shortage of highly qualified world language teachers available to teach foreign language courses let alone core courses in target languages. While the survey results did indicate that Spanish is the most frequent world language offered at the elementary, middle, and high school levels, world language is not seen as a core subject. Consequently, the support of world language instruction in elementary, middle and high school is considered to be a lower priority in the Unites States.

\section{Key Issues Related to Improving World Language Education}

Researchers such as Pufahl and Rhodes (2011), Peyton (1997), Cuseo (2007), LoCastro (1989), and organizations such as the American Association for Employment in 
Education (AAEE), (2016) have identified a number of key issues that require analysis to inform improvements to world language education. These issues are related to world language proficiency levels, teacher shortages, class sizes, and increases in teachers' workloads. "Despite some positive developments, overall foreign language instruction has decreased over the last decade and the achievement gap has widened" (Pufahl \& Rhodes, 2011, p. 258).

Peyton (1997) narrowed the focus of challenges in the field of world language instruction to two key factors: class size increases and shortage of qualified teachers. A key contributor to the problem of large class size and decreased number of foreign language courses offered at lower grade levels is a critical shortage of world language teachers across the Unites States (Pufahl \& Rhodes, 2011). Indeed, every year, school districts are facing difficulties hiring and retaining qualified world language teachers.

Although the problem appears to be more acute in rural areas, the problem of shortages of well qualified world language teachers is widespread in the Unites States. Pufahl and Rhodes 2011 survey of 5,000 school districts across the Unites States found $25 \%$ elementary and $30 \%$ secondary schools reported being impacted by teacher shortages. This result implies that there is a need to increase the number of well qualified world language teachers across the Unites States.

Unfortunately, due to rapid population increases in some regions of the Unites States, school administrators have decided to reduce foreign language instruction at the lower levels and increase the number of students per class in high schools due to teacher shortages despite the fact that increasing class size makes world language instruction 
more difficult to teach and to learn. Cuseo (2007) indicated that large class size leads to lecture-centered style classroom interactions. This environment encourages students to be less involved in the learning process because it reduces the frequency and intensity of teacher-student interactions, student-to-student interactions, and reduces the depth of student thinking. These phenomena tend to result in lower academic achievement and performance (Cuseo, 2007).

For example, LoCastro (1989) stated that in large classes teachers experience significantly increased workloads such as the complexity of lesson preparation and delivery and the sheer number of completed assignments to correct. This situation leaves teachers with less time and opportunity to provide prompt individualized feedback to students. These increases in the workload make it more difficult for teachers to monitor individual student's objective achievement and tailor instructional responses to meet each student's needs. At the same time, students have less opportunity to be active participants in class and to demonstrate or correct their understanding of content knowledge in a timely manner.

The American Association for Employment in Education (2016) conducted research across the Unites States and received feedback from professionals and educators from colleges, universities, and school districts about the demand for educators and K-12 teachers. Table 1 shows 11 regions across the Unites States, however only ten were consider for this research based on survey return rates. 
Table 1

Demand for Educators Survey, Regions surveyed

\begin{tabular}{|c|l|c|c|}
\hline \multicolumn{1}{c|}{ Code } & Reglon & Colleges/ Unlversities & School Districts \\
\hline 1 & Northwest & 7 & 13 \\
\hline 2 & West & 10 & 101 \\
\hline 3 & Rocky Mountain & 4 & 33 \\
\hline 4 & Great Plains/Midwest & 23 & 23 \\
\hline 5 & South Central & 21 & 22 \\
\hline 6 & Southeast & 42 & 75 \\
\hline 7 & Great Lakes & 33 & 43 \\
\hline 8 & Middle Atlantic & 45 & 57 \\
\hline 9 & Northeast & 6 & 12 \\
\hline 10 & Alaska & 1 & 1 \\
\hline 11 & Hawaii & 0 & 0 \\
\hline
\end{tabular}

Note: From American Association for Employment in Education (2015-2016).

The survey asked regions to rate 59 certification/subject areas on a scale of oneto-five, with one meaning that the state reported no need for new teachers and five being in considerable need for new teachers. Of the 59 certification areas listed, colleges and universities reported 11 areas as having considerable shortage, while school districts indicated considerable shortage in 17 areas (American Association for Employment in Education, 2016). Both colleges and universities and school districts reported significant shortage in the following ten disciplinary areas: Bilingual/Multicultural Education, Physics, Chemistry, Math, Dual Certification (General \& Special Education), Hearing Impaired Special Education, Visually Impaired Special Education, Mild/Moderate Disabilities Special Education, Multi-categorical Special Education, and Severe/Profound Disabilities Special Education.

Table 2 below depicts that foreign language, math, science, and special education continue to be shortage areas in education across all regions. Note that the third row 
refers to bilingual education.

Table 2

Educator demand by region and certification

\begin{tabular}{c|c|c|c|c|}
\hline $\begin{array}{c}\text { Considerable } \\
\text { shortage }\end{array}$ & Some shortage & Balanced & Some surplus & Considerable \\
surplus \\
$(4.21-5.00)$ & $(3.41-4.20)$ & $(2.61-3.40)$ & $(1.81-2.60)$ & $(1.00-1.80)$ \\
\hline
\end{tabular}

\begin{tabular}{|c|c|c|c|c|c|c|c|c|c|c|c|c|}
\hline & & 1 & 2 & 3 & 4 & 5 & 6 & 7 & 8 & 9 & 10 & Total \\
\hline \multirow{17}{*}{ 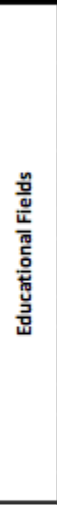 } & Agriculture Education & 4.25 & 3.80 & 4.29 & 4.54 & 4.04 & 3.72 & 4.25 & 3.94 & 4.00 & & 3.96 \\
\hline & Art/Visual Education & 3.29 & 3.47 & 3.09 & 3.06 & 2.90 & 2.95 & 3.12 & 2.64 & 2.85 & 5.00 & 3.07 \\
\hline & Bilingual Education/Multicultural & 4.50 & 4.16 & 4.28 & 4.23 & 4.74 & 4.32 & 4.38 & 4.06 & 4.73 & 3.00 & 4.29 \\
\hline & Business Education & 3.36 & 3.27 & 3.52 & 3.06 & 3.09 & 3.04 & 3.60 & 3.06 & 3.25 & & 3.21 \\
\hline & Computer Science Education & 3.80 & 3.84 & 3.65 & 3.23 & 3.46 & 3.44 & 3.93 & 3.36 & 3.73 & 3.00 & 3.60 \\
\hline & Dance Education & 3.57 & 3.33 & 3.21 & 3.17 & 2.88 & 3.29 & 3.07 & 3.21 & 3.00 & & 3.22 \\
\hline & English/Language Arts Education & 3.06 & 3.27 & 3.09 & 2.91 & 3.39 & 3.16 & 2.82 & 2.49 & 2.00 & 4.00 & 2.97 \\
\hline & Family and Consumer Science & 3.54 & 3.60 & 3.88 & 4.20 & 3.42 & 3.54 & 3.69 & 3.94 & 3.86 & & 3.71 \\
\hline & Health Education & 2.64 & 3.10 & 2.66 & 3.00 & 2.48 & 2.62 & 2.74 & 2.31 & 3.08 & 4.00 & 2.72 \\
\hline & Journalism Education & 3.43 & 3.34 & 3.19 & 3.27 & 3.08 & 3.04 & 3.31 & 2.81 & 3.00 & 4.00 & 3.18 \\
\hline & Music Education & 3.60 & 3.74 & 3.09 & 3.38 & 3.11 & 2.87 & 2.92 & 2.78 & 2.85 & 5.00 & 3.14 \\
\hline & Physical Education & 2.62 & 2.69 & 2.30 & 2.03 & 2.14 & 2.34 & 2.32 & 2.00 & 2.55 & 3.00 & 2.33 \\
\hline & Reading Specialist/Interventionist & 3.60 & 3.71 & 3.44 & 3.57 & 3.21 & 3.55 & 3.57 & 3.43 & 4.00 & 4.00 & 3.55 \\
\hline & Social Studies Education & 2.40 & 2.76 & 2.26 & 2.11 & 2.81 & 2.42 & 2.14 & 1.78 & 1.69 & 3.00 & 2.30 \\
\hline & Speech Education & 4.42 & 4.39 & 3.86 & 3.81 & 3.14 & 4.21 & 4.00 & 4.04 & 4.45 & & 4.08 \\
\hline & Technology Education & 3.43 & 3.92 & 3.50 & 3.58 & 3.44 & 3.70 & 3.98 & 3.62 & 3.82 & 4.00 & 3.71 \\
\hline & Theatre/Drama Education & 3.17 & 3.40 & 3.38 & 3.13 & 3.00 & 2.96 & 3.25 & 3.05 & 3.00 & 4.00 & 3.16 \\
\hline \multirow{8}{*}{ 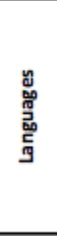 } & Chinese & 4.44 & 4.35 & 4.71 & 4.40 & 4.50 & 4.55 & 4.42 & 4.29 & 4.13 & 1.00 & 4.40 \\
\hline & Classical (Greek, Latin) & 4.80 & 3.96 & 4.50 & 4.09 & 4.00 & 4.33 & 4.20 & 4.25 & 3.82 & & 4.19 \\
\hline & ESL/ELL (English Language Learner) & 4.36 & 3.82 & 4.19 & 4.06 & 4.00 & 4.21 & 4.14 & 3.94 & 4.45 & 3.00 & 4.05 \\
\hline & French & 3.56 & 3.82 & 4.16 & 3.74 & 4.00 & 3.77 & 3.67 & 3.70 & 3.67 & & 3.79 \\
\hline & German & 3.75 & 3.84 & 4.19 & 3.81 & 4.13 & 3.95 & 3.61 & 3.89 & 4.00 & 4.00 & 3.89 \\
\hline & Japanese & 4.33 & 4.00 & 4.45 & 4.18 & 4.50 & 4.44 & 4.24 & 4.17 & 4.25 & & 4.26 \\
\hline & Spanish & 3.76 & 3.68 & 3.94 & 3.79 & 4.37 & 4.07 & 3.71 & 3.76 & 3.86 & 3.00 & 3.87 \\
\hline & Other Languages & 3.86 & 4.00 & 4.15 & 3.88 & 4.21 & 4.12 & 4.38 & 4.19 & 3.50 & & 4.10 \\
\hline \multirow{6}{*}{ 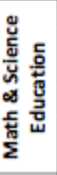 } & Math & 4.67 & 4.64 & 4.60 & 4.23 & 4.45 & 4.67 & 3.87 & 3.94 & 4.50 & 5.00 & 4.37 \\
\hline & Biology & 3.94 & 4.31 & 3.73 & 4.02 & 4.03 & 4.24 & 3.84 & 3.76 & 4.13 & 5.00 & 4.03 \\
\hline & Chemistry & 4.33 & 4.57 & 4.24 & 4.48 & 4.38 & 4.52 & 4.04 & 4.27 & 4.71 & 5.00 & 4.39 \\
\hline & Earth/Physical & 4.00 & 4.29 & 3.61 & 4.08 & 3.94 & 4.24 & 3.69 & 3.75 & 4.22 & 4.00 & 4.00 \\
\hline & General Math and Science & 4.41 & 4.41 & 4.15 & 3.97 & 4.13 & 4.33 & 3.58 & 3.59 & 4.27 & 4.00 & 4.08 \\
\hline & Physics & 4.33 & 4.54 & 4.48 & 4.46 & 4.45 & 4.58 & 4.26 & 4.58 & 4.85 & 5.00 & 4.51 \\
\hline
\end{tabular}

Note: Reprinted from American Association for Employment in Education (2016).

\section{The Inequity in Funding World Language Programs and Academic}

\section{Resources}

A relevant issue of concern is the legacy of No Child Left Behind (NCLB) and

unintended negative effects this legislation had on world language programs, especially

on rural schools. Pufahl and Rhodes (2011) indicated that the NCLB legislative policy

and the implementation of national standards negatively affected world language 
instruction since foreign language instruction was not named as one of the so-called core subjects but rather, was relegated to the status of elective classes. In order to meet school achievement goals as defined by this national legislation, school administrators redirected resources such as funding and instructional time to support classes that maximize students' core subject achievement.

Most school districts in the nation, especially those from rural areas with limited resources, increased funding and instructional time in math, language arts, science, and social science classes to support students to reach high levels of achievement on state standardized tests. This policy effectively cut or reduced the opportunity to offer elective classes, especially world language programs. The lack of funding created inequities in terms of students accessing world language education, especially students from rural areas.

Pufahl and Rhodes (2011) emphasized that inequitable access to world language instruction for students enrolled in rural and low social economic status (SES) schools is a continuing problem. This problem is a consequence of the lack of funding to rural and low socioeconomic (SES) schools resulting in difficulty maintaining quality world language programs that support students to achieve high levels of world language proficiency.

Snow, Padilla, and Campbell (1988) cautioned that world language students begin to forget some of their world language knowledge as soon as formal learning instruction ceases. To maintain and improve language skills and knowledge retention post formal instruction, it is necessary to continuously instill retention strategies and habits. One way 
this can be done is to reinforce language skill retention and development through learning experiences offered outside of the formal education setting. With support, world language students can more easily reach higher levels of proficiency and retain their skills as they move through increasingly advanced learning experiences including those beyond the classroom. However, in adverse circumstances wherein insufficient supports are provided within formal learning situations and in informal situations including at home, the opposite outcome is likely to occur.

Valdés (1995) argued that a significant problem world language teachers face is a lack of academic support at home. A legacy of NCLB is that parents who are underprepared themselves in foreign language skills are unprepared to provide supports to their children learning a foreign language. This is especially problematic in rural and low socio-economic schools where students often do not have the same level of academic support at home as do urban students.

In scenarios where students are lacking home academic support, Valdés (1995) said that school districts should offer alternative educational supports to supplement formal education and home support. Moreover, she indicated that after-school academic resources have been shown to make a difference for some students. Examples of afterschool academic resources are camps or clubs that bring some degree of balance for students who lack academic support at home. Under these circumstances, student motivation has been shown to increase and thus, so have levels of students' world language proficiency achievement (Valdés, 1995). 


\section{Statement of the Research Problem}

The demands of global communication and cooperation challenge graduating students to be world language proficient. Therefore, most countries are including comprehensive world language immersion programs in their education systems beginning at an early age to adequately prepare graduates to meet global demand. However, most students graduating from Unites States public schools lack the same level of world language proficiency found in similar countries such as Canada.

Researchers such as Wallis and Steptoe (2006), Rubinstein-Avila (2017) and Valdés (1995) indicated that there is a need to educate Unites States students to become knowledgeable not only in core subjects but in the area of world language preparation. Moreover, Gardner (1983) and Center for Public Education (2011) stated that learning a world language requires between three-to-seven years of study and should be begin as earlier as possible.

With this in mind, how can world language teachers better support public secondary students to reach foreign language proficiency? I align with Pufahl and Rhodes' (2011) argument that an immersion program approach is the most beneficial educational model to instruct students in world languages.

Snow, Padilla, and Campbell (1988) pointed out the importance of providing supports that help students develop and maintain language skills over time. Pufah and Rhodes (2011) asserted that the language immersion model wherein select, target subject areas are taught in the world language is the best world language model to support high levels of student achievement. To be considered an authentic language immersion 
experience, the target language needs to be used as much as possible as a means of communication when learning in each of the disciplines.

Pufah and Rhodes (2011) stated that world language educators use a wide range of strategies to scaffold students' learning in listening, reading, and oral proficiency areas. Some examples of the many strategies used by world language teacher are; word walls used to teach vocabulary, realia, which are objects or activities used to relate classroom teaching to the real life, role play, simulation, and drama to improve speaking skills, peer teaching collaboration to support listening and speaking skills and music and songs to enhance vocabulary learning. Furthermore, Snow, Padilla, and Campbell (1988) argued that immersion models allow many opportunities for English speakers to develop and maintain world language skills useful for supporting learners' independent language development skills. Immersion models require students to learn in groups and forces them to use the target language to make meaning.

Snow, Padilla, and Campbell (1988) stated that asking students to problem solve and communicate in the target language they are learning has been shown to support the development of students' oral language skills. These are skills that serve them well beyond the actual language instruction experience.

The goal of a language immersion program is "to communicate in the language with a high level of proficiency and acquire an understanding of and appreciation for the other cultures" (Pufah \& Rhodes, 2011, p. 266). Additionally, Cummins (2007) pointed out that many bilingual individuals can negotiate between culture identities more fluidly than a monolingual speaker. These researchers found that being immersed in the target 
language helped students to improve their speaking level because such experiences involve using the target language at all times. In other words, immersion experiences support students to think in the target language they are learning in an increasingly fluid manner. Moreover, learning a world language helps students reflect upon and improve their own native language grammar and therefore, gradually improve their native language skills.

Given the importance of language immersion programs, it is important to carefully describe students' experiences in response to various language immersion models. The significance of this proposed study is that this work will contribute to efforts to address a gap in the literature by providing a description of public secondary world language students' perceptions of their experiences in a particular immersion model. Next, I will describe the significance of the problem in greater detail.

\section{Significance of the Research Problem}

To meet global demand world language should be a high school graduation requirement in the Unites States, but this is not the current situation. According to Oregon State Board of Education (Oregon Department of Education, 2013), high school students need to accumulate a total of 24 high school credits to obtain a diploma. The subjects high school students must study are: mathematics, science, English, history, geography, economics, civic, physical education, health, the arts, and elective classes.

Oregon State Standards state that all students enrolled in public and private schools must take electives classes as a graduation requirement. World language is one of many elective choices that some students have available to them in many schools in Oregon. High school students who choose to study world language as an elective may take two-to- 
four years of world language classes while attending high school.

Despite the efforts world language teachers make during these two-to-four years of instruction, it is not enough time for students to obtain a high enough level of world language proficiency to retain knowledge of the language and continue building upon their knowledge as independent learners. Unfortunately, this situation puts most students in the Unites States at a disadvantage because, as Gardner (1983) argued, this level of world language proficiency requires four-to-six years of study and should be initiated earlier than high school.

If the Unites States wants to educate competitive students prepared with $21_{\text {st }}$ century skills, world language teachers should be looking for new strategies to better support student learning during the brief window of time available to provide world language skills. However, providing opportunities for students to practice use of world language skills in real life situations outside the walls of the classroom is not an easy task to achieve.

Snow, Padilla, and Campbell (1988) explained that most world language class interactions involve learning vocabulary with mechanical exercises and drills. World language teachers play an important role in this matter, but it is difficult for many of them to shift this dynamic since most of their planning time is spent in meetings, grading student work, and class preparations for the day or week ahead. Therefore, it is difficult for many world language teachers to step back and take the time to reconsider their pedagogy and redesign learning experiences to make them more interactive and fun. However, teachers do need to develop lessons involving all the components world 
language skills demands for today's students. World language classes are usually offered in 50-to-55-minute periods (Oregon Department of Education, 2013). During this time, world language teachers must cope with individual learning styles, attitudes, and students' personal interests. Moreover, teachers not only have to teach listening, speaking, reading, and writing skills, but incorporate study of the cultures where the target language is typically spoken.

Snow, Padilla, and Campbell (1988) pointed out is that it is not enough for $21_{\text {st }}$ century students to simply know how to read short passages of basic stories and be able to translate isolated sentences or learn only prearranged dialog. Students must develop the skills to independently interact in formal and informal conversation. They should be able to express their thoughts orally and in writing and understand the culture of the people with whom they interact. However, as Pufah and Rhodes (2011) pointed out, as the situation stands today, the average Unites States high school student does not have enough time or opportunities to build the skills or confidence to use their world language knowledge outside the classroom because of the lack of immersion experiences integrated into their learning process.

As mentioned above, Snow, Padilla, and Campbell (1988) argued that immersion programs are the best learning and practice environment to support the learning of a world language. From my perspective, the aim of learning a world language is to be able to use it in real life situations. According to Snow, Padilla, and Campbell (1988) immersion models allow many opportunities for English speakers to develop the necessary skills to propel oral communication in a foreign language. In other words, 
immersion is a key vehicle to promote oral proficiency. The more time students are immersed in using the target language, the better they are supported to reach a high level of proficiency.

Again, one approach that the teacher can use to immerse and effectively support students' world language achievement is the immersion camp. World language immersion camps serve as an extension of classroom practice. In the case of this study, the world language immersion camp is the perfect excuse for the teachers to use and test a variety of teaching techniques to extend the concepts taught in class within the context of the language and culture to create an environment to support world language learning. The camp environment involves a variety of activities intended to motivate the development of oral language skills through one-on-one and group conversations in Spanish. Examples of these activities include participating in traditional Latin America cooking, shopping, salsa dancing, games, singing, and creative arts. For instance, in the case of the immersion camp described in this study, throughout the cooking activities students were asked to follow directions from a chef who only spoke Spanish, read the ingredient list in Spanish, and use Spanish to negotiate cooking tasks. These activities were intended to create an environment that led students to draw on their knowledge of vocabulary and proper sentence structures to actively communicate using the target language. Importantly, this format was also intended to support increases in students' social confidence, to foster strong relationships between students, and to be fun.

Vines (1983) argued that immersion language camp is a rewarding experience for teachers as well as for students. The different activities used in immersion camps support 
the relationship between staff and students and therefore, increase oral motivation and decrease speaking anxiety. Vines (1983) also stated that world language immersion camps provide students local opportunities to participate directly in a foreign culture without traveling far from home. Here, a world language immersion camp teaching approach supports local students by allowing them to be immersed in target Latin cultures and focus intensely on using the language as it is used in Spanish speaking countries.

\section{Presentation of Methods and Research Questions}

\section{Methods}

This qualitative study was conducted using the participatory observer action research method to examine the perceptions of a small group of Spanish Two, middle school students attending a Spanish immersion camp. The study used the lens of constructivism and situated learning theories to examine how students perceived that the structure and activities of a particular weekend Spanish immersion camp model motivated and supported their world language fluency.

This dissertation describes students' perceptions before, during, and after the Spanish Immersion Camp with special attention paid to their social interactions, language barriers, emotional responses, and language accomplishments. The study provides thick, rich description from a student-centered perspective to inform ways to better scaffold and support students' world language learning through language immersion camp experiences.

Another intended outcome of this effort was to provide descriptions that assists world language staff and administrators to understand how, if at all, providing extra 
curriculum supports benefits students' world language learning and improve the relationship between teachers and their students. Furthermore, analysis of the student response data provides insight to inform similar non-traditional world language educational programs to improve learning in other subject areas.

\section{Research questions}

This qualitative study involved three open ended research questions:

1. To what extent do Middle School Spanish Two students perceive that Spanish immersion camp instruction serves to assist retention of world language communicative skills?

2. What kind of activities from the Spanish immersion camp effectively support Middle School students learning a world language?

3. How does the Spanish immersion camp environment influence Middle School students' perspective of their world language learning process?

\section{Definition of Key Terms}

In this section, important key concepts will be defined and explained. The section begins with a definition non-traditional world language program.

Bicultural people effectively assimilate and participate in two cultures and understand the values and beliefs of both cultures. Bicultural people identify with central elements of their own culture as well as the dominant culture.

Bilingual refers to a person with the capacity of speaking and understanding two different languages fluently. Some people become bilingual because they have acquired 
two languages since childhood while others acquire knowledge of a second language later in life.

Global communication is a term used to provide and share common information with other people across geographic, political, economic, social and cultural frontiers through speaking, listening, and reading. Global communication is vital in business, education, science and diplomacy environments for internal improvement and international relations.

Global culture is broad and complex in its definition because culture is who and what we are in terms of art, language, history, belief, costumes, behavior, rules and laws. Global culture is transmitted by internet, media, and travel. The term culture refers to ways people transmit ideas, meanings, and values within their own community.

Global economy refers to economic activity that takes place across nations. Examples of industries involved in the global economy are banking, agricultural industries, and companies involved in trade of raw materials for manufacturing. The global economy links the economies of nations, therefore an economic crisis for one or more nation typically affect the rest of their trading partners.

Language Immersion Program (IP) is a method of instruction used in bilingual language education. A language immersion program is the fastest way to learn a foreign language. Research conducted by Snow et al. (1988) explained that the effects of immersion programs are that students are able to speak a world language fluently, achieve higher levels of reading comprehension, and broader knowledge of proper grammar in both the target and native languages. The idea of immersing students into a 
new language is a model that is rapidly being adopted in many countries. It is expected that from the beginning of the program, frequency of use of the English language should decrease as students are exposed to hearing and using the target language they are learning. Kearney (2010) explained that the primarily objective of immersion programs is to develop learners' linguistic proficiency and to help students develop positive attitudes toward the language, foreign cultures, and the learning process.

Non-traditional world language education program is a term used to refer to a different way of providing foreign language instruction to student from the typical didactic approach. This term also refers to types of programs that do not use tradition instructional strategies but rather invent new ways of doing things.

The definition of world language will be presented from the perspective of Snow, Padilla, and Campbell (1988). Snow, et al. (1988) used the term to refer to official languages spoken in countries outside the Unites States. In this dissertation, the use of the term world language is used to refer to the non-English languages studied in the Unites States by native English language students.

The term Weekend Spanish Immersion Camp refers to a non-traditional world language supplemental education program offered to world language students wherein students are placed in simulated cultural settings. A camp experience that involves communicative activities specifically designed to scaffold use and understanding of the target language.

Traditional world language education program is also known as conventional world language education. This term refers to long-established customs used in schools 
where traditional teacher-centered instruction is focused on memorization and choral repetition.

\section{Summary}

Chapter one provided background on the topic of why foreign language instruction is important and how weekend Spanish language immersion camp may influence the use and development of students' language speaking skills. It also presented a background of the problem statement and a rationale for this research study, which involves how to support world language in middle school students most effectively. It introduced a brief overview of the methods and research questions that will be used in this study.

World language instruction is as important as core subjects due to global demand and the increasing language diversity (Burn \& Perkins, 1980; Valdés, 1995) in the United States. Culture diversity is increasing every year in the U.S and the need to support this increasing immigrant population is placing increased pressure on governmental agencies and businesses to hire highly proficient bilingual employees.

Researchers such as Gardner (1983) indicate that to be proficient in world language it takes up to seven years of education. Most students in the Unites States receive world language instruction in high school only, therefore, most of our graduated students may complete their education lacking strong world language skills (Wallis \& Steptoe, 2006). Consequently, world language instruction should be offer in earlier grades of education. 
Immersion program is the most effective program to instruct world language (Pufahl \& Rhodes, 2011). The Weekend Spanish Immersion Camp model is one strategy educators may use to support conversational language skills to middle and high school world language students.

Next, chapter two will provide the conceptual framework that supports this study. The chapter presents a literature review of world language immersion programs and the methodology applied in this participatory observer action research. 


\section{CHAPTER TWO}

\section{LITERATURE REVIEW}

In this world where global communication and interaction has become increasingly prevalent, it is imperative to improve and innovate within the Unites States educational system. Challenges that graduates from both public and private schools face are far greater than even before given that it is essential that American high school graduates be globally literate if they are to be competitive on the world stage (Burn \& Perkins, 1980). To be globally literature, students must achieve high levels of proficiency in academic disciplines such as math and science and in what might be called 21 st century global skills (Wallis \& Steptoe, 2006) that include foreign language literacy.

While most countries in the world are preparing and supporting their students with $21_{\text {st }}$ century global skills, the United States (U.S.) public educational system is not prioritizing world language instruction as a core discipline nor adequately providing early and ongoing instruction throughout most students' twelve years of education. The current approach to providing world language instruction in Unites States public schools is insufficient to help students become highly proficient in one or more world languages by high school graduation.

Non-traditional language education environments such as world language immersion language camps represent an innovative approach that can be used to supplement formal instruction of world language knowledge and skill development (Padilla et al, 1988). Researchers Pufah and Rhodes (2011) demonstrated that language immersion programs provide students with practical experiences in using and developing 
high levels of world language proficiency. The purpose of this study is to examine and describe middle school level students' perceptions in response to supplemental instruction designed to improve students' world language oral and written communication skills and comprehension for fluency.

In chapter two I discuss the theoretical framework of constructivism and situated learning that guided this study. Through the lens of these theoretical frameworks I will describe how these constructs were used to examine the problem this study addressed and discuss how the theoretical frameworks apply to the specific context of the problem.

Next, I will present of review of literature relevant to the problem and methodology of the study. I will conclude chapter two with a summary of the research literature and describe how the literature I read relates to the study.

The context of this study was a Spanish language immersion camp that took place over a period of four weekends within the same school that the student participants normally attend during the week. The purpose of the camp was to provide students with the opportunity to develop oral language proficiency by communicating solely in Spanish during each of the four weekend immersion experiences. The goal of the camp was to provide students with an informal learning experience involving the use of conversational Spanish and create new learning opportunities for practical applications of Spanish that went beyond what is typically possible to do within the confines of fifty-minute class periods during the regular school day.

The goal of describing the language immersion camp experience and student responses to this particular Spanish language camp is to provide an example of authentic 
ways to enhance and motivate world language learning and stimulate more productive, adaptive teacher-student relationships. This study broadened the boundaries of current language teaching approaches and provide world language teachers with descriptions of new, fun teaching strategies to use to improve student learning proficiencies they may adapt to fit within their own educational settings.

\section{Theoretical Frameworks}

As mentioned above, the theoretical frameworks used in this study were constructivism and situated learning. I based my interpretation of the theory of constructivism in perspectives rooted in the fields of psychology and sociology. Here, constructivism refers to how people build their knowledge over time based upon their own actions within the environment. The theory is concerned with the cognitive development of the individual, "Thinking, knowing, perceiving, remembering, recognizing, abstracting, generalizing-all these processes are included in the term cognition, which refers to all the intellective activities of the mind" (Spencer, 1971, pg. ix).

"Situated learning theory commends a conceptualization of the process of learning that, in offering an alternative to cognitive theories, departs radically from the received body of knowledge on learning in organizations" (Contu \& Willmott, 2003, p. 283). Human interaction and environment play important roles in learning to speak and understand world languages. According to Platt (2012), setting and students' interaction help develop students' "critical consciousness" (p. 5). Platt (2012) pointed out that by drawing on this consciousness students can build co-creative learning relationships in 
environments that are more powerful for world language achievement than isolated individual learning. Lave and Wenger, (1991) referred to this process of interaction as legitimate peripheral participation.

The reason that I grounded this study within these two frameworks is that I argue that within the context of a language immersion program, student learning is a process in which personal interactions with others is a key element that influences achievement. Students co-construct knowledge and skills based on the immediate demands to use the language as a communication tool rather than as simply words and rules to memorize and recite. Moreover, in the Weekend Spanish Immersion Camp I designed and directed; I posited that students would advance in their oral language proficiencies more quickly than they would without this experience by interacting with same-age peers due to similar social preferences.

Here, I provide a fuller description of constructivism and situated learning. Specifically, I begin with a description of social constructionism.

\section{Social Constructivism}

\section{Jean Piaget's Social Constructivism.}

Piaget defined social constructivism as the process in which people develop knowledge and meaning based on their personal experiences. At the beginning of Piaget's career, he focused on the mechanisms of learning (Fosnot, 1996). For Piaget, people build knowledge based on their own actions within the environment. These actions can be physical or mental (Harlow, Cummings \& Aberasturi, 2007). The human being is in constant transformation because everyone needs to learn and process new 
interpretations that allow them to face everyday situations that have to do with their social environment.

But how does the learning mechanism proposed by Piaget occur? To answer this question, I will describe two key ideas proposed by Piaget later in his career; these are the stages of cognitive development and the concept of equilibrium.

Piaget argued that cognitive development of the individual goes through the following four distinct learning stages:

1) Sensory motor (0-2 years old) - The stage in which children develop body balancing and object manipulation skills. At this stage, it is very common to see children taking any object into their mouth. Danger is not perceived at this stage (Spencer, 1971).

2) Preoperational (2-7 years old) - The stage where language development is essential for reasoning initiation and questioning; what and why? Children at this age are still in the beginning stages of abstract thinking (Spencer, 1971).

3) Concrete operational (7-11 years old) - "The child understands the logic of classes and relations and can coordinate series and part-whole relationships dealing with concrete things" (Spencer, 1971, p. 208).

4) Formal operational (11-adulthood) - "Characterized by the logic of propositions, the ability to reason from a hypothesis to all its conclusions, however theoretical. This involves second order operations or thinking about thoughts or theories rather than concrete realities [alone]" (Spencer, 1971, p. 208). 
Next, the second cognitive process involved in constructivism that Piaget referred to as equilibrium (Fosnot, 1996) will be explained. Piaget defined equilibrium as a dynamic process of self-regulation of intrinsic behavior, balancing two poles of behavior: assimilation and accommodation (Fosnot, 1996).

Piaget proposed the concept of biological equilibrium as a learning mechanism related to a human being's abilities to adapt to stimuli. He described this adaptation process as being one that involves assimilation and accommodation. Assimilation is how a person perceives a new concept depending on his or her prior experiences, interests and way of thinking (Fosnot, 1996). Accommodation is the fusion of reflective and inclusive behavior that allow us to change our perceptions in response to new inputs and explain new concepts so we can function in equilibrium within the context of cognitive learning (Fosnot, 1996). In other words, accommodation involves finding ways to adjust ones' thinking in response to new and changing environmental conditions such that pre-existing behavior patterns are modified or adapted.

\section{Analysis of the problem.}

Piaget's theory of development of human intelligence can be related to world language learning because the theory refers to how people obtain knowledge. As explained above, Piaget's theory refers to stages of learning; sensory motor, preoperational, concrete and formal operational. Typically, American secondary publicschool students start taking world language classes when they are in the last stage of cognitive development as defined by Piaget, the formal operational stage. This is the 
period in which world language students have to use their reasoning skills to understand things that they may never have experienced before.

For instance, in English, there are only two ways to conjugate a verb; the first, second and third plural person (i.e. I, you, we, and they work) and the third singular person (i.e. he/she/it works). In Spanish, there are six different ways, one for each personal pronoun group. This is an example of a difficult concept for a language learner to grasp that is easier to understand when provided with opportunities to use oral language skills for practical conversation prior to the formal operational stage of developmental.

\section{Critique of Jean Piaget's Social Constructivism.}

Jean Piaget's theory is central to the constructivism construct. Piaget (1970) laid the foundation to understand cognitive development as a learning process, as opposed to the traditional view of learning as an immediate effect of one-way transmission of knowledge from the teacher to the students. For Piaget, a key assumption of his theoretical stance is that cognitive development happens internally in the mind of individuals in stages that he refers to as developmental stages.

So, how may a constructivist framework help American students to build world language knowledge and skills necessary to achieve fluency of a foreign language? For Mohr-Schroeder, Jackson, Miller, Walcott, Little, Speler, and Schroeder (2014), knowledge is obtained through social interaction in real settings influenced by the activities, interaction, and participation of the learner. In keeping with this perspective, I argue that all students are capable of acquiring the necessary skills to speak a world 
language and that to achieve a high level of proficiency, students need to be immersed in the language through social interactions that demand that students think and communicate with others in the target language.

Again, Piaget (1970) asserted that learning is constructed when performing actions in the environment and interacting with new knowledge to build upon previous experiences. Similarly, Martínez (2001) argued that the process of language learning is the product of active participation that takes place within situated environments. Students bring to class a series of personal experiences including those that took place during the period of cognitive development that occurred as they learned their first language (Granell \& Salvador, 1994). Those experiences inform how people think about learning another language.

\section{Vygotsky's Description of Social Constructivism.}

The primary question that intrigued Vygotsky (1978) was: What moves the child's spontaneous interpretation of concepts to scientific concepts? Vygotsky pointed out that learners go through a socially determined process that he called the zone of proximal development as they learn (Frawley, 1997). This theory refers to the degree of independent thinking a person is able to do as they gain foundational skills and concepts from a guide, peer-to-peer interactions, and interactions with the subject matter. The individual learns with others' help; we learn through socio-cultural environmental interactions and these interactions are different for everyone. Fosnot (1996) argued that the way humans experience the zone of proximal development varies from person-toperson depending on each subject's ability to understand the logic of scientific concepts. 
Vygotsky indicated that scientific and spontaneous concepts are closely related (Fosnot, 1996). The spontaneous interpretation concept states that one develops naturally as they construct knowledge in the process of sense-making as a result of the way they interpret and assimilate perceptions "emerging from the child's own reflections on every day experience. The scientific concepts are originated in the structured activity of classroom instruction and impose on the child more abstractions and more logically defined concepts than those constructed spontaneously" (Fosnot, 1996, p. 18). The development of spontaneous concepts must reach a certain level so that the child can absorb and use their framework of prior knowledge to interpret a related scientific concept (Fosnot, 1996).

The zone of proximal development concept is a tool that allows us to understand the process of student development. Using this construct, we can examine not only what learning has already been obtained, we can identify a learner's position in the process of maturation. Thus, the zone of proximal development allows us to refine instruction based upon the student's logical patterns of their learning progression as they become increasingly independent thinkers.

\section{Analysis of the problem through the lens of Vygotsky's Interpretation of Social}

Constructivism. How can Vygotsky's theory of cognitive development be related to world language instruction of American secondary public-school students? It is helpful to consider the aspect of his theory that focuses on the role of culture in the development of higher-level cognitive functions. World language instruction starts from learning vocabulary and progresses to building sentences and then to conceptualizing ideas and messages. This learning process happens over time as the teacher and students interact 
with the language and each other in a learning environment.

Some world language concepts are easier to understand and incorporate into one's own body of knowledge than others. On one hand a learning task may be easy to perform when the world language and the learner's native language have similar sentence structures (Granell \& Salvador, 1994). On the other hand, the same task will be more difficult to achieve when the structures of the native and novel language are different.

When the language structures are different, the student will need more time, support, and reinforcement from another person to move through their own zone of proximal development to be able to negotiate a learning task independently when similar linguistic challenges arise. Moreover, social interaction is beneficial to learning when students are exposed to real situations wherein, they must apply new knowledge to communicate clearly and receive immediate feedback (Granell \& Salvador, 1994).

\section{Critique of Social Constructivism.}

Again, Vygotsky (1978) stated that learning is a condition of cognitive development that requires the assistance of others and is phenomena that occurs within a zone of proximal development that is different for each individual learner. In other words, for Vygotsky (1978), the individual requires social contact with others to build learning although each person learns at their own pace.

For Rogoff (2003) the construction of knowledge is not a linear process, but instead involves constant forward and backward movements. Similarly, Granell and Salvador (1994) argued that knowledge construction is a process of restructuring and rebuilding in which all new knowledge is generated and builds upon the foundation of previous 
perceptions.

Based on the constructivist perspective, new learning depends on learner's constant interactions with activities, the language, and other people (Martinez, 2001). It is these personal experiences that allow students to transfer knowledge of how to learn language from their memory to construct meaning as they learn a world language.

Indeed, to create optimal learning experiences for their students, teachers must operate as full members of communities of practice (Lave and Wenger, 1991) with their students and continue to learn. To do so, constructivist theory assumes that all teachers should be active consumers of research and develop their own reflective practices to determine how to adapt their teaching practices to better suit the requirements of their students depending on the specific needs of the learners at their particular position within a zone of proximal development (Vygotsky, 1978).

\section{Situated Learning Theory}

Equally important as Social Constructivism to this study is the theory of Situated Learning. Stein (1998) described that Situated learning involves four important principles; 1) Learning is based on everyday actions. This means that teachers and students share their thoughts back and forth in a setting where the action or activity makes sense; 2) Knowledge is obtained intentionally and applied to similar situations. When learners clearly understand the utility of a concept, skills, or in this case, word or language rule, they become empowered to apply the same ideas to other, similar situations; 3) Learning is the result of socialization. Situated learning is a powerful approach to consciously creating teachable moments as co-learners that provide students 
with opportunities to grasp new concepts and embed these concepts within their body of knowledge in meaningful ways. Finally, 4) Learning is real; students need to be told the authentic purposes of learning to be motivated to use the target language to negotiate meaning in practical ways.

Arnseth (2008) stated that learning is developed through interaction within an environment, in specific contexts. To achieve high levels of proficiency in a world language, learners need to perform and adapt to stimuli in different authentic scenarios that require students to quickly problem solve how to adapt their use of language to suit specific contexts.

In fact, researchers Cope, Cuthbertson, and Stoddart (2000) argued that the key element to achieve particular skills is to practice the use of language in authentic settings. For example, in immersion language programs, the setting supports students to develop understandings of how language is used during various practical interactions such as during food preparation, while playing games, and within the context of other common interactions that require rapid adaptations to oral communication.

Within situated learning contexts, attention is placed on the development of higher order thinking where simple memorization and recitation of the content is not as important as providing opportunities for students to fluently apply content knowledge to smoothly facilitate common, daily interactions. Cope et al. (2000) stated that the content of lessons should be relevant to students' daily lives so they feel familiar with the learning task. Furthermore, Stein (1998) argued it is beneficially to group students within 
a close age range so that they feel comfortable sharing ideas, thoughts, and points of view.

Fostering a sense of a community of practice (Platt, 2012) provides a comfortable setting for social interactions among students and the instructor to take place as they engage in conversations using the target language to negotiate tasks. To maximize students' ability to extrapolate the use of language skills to other situations, it is beneficially to students that the setting be relevant for the activity. For instance, students' interactions involving the use of language tend to flow more easily in groups or paired conversation when engaged in tasks that require cooperation to complete. Thus, socialization amongst students within the context of completing group tasks is useful for helping students to acquire world language skills in a way that they will understand and be able to apply in similar scenarios beyond the classroom or camp setting (Chastonay, Zesiger, Klohn, Soguel, Mpinga, Vu, \& Bernheim, 2013).

Moreover, according to Cope, Cuthbertson, and Stoddart (2000), in an immersion program, experts can utilize carefully selected strategies in a highly adaptive manner to support learning. One of the characteristics of learning in a practice setting is that experts or coaches can lead and support students through a series of increasingly complex tasks that move students through their zones of proximal development to become increasingly more independent as they negotiate language challenges.

\section{Analysis of the Problem.}

Stein (1998) stated that to situate learning is the ability to place thoughts and actions in random life situations. The key is to expose students to real problem-solving 
opportunities by selecting specific scenarios for them to respond to in an authentic manner, giving students real time feedback, scaffolding peer interactions, and evaluating their learning from a perspective that is situated within the demands of that particular context. The role of the expert or coach is relevant when designing and conducting situated learning given that this person is aware of the nuances of the contextual demands of the situation and can assist learners in understanding the linguistic and cultural nuances implicit to the situation.

For example, world language students need to be able to use the new language they are learning in both formal and informal conversation. In the context of this particular study, this means involving groups of students in scenarios that require the use of both formal and informal language with coaches on hand who can provide immediate feedback to learners that help them to co-construct understanding with one another in response to the specific demands of the situation.

Group environment plays an important role in world language learning because learning involves sociocultural interactions between people to make sense of how to properly use new vocabulary. Indeed "In the situated learning approach, knowledge and skills are learned in the contexts that reflect how knowledge is obtained and applied in everyday situations" (Stein, 1998, p. 1). Students acquire knowledge in different ways but in order to apply their new knowledge, there must be interactions within an environment that will foster the integration of new terms and concepts into a larger body of knowledge or else new information may be easily forgotten. 
Contu and Willmott (2003) added that situated learning is the combination of body language, peer competition, and knowledge management in a specific environment. For instance, contact with other people with a similar language background tends to be useful for effective integration of new knowledge into one's existing body of knowledge. Groups of students with similar interests and language background contribute to each other's learning because they mutually support each other in a myriad of ways such as providing non-threatening feedback to each other with a shared goal of achieving higher levels of language proficiency and accessing higher levels of learning.

Learning a world language requires more than simply learning oral communication skills. To be able to properly adapt to various types of interactions and settings, students benefit from situated learning experiences that help them to better understand the demands of different culture contexts to properly interpret signals such as body language and particular word choices.

Furthermore, "Situated learning theory encourages a focus not just upon cultural and organizational artifacts, but on the embeddedness of learning practices in power relations, rather than the cognitive contents of individuals' minds" (Contu \& Willmott, 2003, p. 283). For these reasons, the relationships students can build in a conversational language learning environment that involves specific attention to the nuances of how to use languages in adaptive ways result in more powerful world language experiences and higher levels of achievement than is possible in isolated individual learning experiences. 


\section{Critique of the Situated Learning Framework}

Situated Learning is a student-centered theory. In one hand, Cope et al. (2000) claimed that Situated Learning theory assumes that people learn better when they are socially interacting because of people's natural social interactive behavior. People are social beings, always talking to negotiate ideas or thoughts. Similarly, Stein (1998) indicated that learning happens when people interact. People need to interact to each other to meet needs or provide support.

On the other hand, Young (1993) viewed situated learning as contextual within a specific type of learning environment. People need to be immersed in certain environments to stimulate the social interaction. Moreover, Arnseth (2008) and Dede (2009) stated that tools or learning artifacts shape students' interactions. This idea assumes that instructors must be highly intentional when selecting artifacts that will be perceived as relevant and assist learners both in terms of sense-making and motivating the interaction (e.g. introducing terms and phrases related to cooking while in a kitchen using the tools of food preparation).

Moreover, Stein (1998) stated that knowledge is obtained when people are able to apply new knowledge in real life situations. The best way to assess if a person is truly moving towards independence while learning a world language is to intentionally look for moments when students are able to apply their new knowledge in real life situation where the environment plays its role.

Furthermore, for Cope, Cuthbertson, and Stoddart (2000), achieving learning is the capacity to move knowledge to authentic or pretend settings. I posit that people learn 
better when they are immersed in social learning environments involving coaching, peerto-peer interactions, and an environment full of learning tools purposely selected to support learning particular skills in a specific order. When students have these elements in place, learning is fun, has meaning, and imbues students with a sense of purpose.

\section{Literature Review of Immersion Programs}

This section will discuss research related to language immersion programs and world language immersion camp. This literature review will present, analyze, and critique literature related to the value of world language academic programs designed to support public middle school students through language immersion experiences. First, I will present the history and objectives of language immersion programs. Next, I will present different types of immersion programs offered to public school students. Finally, I will describe how this proposed study is situated within the scope of existing relevant literature.

\section{History and Objective of Foreign Language Immersion Programs}

World language immersion programs are the most effective way to instruct a world language (Snow, Padilla \& Campbell, 1988; Goldman, 2018). Likewise, when using immersion program instruction "a wide variety of opportunities emerge for developing students' L1 and L2 proficiencies" (Cummins, 2017, p. 104). This instructional model is not new; its beginnings in North American public schools date from more than five decades ago (Thomas, Collier, \& Abbott, 1993). Bialystok, Peets, and Moreno (2014) pointed out that immersion programs started back in 1960 in Canada where immersion programs received enthusiastic attention from Canadian researchers, 
parents, and educators.

In the 1990's attention was focused on the academic content involved in immersion programs that prepare "students for life in an increasingly interdependent world that is ethnically and linguistically diverse" (Thomas, Collier, \& Abbott,1993, p. 170). Moreover, Thomas, Collier, and Abbott (1993) indicated that the immersion program "was strongly pushed by English speaking parents who wanted their children to be bilingual so that they would have more skills to bring to the job market" (p. 170).

Immersion programs in Canada have expanded across the country; this approach is now broadly implemented in all Canadian provinces. Different variations of immersion programs can be found in Canada. The objective of Canadian immersion programs is to teach Anglophone children to learn French differently from the standard 20-to-30 minutes class day direct-instruction approach by immersing students into learning French as early as possible. Therefore, the Canadian public-school system opened immersion education to kindergarten students. French is used as the instructional language up to third grade where English is partially used as the instructional language.

Thomas, Collier, and Abbott (1993) said;

Among the major goals of immersion are additive bilingualism, high level of literacy in two languages, and academic success. Additive bilingualism refers to the acquisition of an additional language at no cost of one's first language. This concept can be best understood by contrasting it with subtractive bilingualism, sometimes experienced by ethnolinguistic minority groups, when through societal pressures or policies, the ethnic language is slowly replaced by national prestigious language (p. 170).

When students add and keep using a world language, cognitive advantages are expected (additive bilingualism). However, when immigrant students' first language is 
replaced by the new country's national language, these students tend to experience cognitive disadvantages. World language immersion camp can assist in bridging this gap for both groups of students.

Met (1993) stated:

...world language immersion program has four major goals; 1) Developing a high level of proficiency in the foreign language; 2) Developing positive attitudes toward those who speak the foreign language and toward their culture(s); 3) Developing English language skills commensurate with expectations for student's age and abilities; 4) Gaining skills and knowledge in the content areas of the curriculum in keeping with stated objectives in these areas (p. 2).

\section{Types of Immersion Programs}

Met (1993) defined world language immersion program "as a method of...instruction in which the regular school curriculum is taught through the medium of the language" (p. 2). The world language is the instructional vehicle to teach content area subjects. The definition of what an immersion program is differs significantly in structure, but certain terms are consistently used to refer to the percentage of time spent using the target language in class.

For Thomas, Collier, and Abbott (1993) and for Met (1993) a total language immersion program refers to an approach wherein all instruction is provided in the students' native language at the beginning of the immersion experience, then the instructor gradually introduces the target foreign language until each language is used for fifty percent of instruction. This process usually last one-to-five years as students move into upper grade levels.

In partial immersion programs fifty percent of academic instruction is provided in each language "from the first year of the program, giving equal instruction time to each 
language throughout students' schooling, or at least throughout elementary school years" (Thomas, et al., 1993, p. 171). The choice of which core subjects employ the use of the target foreign language is made by the individual school district or school. Thomas, Collier, and Abbott (1993) added that the term early immersion is used when the program begins in kindergarten or first grade. They explained that the term delayed immersion refers to when the immersion program begins in four or fifth grade and the term late immersion refers to programs that begin at or after seventh grade.

Importantly, Padilla, Fan, Xu, and Silva (2013) asserted that the best way to assist diverse socioeconomic and ethnolinguistic students is to expose learners to world language instruction as early in life as possible. They claimed that this approach provides students enough time to become highly proficient in both languages.

Thomas et al. (1993) elaborated that language immersion students typically demonstrate high levels of language proficiency after four or five years in the program. They claim that at this point, students who have experienced immersion style instruction demonstrate higher level of academic achievement in the target world language in comparison to students who receive traditional instruction without an immersion experience.

Moreover, Thomas and Collier (1993) claimed that most immersion program students acquire near native listening and reading proficiency skills. Furthermore, later in 2012, Thomas and Collier (2012) indicated that English language learners surpass native English speakers in upper grade levels. 
Thomas and Collier (1993) identified variations of immersion programs have been developed to meet the need of minority language students in the U.S. For example, one model is called "two-way immersion (TWI)". This model is also known as "bilingual”, “dual immersion”, "dual language program (DLP)”, “interlocking”, “developmental bilingual”, or "maintenance bilingual” schools. In TWI or DLP programs, language majority and minority students receive academic instruction in both languages with the main goal of working together, building minority students' selfesteem, and developing positive intergroup relations. Some schools that use the TWI approach have become "magnet schools to assist with integration of students from diverse socioeconomic and ethnolinguistic backgrounds" (p. 171).

\section{Two Way Immersion (TWI) or Dual Language Program (DLP) Models.}

A study conducted by Lindholm-Leary and Block (2010) mentioned that most of the world language immersion programs in the Unites States are Two-Way Immersion (TWI) also known as Dual Language Program (DLP), or Dual Immersion Programs (DIP). Their research found that the most popular languages taught in these programs are Spanish and English, however there are other types of TWI programs in the Unites States such as Chinese-English programs and Japanese-English programs.

In keeping with this finding, Hammer, Jia, and Uchikoshi (2011) found that although the names for the program may vary, dual language programs are defined as an educational approach wherein students are taught in two languages at the same time. They pointed out that although all immersion programs do not necessarily have to begin at an early age to be called a dual language program, they recommended that students' 
exposure to immersion programs occur as early as preschool.

It should be noted that the aim of Dual Language Programs (DLP) is to provide a quality education to students in both the world language and English to promote higher levels of overall academic achievement. However, it is important to clarify that regardless of the age of students, the involvement of the entire community of family, teachers and support staff, and the school environment define the quality of learning (Lindholm-Leary \& Block, 2010).

Researchers Hammer, Jia, and Uchikoshi (2011) and Lindholm-Leary and Hernández (2011) said that students' levels of language proficiency depend on the type of home and school support children receive in learning both target languages. They both noted that parental education levels strongly influence the quality of support students receive at home, which in turn impacts students' levels of language proficiency. The higher a parents' education level is, the more positive parental impacts tend to be on students' foreign language acquisition. That said, it is expected that students attending DLP or TWI academic program will reach a higher proficiency learning level then students enrolled in monolingual programs.

Interestingly, the benefits of immersion programs are not limited to language learning. Lindholm-Leary and Hernández (2011) and Lindholm-Leary and Block (2010) stated that according to their research, students enrolled in Dual Language Programs (DLP) outperform English Language Learners (ELL) and Latino students enrolled in mainstream reading and mathematics courses. 


\section{World Language Immersion Camps.}

Here, I will present language immersion literature specific to camp models. Research conducted by Trujillo (1982) on world language camps concluded that immersion camp learning environments positively impact teaching and learning world language in comparison to the traditional classroom. Björklund and Mård-Miettinen (2011) added that language immersion camps provide a positive approach to learning a language for students who otherwise do not have the opportunity to live in a bilingual environment.

Vines' (1983) research elicited evidence that supported the notion that the term foreign language immersion camp is typically used to describe instruction provided outside regular classroom settings and the normal school day schedule. The main objective of the world language immersion camp is to offer opportunities for students to improve their language skills by communicating in the target foreign language while engaging in experiences that are authentic to the cultures who use the target language (Vines, 1983). The types of activities teachers provide to students in immersion camps often involve recreating authentic cultural experiences through time-intensive activities that also require a considerable amount of set-up time and are therefore difficult to set up and complete in a typical 50-minute class period (Litaker,1990).

Here, this Spanish immersion camp involved the use of a variety of manipulatives to recreate a foreign village setting. The camp provided activities such as cooking, crafts, singing, dancing, shopping, watching movies, and playing games as means to motivate and engage students learning a foreign language. Throughout each activity, the instructor 
played a crucial role by scaffolding the use of language and pronunciation and modeling the use of Spanish to communicate.

Obviously camp instructors play a critical role to the success of immersion camp learning experiences. Next, I will discuss the characteristics and role of Spanish language camp instructors described in literature I read.

\section{World Language Immersion Camp Instructors}

The core classes in language immersion programs must be taught using the target language as the primary communication vehicle, therefore, the success of a world language immersion camp greatly depends on the quality of language instructors. Björklund and Mård-Miettinen (2011) indicated that optimally, language immersion programs ought to be instructed by native or near native speakers of the target language. Likewise, Lang (1993) indicated that a key factor to the success of a language immersion model is to have native foreign language speakers who act as excellent language models and introduce students to the foreign culture through various different activities.

In addition to oral communication, teaching materials also need to be written and introduced in the target language to motivate students to communicate as much as possible in the novel language. Involving native or near-native speakers in the development and implementation of both the written and oral elements of instruction are optimal to ensure accurate and precise communication that follow the rules of the target language (Lang, 1993). 


\section{World Language Immersion Camp Programs and Community}

Platt (2012) explained that a common thread that runs through the philosophical influences of immersion programs is that cultural knowledge is created and passed through human connections. Platt (2012) noted "Immersion, from this perspective, occurs through developing relationships and dialoguing with people within the targeted cultures" (p. 2). World language immersion camp offers students the opportunity to use community resources as part of their language learning process. Vines (1983) supported this notion by saying that immersion language camp is a rewarding experience for teachers as well as for students. Platt (2012) added: "In immersion education, context and the local community play a central role in helping students develop critical consciousness" (p. 5) from a perspective authentically rooted in the cultures of the native speakers of the target language.

For example, organizers may use community resources to provide students with opportunities to engage in conversations with native speakers. In the case of a Spanish immersion camp, recreating restaurants or actually visiting establishments run by native Spanish speakers from historically Spanish speaking cultures creates opportunities for authentic interactions such as discussing traditional cuisines and recipes, placing an order, and engaging in discussions involving the payment process. Exposure to community resources during an immersion camp experience provides opportunities for students to share observations, interpret novel concepts or ways of communicating ideas, and construct new knowledge within a situated learning context (Platt, 2012). 
Furthermore, immersion camps typically involve exposing students to unfamiliar contexts. Unfamiliar contexts such as shopping in a Hispanic outdoor market that appear nothing like a supermarket in the Unites States or cooking unfamiliar dishes using ingredients student have not seen before helps students to compare other people's culture and idiomatic expressions with their own.

"For many it is as if the experience acts as a mirror in which an increasing awareness of one's own self and one's own cultural context takes place" (Platt, 2012, p. 3). In other words, the experience of comparing and contrasting cultures and terminology used in English and Spanish expands students' understanding of phraseology and meaning in novel contexts.

Furthermore, Trujillo (1982) stated that immersion camps are particularly impactful because they tend to be unforgettable experiences for students. This researcher claimed to have shown that fun activities involving play while increasing language skills motivates students to think and interact in a foreign language. He claimed that immersion camps provide students with opportunities to broaden their vocabulary outside the classrooms through interactions with peers in ways that may help students to acquire self-confidence as they become more accustomed to speaking the language they are learning in ungraded, low-stakes situations involving high level motivation to understand and be understood.

Björklund and Mård-Miettinen (2011) argued that the learning community extends to students' families as well. They pointed out that parents play an important role in the success of language immersion programs. According to Björklund and Mård- 
Miettinen (2011) "Parents also understand that once children have learned a new language it is easy for them to acquire additional languages" (p. 23). Therefore, parents with this awareness tend to encourage their children to practice and gain confidence to learn the target language and consider pursuing additional languages as well. These researchers pointed out that trusted adults often serve as key sources of motivation who enforce the use of the target language at home and reward students' progress (Björklund \& Mård-Miettinen, 2011). When children are motivated and offered real opportunities to practice what they have learned in class or extra curriculum academic support, they tend to dare to use their language skills to speak with native speakers in real situations and demonstrate their new skills at home.

\section{Synthesis of the Research Literature}

This section of the literature review will present a synthesis of the literature I read related to language immersion programs in the Unites States. The sub section will begin by describing two cognitive advantages of language immersion camp models.

"Bilingualism can have certain cognitive advantages" (Prevoo, Malda, Mesman, \& van IJzendoorn, 2015, p. 238). The most observable cognitive advantage seen in immersion program students is directly related to language development (Bialystok, Peets, \& Moreno, 2014). Furthermore, Bialystok et al. (2014) emphasized that earlier and deeper exposure to two languages at the same time provides students with opportunities to further literacy skills in both languages.

Interestingly, research has demonstrated that students' experience of constantly switching from using their first language to second language in language immersion 
camp settings may confer particular cognitive advantages (e.g. Platt, 2012; Prevoo, Malda, Mesman, \& van IJzendoorn, 2015; Bialystok, Peets, \& Moreno, 2014; Padilla, Fan, Xu \& Silva, 2013; Snow, Padilla \& Campbell, 1988; Thomas, Collier, \& Abbott, 1993; Met, 1993; Lindholm-Leary and Block, 2010; Hammer, Jia, and Uchikoshi, 2011; Lindholm-Leary and Hernández, 2011; Trujillo, 1982). For example, a study conducted by Prevo et al. (2015) showed that bilingual children usually perform better than their monolingual peers "on executive control tasks, working memory, metalinguistic awareness, abstract and symbolic representation skills, and spatial perspective taking," (p. 238).

In light of excellent results of immersion programs found within the Canadian system of education, a slowly growing number of educators in the Unites States have decided to incorporate immersion programs in American education (Bialystok, Peets, \& Moreno, 2014). In particular, Padilla et al. (2013) indicated that two-way immersion programs have become increasingly popular in elementary school in the Unites States in the last two decades.

Moreover, Padilla et al. (2013) added that two-way immersion program students achieve as high as or higher than their non-immersion classmates in California statewide tests in core subjects (i.e. English language arts, math, and science). These results indicate that when students are immersed in bilingual education, they not only emerge as bilingual but many also demonstrate higher academic proficiency in other disciplines then their counterparts who do not gain access to this support.

That being said, there are significant inconsistencies in the way that world 
language immersion programs are being implemented in the Unites States. For instance, there are many cases where immersion programs are being confined to elementary level only and primarily used as a vehicle to instruct immigrant students until they can be mainstreamed in regular courses taught entirely in English (Maltais, 2012).

As previously mentioned, immersion programs have been shown to be most effective when offered over a period of at least five-to-six years (Gardner, 1983). Thus, limiting immersion experiences to the primary years has been shown to limit the cognitive benefits for students (Wallis \& Steptoe, 2006; Rubinstein-Avila, 2017; and Valdés, 1995).

Despite the wide variety of languages spoken by people in the Unites States, Spanish-English is the most popular world language offered in Two-Way-Immersion (TWI) in elementary public schools in the Unites States (Padilla, et al., 2013). This emphasis on Spanish-English TWI programs may be due to rapidly increasing Hispanic populations in the Unites States. Consequently, the majority of the research on immersion programs has been focused on programs that involve teaching and learning Spanish.

Although educators in the Unites States learned a great deal from Canadian language immersion models, there are important differences in the ways in which immersion programs are being implemented in the two nations. Take for instance the elementary level language immersion program models. While the Canadian elementary level immersion models are designed to teach French to Anglophone students as a second language (Bialystok, Peets \& Moreno, 2014), the Unites States elementary educational 
system largely utilizes immersion programs to support integration of immigrant students into schools by teaching academic content subjects in their native language while they are in the process of becoming proficient in the English language (Padilla, Fan, Xu \& Silva, 2013). After helping students adapt to learning in English, opportunities to access world languages immersion tend to cease (Wilson, \& Kamanā, 2011). Beyond immigrant groups, there are relatively few elementary students receiving TWI language instruction in the Unites States; again, the majority of world language instruction takes place through direct instruction elective courses offered at the middle and high school levels.

Maltais (2012) pointed out that the low number of immersion programs found in this nation is clearly insufficient to meet the needs of our population. To illustrate, Maltais (2012) found that there were 502 language immersion programs in the Unites States in 2001. Of that number; 242 were one-way immersion programs and 260 were dual language programs. Ten different languages were taught across all 502 elementary school programs. Maltais (2012) noted that this meant that very few students were “...exposed to foreign language learning from an early age" (p. 6) and fewer still secondary level students had access to immersion programs in the Unites States.

\section{Critique of the Research Literature}

First, I will direct attention to the methods employed in prior research on the topic of language immersion programs in the Unites States. Although researchers such as Cammarata and Tedick (2012) and Gebauer, Zaunbauer, and Mölle, (2013) used quantitative methods for their studies, the majority of the research I found relevant to the topic are qualitative studies. Indeed, this pattern is in keeping with Pufahl and Rhodes 
(2011) recommendation that researchers employ quantitative methods to examine how students' oral communication improves within the context of language immersion experiences. A qualitative study, for a language immersion study, is relevant due to its ability to provide extensively detailed histories of how students progresses with their language learning.

For example, according to Baxter and Jack (2008) qualitative method[s] permit the researcher to have a better understanding of individuals using different evaluations, relationships, and programs. When qualitative methods are used properly, they serve as effective means to inform the development of theories, evaluate programs, and develop appropriate interventions.

Moreover, MacDonald (2012) indicated that the main objective of qualitative method is to interpret and document the individual's growth and viewpoint. Qualitative methods incorporate techniques that allow the researcher to observe the individuals and gather data to analyze and interpret their personalities, patterns, qualities, and behaviors.

Prior studies have largely focused on investigating whether or not instructional methods typically used in immersions program produce cognitive advantages. In fact, as indicated above, the literature I read such as a study conducted by Thomas, Collier, and Abbott (1993) did confirm that there are a number of cognitive advantages to immersion style instruction verses the didactic approach.

While I did find a number of studies that described the ways language immersion instructional methods are effective from a teaching perspective, the study I conducted focused on using a situated learning lens to examine why and how particular immersion 
program instructional strategies are effective (or not) from a student-centered perspective. This dissertation research built upon prior qualitative research to aid understanding of specific forms of support that should be implemented in public schools by describing second year Spanish middle school level students' responses to particular instructional strategies and activities.

Next, I will discuss various names used to refer to immersion programs and potential misconceptions the variety of labels may cause. Currently in the Unites States, as mentioned above, a variety of names have been assigned to world language immersion programs. For example, Two Way Immersion, Dual Language Immersion, Bilingual Immersion, Developmental Bilingual Immersion, and Interlock Immersion are all names used by programs in the Unites States. These names fail to indicate which of two different approaches the programs take, total or partial language immersion (Snow, Padilla \& Campbell, 1988), thus creating confusion when educators are attempting to draw on lessons from other immersion programs to apply in their own setting in a manner that intentionally scaffolds learning experiences in a logical way.

It is conceivable that each different name given to an immersion program may imply slightly different fine-grained educational goals that require different instructional strategies and activities. The overall lack of clear descriptions and categorization may create misunderstandings for parents, educators, and administrative school personnel about the goals of each particular world language immersion program model and make it difficult to understand the implications of research findings on programs that may or may not match the total or partial immersion approach examined in published studies. 
For example, some immersion models are aimed towards supporting students who speak English as a second language to integrate into courses taught in English while others are geared towards supporting native English speakers to achieve conversational level proficiency in a foreign language. Such distinctions matter when analyzing why and how, if at all, particular activities support the development of specific language proficiencies. Therefore, it is critical that researchers describe whether the programs they study are total or partial immersion and provide not only fine-grain specific goals of curricula, but also characterize how students perceive and respond to particular teaching materials within each context created to fulfill specific instructional goals.

Finally, there is skepticism among parents and school staff regarding how well students learn core content when instruction is provided in a foreign language. "Not all parents or school personnel buy into the concept that students can learn just as much in a foreign language as in their own (Met, 1993, p. 3)”. Furthermore, currently there is no broad consensus among parents and educators on the effectiveness of one language immersion program model over another approach to immersion. Indeed, there appears to be some evidence that warrants confusion.

Met (1993) found inconsistencies in how world language immersion programs are delivered and received by students which led Met to conclude that all models of total world language immersion programs are not for everyone. A limitation that Met (1993) discovered is that total immersion programs require teachers who are highly proficient at teaching core content in English and in the target foreign language for each immersion 
class. Shortages of highly qualified bilingual teachers sometimes force school administrators to hire less qualified teachers in the content area but bilingual.

A practical solution that some schools have found to solve the problem of a shortage of highly qualified bilingual teachers is to implement partial immersion programs rather than total language immersion programs. Partial immersion programs require fewer world language teachers because one world language teacher can spend half a day in one class and the other half of the day in another class. Thus, one teacher can instruct two different language immersion classes in a single day. Therefore, partial immersion programs are easier to manage and the potential effects on staffing is minimized.

This solution comes across as a more feasible alternative for parents for a number of reasons, one of which is that some are uncomfortable with the idea of asking their students to learn to read in a language other than English all day, every day. In fact, Met (1993) claimed that the partial immersion approach seems to be more acceptable to a wider range of parents and school employees.

Unfortunately, there are examples of research that have shown that partial world language immersion program are not nearly as effective as total immersion world language programs (e.g. Met, 1993). Met (1993) stated that students do not develop the same world language proficiency level as students participating in total immersion programs. Met (1993) noted: "A consequence of this is that students may have greater difficulty dealing with the school curriculum in subjects characterized by verbal abstractions" (p. 4). 
In addition, Met (1993) indicated that students enrolled in partial world language immersion program do not achieve the same high levels of English language proficiency in comparison to students enrolled in total immersion world language programs. I argue that there is inadequate research to support this claim and therefore this cannot be a general assumption applied to all partial immersion programs or to all learners. I believe that student learning outcomes in response to partial immersion models greatly depend on the level of student motivation and the forms of support students receive from teachers, parents, and their greater community.

\section{Summary}

In chapter two I presented the theoretical frameworks used in this study and a literature review that describes what is currently known in the field, what gaps exist, and how this study addresses those gaps. In the theoretical framework section, I described rationale for using the Social Constructivist and Situated Learning theoretical lenses to examine students' perceptions within the context of a middle school level Weekend Spanish Immersion Camp held over the course of four-weekends at the same school students normally attend during the week. I presented literature related to immersion camps such as the qualifications of staff that should run a language immersion camp to provide practical opportunities for learners to think and communicate in the target language and ways that the learning environment effect students' knowledge construction by building on existing skills within a situated group learning context.

The literature review section of the chapter briefly described the history and objectives of language immersion programs. This sub section also presented research 
that characterizes language immersion programs, provided different names assigned to immersion programs, and explained confusion these various labels cause for parents, educators, and school administrative personnel.

Finally, I briefly described the idea that the majority of studies conducted heretofore have analyzed language immersion programs from a teacher-centered perspective focused on particular elements of cognitive development. This chapter described the idea that this study addressed a gap in the literature by applying a situated learning lens to examine second year, middle school level Spanish students ' perceptions in response to carefully described instructional strategies and activities that were intentionally scaffolded in a Spanish immersion camp specifically designed to supplement formal instruction.

Next, chapter three will present the research methods I used to elicit and examine student perceptions in the Spanish immersion camp. I will describe my rationale for using a qualitative approach in the study. I will also present the data collection procedures, the data analysis and interpretation plan and the timeline for completion of each major step in the research process. 


\section{CHAPTER THREE}

\section{METHODS}

Chapter three will present the methods used in this study. This chapter will describe the research approach and the rationale for the methods used in this study. The chapter will introduce the research questions and provide an overview of the motivation for examining these particular questions. Next is a description of the context of the study followed by the study participant selection criteria and recruitment process. I will describe the instruments and the data analysis approach used in this study. What follows is the data management plans, potential threats to validity, and controls for those threats. Finally, I will provide a timeline of activities, a description of my positionality as a researcher, and conclude with a brief chapter summary.

\section{Research Methods}

I employed qualitative methods using a participatory observer action research approach. I designed methods to elicit students' perceptions and responses within the context of a supplemental Spanish language instruction program offered in the form of a four-weekend immersion camp.

I chose this approach for this study because "Qualitative method[s] allows the researcher to study individuals or organizations through complex interventions, relationships, communities or programs" (Baxter \& Jack, 2008, p. 544). When qualitative methods are properly employed, they serve as effective means to inform the development of theories, evaluate programs, and develop appropriate interventions. 
Qualitative research incorporates techniques and methods that allow the researcher to observe, gather information to analyze and interpret the personalities, patterns, qualities, and behavior of people. MacDonald (2012) indicated that conducting qualitative research through action research allow the gathering and analysis of data to create knowledge with the main goal of informing action.

McKernan (2013) said:

The rationale for action research rest, initially, on three pillars; first that naturalistic settings are best studied and researched by those students experiencing the problem; second that behavior is highly influenced by the naturalistic surroundings in which it occurs: and third, that qualitative methodologies are perhaps best suited for researching naturalistic settings. (p. 5)

Furthermore, McKernan (2013) argued that action research involves the participation of the researcher and community members who are seeking to improve a situation. Action research discourse can be approached from a myriad of perspectives such as: participatory action research, participatory research, community-based participatory research, and other forms of participative inquiry.

From an epistemological perspective, the nature of Participatory Observer Action Research is such that the researcher and students are assumed to interact. In this study, data that informs the research questions was elicited through students' interactions with the researcher, other camp instructors, their peers, the language itself, and instructional activities involving experiences designed to support reflection. As the Camp Director, designer, and an instructor, I deemed Participatory Observer Action Research an appropriate approach for this study. 
According to Coghlan and Brannick (2014) "much of what we refer to as qualitative research is focused on the past. Action research builds on the past and takes place in the present, with a view to shaping the future" (p. 9). Coghlan and Brannick (2014) developed an Action Research Cycle framework composed of a pre-step; constructing a sense of context and purpose and four basics steps; constructing, planning action, taking action, and evaluating action. Figure 1 illustrates this framework.

\section{Figure 1}

\section{The Action Research Cycle}

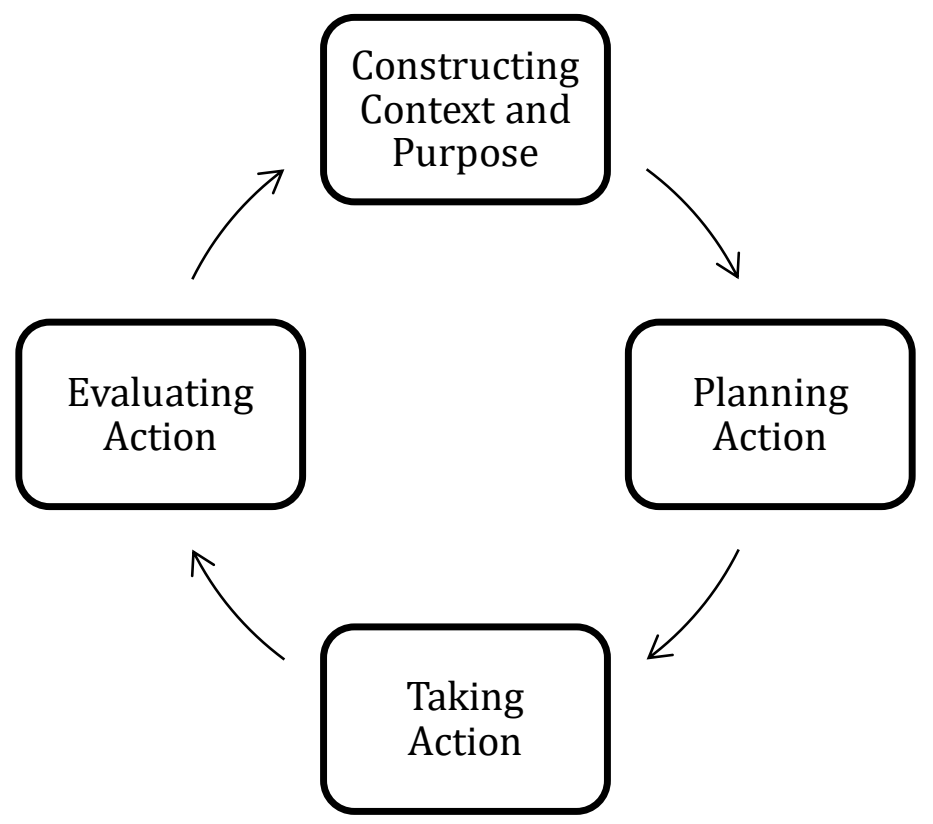

Note. Adapted from Doing action research in your own organization by Coghlan, D., \& Brannick, T. (2014) Sage.

The process starts with understanding why the project is important and necessary to invest time to study. Assessment of the internal and external context of the issue gives clear direction for how to approach analysis of social phenomena. The external context 
could be the economic, political, and social forces that are driving change. Examples of influential internal forces are the cultural context and structure of the issue.

After establishing clear understanding of why, if at all, a particular social phenomenon is worthy of examining, constructing knowledge within an action research cycle involves dialogic activity to understand what the key influential issues are and whether or not sufficient evidence exists to suggest that a topic or issue is ripe for further study (Coghlan \& Brannick, 2014). Coghlan and Brannick (2014) described this step as a collaborative, constructive venture wherein the researcher engages with others to make this determination. A key purpose of this step is to define the research problem.

Based on the review of the literature and personal experiences as a veteran language teacher working in community with other middle school level teachers, I determined that the problem that this study would address is understanding how middle school students perceive that immersion camp experiences effects their own language skills development. I posed three open-ended research questions:

1. To what extent do middle school Spanish Two students perceive that Spanish immersion camp instruction serves to assist retention of world language communicative skills?

2. What kind of activities from the Spanish immersion camp effectively support Middle School students learning world language?

3. How does the Spanish immersion camp environment influence Middle School students' perspective of their own world language learning process? 
The study was motivated by the belief found within my educational community that learning languages is a process that, by its very nature, requires interaction with other people and is based on interactions within the environment. Rather than simply using memorized words, conversational fluency requires that a person adaptively co-construct knowledge and skills using the target language to negotiate everyday situations that deals with their social environment.

Martin and Loomis (2013) supported these ideas by saying that people learn best when constructing their own knowledge and their understanding of any situation. Thus, creating a learning situation designed to support naturalistic interactions amongst learners and examining their responses throughout language immersion experiences became a key source of motivation for this study.

Specifically, the aim of this study was to create a thick, rich description of students' responses to the structure of activities in terms of their learning and their perceptions of how their experiences did or did not assist with their Spanish language retention and development. Another key objective was to draw on student feedback to identify ideas for better ways to scaffold and support students' world language learning through a language immersion camp. Special attention was paid to students' language barriers, emotional responses, language skill development, and social interactions as they were using Spanish to communicate.

The next sub section provides an overview of the context of the study. I begin with a description of the setting where the camp was held followed by a brief outline of activities involved in the camp. 


\section{Setting}

The context of this study was a four-Weekend Spanish Immersion Camp held at the middle school where I teach and the study students attend middle school. The study was conducted from October 27th to November 17th 2018. At this point of the academic year my students had received eight weeks of year two Spanish instruction. The camp was held from Friday at 4:00 PM until Saturday 6:00 PM on each of the four weekends. Activities were held in the school auditorium, four classrooms, the library, the gym, and the staff lunch room that has a small kitchenette with a refrigerator and stove.

\section{Description of the Spanish Immersion Camp}

The camp involved three types of immersion activities designed to support students use and development of Spanish language knowledge and skills. These activities involve learning, physical, and cultural activities.

\section{Learning Activities.}

Students engaged in group language learning sessions facilitated by language instructors who speak Spanish as their native language. There was one instructor per every three students. The purpose of these sessions was to refresh linguistic concepts already learned in their formal Spanish Two classes and to provide students opportunities to implement these concepts through activities designed to encourage group interactions wherein students were asked to communicate only in Spanish.

\section{Physical activities.}

The camp involved physical activities, such as games, designed to stimulate body movement. The purpose of these games was to encourage students to literally think on 
their feet by challenging them to use Spanish to give and receive game instructions, to play the games, and provide peer support.

\section{Cultural Activities.}

The cultural activities took place in the gym, classrooms, and staff lunch room. Students participated in salsa dancing, made Hispanic art, and learned to cook Latin cuisines.

The salsa dancing was facilitated by a salsa instructor. She was specifically hired to teach this activity using only Spanish to guide the dancers in a large, open corridor.

The camp meals were prepared and consumed by the students and chef in the staff lunch room kitchenette. Students received Spanish instruction while cooking traditional Hispanic dishes from a variety of Latin cultures. A Latino chef worked directly with students to explain preparation of various Hispanic cuisines and describe the cultural roots of the dishes in Spanish over the course of the four weekends. Students acted as chef helpers; they were required to communicate with each other and the chef entirely in Spanish.

The artistic segments of the camp were conducted in a classroom. Activities consisted of making traditional crafts from various Hispanic cultures such as making piñatas and party decorations for the last day of the camp.

At the end of the camp, the students and instructors hosted a party for the parents to showcase what was learned from the dance lessons, cooking experiences, language development activities, and art lessons experienced during the four-weekend Spanish immersion camp. On the final day of the camp, after student presentations, the students 
hosted a final meal wherein camp participant explained to the audience in Spanish how they made the food that represents different Latin cultures.

\section{Participants}

\section{Staff.}

The camp was led by the researcher who is Middle School teacher of students. The camp needed language instructors to support students. These language instructors needed to have some experience on school or educational support. One language instructor was also music instructor. The camp needed a chef for cooking sessions. The chef needed to be Hispanic cuisine knowledgeable. We also hired a professional salsa instructor. She is in the dance business for 15 years. All staff were instructed on the main objective of the camp; immerse students into the language.

The following table displays some of the characteristics and background of the staff who took part on this research. The main objective on hiring these staff was to provide Spanish language support during the camp immersion.

Table 3

Weekend Spanish Immersion Camp staff

\begin{tabular}{|l|l|l|l|l|l|l|}
\hline Name & Age & Sex & Ethnicity & L 1 & L 2 & Background \\
\hline $\begin{array}{l}\text { Researcher } \\
\text { and Spanish } \\
\text { teacher }\end{array}$ & 57 & Male & Hispanic & Spanish & English & $\begin{array}{l}\text { Spanish teacher at } \\
\text { the Middle school, } \\
\text { native Spanish } \\
\text { speaker. }\end{array}$ \\
\hline $\begin{array}{l}\text { Language } \\
\text { instructor 1 }\end{array}$ & 23 & Female & Hispanic & Spanish & English & $\begin{array}{l}\text { School tutor, Big } \\
\text { brothers and Big } \\
\text { sisters volunteer } \\
\text { program, native } \\
\text { Spanish speaker. }\end{array}$ \\
\hline $\begin{array}{l}\text { Language } \\
\text { instructor 2 }\end{array}$ & 22 & Female & Hispanic & Spanish & English & $\begin{array}{l}\text { School tutor, Big } \\
\text { brothers and Big }\end{array}$ \\
\hline
\end{tabular}




\begin{tabular}{|l|l|l|l|l|l|l|}
\hline & & & & & & $\begin{array}{l}\text { sisters volunteer } \\
\text { program, music } \\
\text { instructor, native } \\
\text { Spanish speaker. }\end{array}$ \\
\hline $\begin{array}{l}\text { Language } \\
\text { instructor 3 }\end{array}$ & 29 & Male & Hispanic & Spanish & English & $\begin{array}{l}\text { Mental Health } \\
\text { Specialist, native } \\
\text { Spanish speaker. }\end{array}$ \\
\hline Chef & 45 & Female & Hispanic & Spanish & English & $\begin{array}{l}\text { Hispanic Cuisine } \\
\text { Chef, native } \\
\text { Spanish speaker. }\end{array}$ \\
\hline $\begin{array}{l}\text { Dance } \\
\text { instructor }\end{array}$ & 34 & Female & Hispanic & Spanish & English & $\begin{array}{l}\text { Professional Salsa } \\
\text { Instructor, native } \\
\text { Spanish speaker. }\end{array}$ \\
\hline
\end{tabular}

\section{Students.}

The study and camp participants were students from a middle school located in the Pacific Northwest region of the Unites States that hereafter will be known as the SIC middle school, a pseudonym assigned to protect the privacy of the students. All students were eighth grade students enrolled in the second year of a Spanish world language program in the same school where this study and camp took place over the course of four weekends. All students were enrolled in the same Spanish Two class together.

The typical class size of Spanish Two courses in the middle school where this study took place is between twenty-to-thirty students. Therefore, the plan was to recruit between fifteen-to-twenty-five middle school level students to participate in the camp and this study. In the end, nine students were actually recruited to participate. 
Table 4

Weekend Spanish Immersion Camp students

\begin{tabular}{|c|c|c|c|c|c|c|}
\hline Name & Age & Sex & Ethnicity & L 1 & L 2 & Background \\
\hline Carlos & 13 & Male & American & English & & $\begin{array}{l}\text { 8th grade Middle } \\
\text { School student, } \\
\text { native English } \\
\text { speaker. }\end{array}$ \\
\hline Leah & 14 & Female & American & English & & $\begin{array}{l}\text { 8th grade Middle } \\
\text { School student, } \\
\text { native English } \\
\text { speaker. }\end{array}$ \\
\hline Lorita & 13 & Female & American & English & & $\begin{array}{l}\text { 8th grade Middle } \\
\text { School student, } \\
\text { native English } \\
\text { speaker. }\end{array}$ \\
\hline Brianna & 13 & Female & American & English & & $\begin{array}{l}\text { 8th grade Middle } \\
\text { School student, } \\
\text { native English } \\
\text { speaker. }\end{array}$ \\
\hline John & 14 & Male & American & English & & $\begin{array}{l}\text { 8th grade Middle } \\
\text { School student, } \\
\text { native English } \\
\text { speaker. }\end{array}$ \\
\hline Isabella & 14 & Female & American & English & & $\begin{array}{l}8 \text { th grade Middle } \\
\text { School student, } \\
\text { native English } \\
\text { speaker. }\end{array}$ \\
\hline Giovanni & 14 & Male & American & English & & $\begin{array}{l}8 \text { th grade Middle } \\
\text { School student, } \\
\text { native English } \\
\text { speaker. }\end{array}$ \\
\hline Juana & 13 & Female & American & English & & $\begin{array}{l}\text { 8th grade Middle } \\
\text { School student, } \\
\text { native English } \\
\text { speaker. }\end{array}$ \\
\hline María & 14 & Female & American & English & & $\begin{array}{l}\text { 8th grade Middle } \\
\text { School student, } \\
\text { native English } \\
\text { speaker. }\end{array}$ \\
\hline
\end{tabular}




\section{Participant recruitment.}

I used a purposive sampling technique to recruit second year Spanish students to this camp from the middle school where I teach. Maxwell (2005) pointed out that there are four main goals for purposeful sampling: 1) Find representation of the context, which includes the setting, the individuals, and the activities to be develop during research;2) Locate heterogeneity in the population to represent the entire range of variations; 3) Evaluate situations that are critical for the theories that the researcher is going to study or develop; and 4) The researcher has to create comparisons to explain the reasons for differences between settings or individuals.

Here, the main reason for recruiting second year Spanish students is that these students had acquired enough vocabulary and understanding of Spanish linguistic conventions to coherently build sentences and orally communicate with each other. Moreover, I posited that these students were going to benefit from this immersion experience because, although they had obtained sufficient Spanish language instruction to hypothetically negotiate oral communication tasks, they lacked access to sustained interactions involving close mentorship to utilize and build on knowledge learned in class.

As previously stated in chapter two, I argue that mentored experiences within the context of a language immersion program strongly influences high levels of achievement. This is because students co-construct knowledge and skills based on immediate demands to use the language as a communication tool rather than engaging in a rote learning experience involving lists of decontextualized words and rules to memorize and recite. 


\section{Participant Selection}

The selection process was based on two criteria. First, students had be officially enrolled in the target middle school level for the 2018-2019 academic year and secondly, students and their parents were required to complete the enrollment package to participate in the camp and the study.

\section{Protection of Human Subjects}

This participatory observer action research followed Human Subjects research policies and procedures mandated by my university and the school district where the study took place. No data was collected prior to approval from both Institutional Review Boards (IRB).

Following IRB approval, students and parent were notified of the opportunity to enroll to the Weekend Spanish Immersion Camp. Interested parents and their children were asked to complete an enrollment form in order to be admitted into the program. The enrollment package contained: a description of the supplemental instruction Spanish immersion camp, learning outcome goals, instructional activities and educational benefits, the data collection procedures, and camper behavioral guidelines (see appendix C).

The enrollment packet also contained an active parental informed consent form, an active student informed assent form, a student enrollment form and a form designed to collect critical students' information (personal information, emergency information, medical insurance information, and medication prescription information).

Finally, the enrollment packet contained a form that requested of potential students that they write and submit short responses to two essay questions described 
below. If a student sensed an unacceptable level of risk or potential harm or any type of discomfort, they were explicitly told in writing and verbally that they may withdraw from the study at any time without this decision impacting their participation in the camp. Furthermore, the researcher guaranteed that students' identity was protected regardless of participation in the study.

\section{Instruments and Measurements}

Qualitative methods provide researchers with the advantage of analyzing phenomena using a variety of lenses to better understand nuances of human perceptions (Creswell and Miller, 2000). For this reason, I selected three instruments that provided the opportunity to triangulate the data. The three qualitative instruments I employed were:

1. A pre-camp short answer response essay. As mentioned above, the enrollment packet included a form that posed two questions with a request that students respond to the items in writing to share their initial insights and expectations of the Weekend Spanish Immersion camp. These questions were, why do you wish to participate in this learning camp and what are you expecting to see and get during this four Weekend Spanish Camp? There was not a word limit for this short-answer response style instrument.

2. Classroom observations; throughout each activity I handwrote observations of students' use of language. Every ten minutes I noted levels of student engagement as high, medium, or low and noted the percentage of students exhibiting on-task behavior. 
3. Post-camp student interviews; conducted as 15-to-20-minute semi openedended interviews in English involving one student at a time. This semi openended interview involved using a small set of prepared questions to stimulate conversation and then allowing for new questions to arise throughout the conversation. Prepared questions were provided to students in advanced to allow them time to consider their responses prior to the discussions. These prepared questions involved the two questions posed in the enrollment packet plus other questions that arose in relation to the three research questions.

Each interview was audio recorded and videotaped, transcribed and coded using a dialogical coding technique. The transcription of all interviews was conducted by one of the language instructors who was specific instructed to transcribe the interview recordings verbatim including all verbal utterances. Each camp student was assigned a pseudonym to ensure protection of respondents' identities.

\section{Data Collection and Analysis}

According to Krathwohl (2009), data and analysis are interdependent with each other because data will determine the direction of the analysis. Here, all three forms of data were analyzed using data coding techniques on an on-going basis as data was collected. For example, analysis of student responses to the essay questions in the enrollment packet during the camp planning phase informed changes to the planned activities, assessment instruments intended to formatively assess student learning, and later, changes made to the interview prompts. 
The dialogical coding strategy described in Thematic Networks Manual by Attride-Stirling (2001) was followed step-by-step to find themes in the enrollment packet student essays, classroom observation notes, and post camp interviews. For example, the post camp interview data was transcribed semi-verbatim and coded using an open coding and focused coding strategy to find themes of student perceptions within the data sets.

The Thematic Networks Manual coding strategy involves three stages; a)

Reduction or breakdown of text; b) Exploration of the text; and c) integration of the exploration. Figure 2 provides a snapshot description of the steps involved in each of these three stages of data analysis.

Figure 2

Analytic Stages Used in this Study

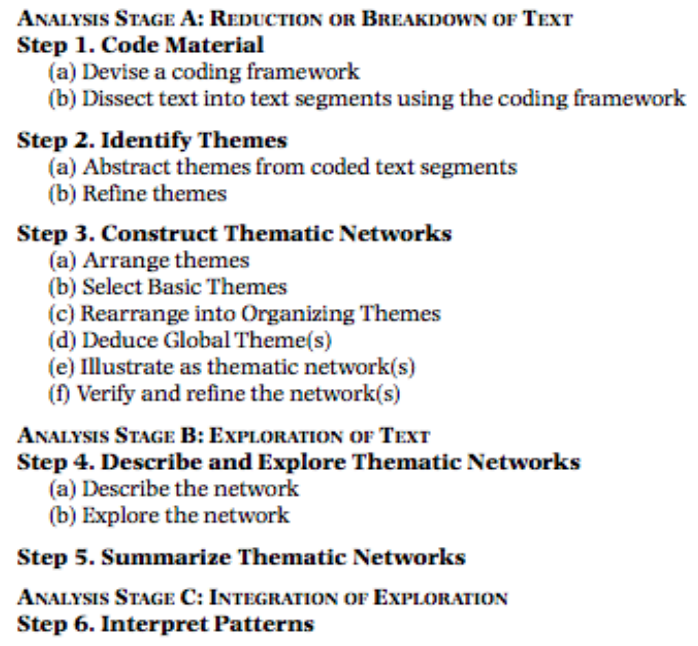

Note. Figure from Thematic Networks: An analytic tool for qualitative research, by

Attride-Stirling, J. (2001), Sage.

\section{Analytical Stages}

My data analysis process was divided into these three stages. While all three 
stages involve interpretation, each stage requires a more abstract level of analysis used to describe the data.

\section{Reduction or Breakdown.}

The first stage of data analysis was divided into three steps; coding material, identify themes, and constructing thematic networks. Here, the student essay responses and post camp interview transcripts were rewritten as sentence strips. Each sentence strip was assigned an initial code that was the first word that came to mind when I read each sentence strip. Then, I reduced the data by organizing the coded strips into groups of initial codes that shared the same or similar meaning and assigned a focus code for that collection of initial codes. Next, I organized these focus codes into clusters and analyzed these codes for themes.

Similarly, the classroom observation notes were assigned initial codes and focus codes. Next, the focus codes were grouped within their own sets of identified themes.

I also created a data table that arranged each activity into groups divided by three categories of students' levels of engagement (high, medium, and low) and achievement to analyze levels of student responses and determine in what ways activities appeared to support students' engagement and their use and development of language knowledge and skills.

\section{Exploration of Text.}

The exploration of text stage was divided into steps four and five. Step four was describing and exploring the networks. This step involved examining the relationships between the themes to establish thematic networks and describing the contents of each 
thematic network with supporting text segments that provided evidence to support the claim that each thematic network was in fact present within the data sets (Attride-Stirling, 2001).

Step five was to summarize the thematic networks. Here, the main ideas and characterizing patterns were recorded. To interpret the data, I examined each of the data sets collected into thematic network clusters with the goal of detecting what, if any, underlying patterns appeared. I then summarized the highlighted patterns found within each of thematic network across each of the data three sources, (student short essay responses, work samples, and interviews).

\section{Integration of Exploration.}

This last stage involved a single step, which was the interpretation of patterns.

Once the thematic networks and patterns that emerged across the data sets were summarized, I determined the implications of the thematic networks as they related to the research questions. I then expressed the implications of interpretation of the data in terms of students' perceptions of the effectiveness of the camp strategies and activities and how these ideas influenced changes to the immersion program structure and activities.

\section{Data Maintenance}

All hard copy data is stored in a locked file cabinet in my home office. Digital data is stored on my personal computer and in a password protected Google Drive folder. Sharing of the raw and analyzed data sets was restricted to the dissertation committee members and fellow camp language instructors. To protect the privacy of students, actual names were not used. Pseudonyms were assigned for the school district, school, camp, and student. These pseudonyms were tracked in a digital code book stored in a 
password protected file on my personal computer that I did not share with anyone and destroyed at the conclusion of the study.

All raw data will be destroyed five years after the final manuscript has been completed and approved by my university Office of Graduate Studies. Hard documents will be cross shredded and electronic data will be permanently deleted from my computer and cloud-based Google Drive file folders.

\section{Role of the Researcher}

I am from Peru and was born in the capital city, Lima. I am the first person in my family to pursue a doctoral degree. While in Peru, I served as a world language teacher where I taught English as foreign language for fifteen years prior to moving to the Unites States to become a Spanish language teacher.

I am a bilingual and bicultural educator. Spanish is my first language; I learned English as a foreign language in my native country. In the Peruvian educational system, every high school student receives two hours of foreign language instruction weekly. English is a mandatory subject that high school students are required to take for five consecutive years. Thus, when I arrived in the Unites States. I thought that I would be able to easily navigate with the English language knowledge I learned in school, but this was not the case.

The basic knowledge I received while in my country was a helpful start, but this foundation did not prove to be sufficient to smoothly navigate all types of social interactions within the Unites States. I have learned through personal experience that a person needs to be immersed in the target language to develop high levels of proficiency. 
I immigrated to the Unites States almost twenty years ago. At first, I was hired as an Educational Assistant while I attended Portland State University in the state of Oregon to earn a Bachelor degree in education. Later, I continued my education and obtained a Master degree in Curriculum and Instruction.

I have been a middle level world language teacher in the Unites States since 2003. I teach Spanish to students who speak English as their first language and also Spanish for Spanish Speakers. In the school district where I teach, secondary public students take world language daily for one hour, from one-to-five years at the most.

As a teacher, I have observed public school students in both Peru and the Unites States where I have found that even after taking world language classes for several years, most of my students do not reach a high level of foreign language proficiency unless they are immersed in the target language and culture. However, it is my belief that all students are capable of achieving high levels of proficiency with the right academic support. With this in mind, I conducted this piece of research to provide a student-centered description of public-school students' perceptions of how language immersion activities contribute to their efforts to reach high levels of world language proficiency.

I was the Spanish teacher of students who were participating in this Weekend Spanish Immersion camp. My role in school was (and is) to instruct Spanish to middle school students Monday to Friday. My role in the Weekend Spanish Immersion camp was to organize the camp, meet with language instructors, observe the students and staff interactions and float around the activities. 


\section{Potential Threats to Validity}

Here, I will discuss potential threats to the validity and trustworthiness of the data. According to Maxwell (2005) validity is a "fairly straightforward, commonsense way to refer to the correctness or credibility of a description, conclusion, explanation, interpretation, or other sort of account" (p. 106). For the purpose of this study I examined students' perceptions from three different perspectives; the perspective of the researcher (researcher reflexivity), the perspective of study participants (member checking) and the perspective of people external to the study (peer debriefing), (Creswell and Miller, 2000).

\section{Researcher Reflexivity}

This perspective is also known as personal reflection. Throughout the research cycle, I kept a journal where I recorded critical self-reflections such as explaining my assumptions, beliefs, and biases that may have influenced this study. After assigning the initial codes, I referred to this list of potential biases and reconsidered the assignment of the initial and then final codes to ensure that each code accurately reflected the students' meaning rather than my own bias or assumptions.

\section{Member Checking}

"With members checking, the validity procedure shifts from the researcher to participants in the study" (Creswell \& Miller, 2000, p. 127). Following the transcription of interviews, I requested that each student approve or make corrections to the transcriptions to ensure that their meaning was accurately conveyed. Students were only 
asked to check the interview transcript that they participated in and did not have access to reading the transcriptions from other camp students.

\section{Peer Debriefing}

According to Creswell and Miller (2000) a peer reviewer is a person other than the researcher who is familiar with the study who reviews and adds their perspective to enhance the quality of the data collected. I executed peer-debriefing in two stages for this study. For the first stage, each evening after camp I typed my classroom observation notes and posted them to a Google folder that I shared with the camp language instructors for their review and feedback. The language instructors added their clarifications and additions in brackets so as distinguish the original notes that I took from their changes to the notes.

As part of the weekly peer-debriefing process, the researcher met with the language instructors as a group to review what was to be learned from the classroom observations related to student perceptions of the activities and levels of student engagement that informed adaptations to the following weekend activities. I brought student work samples from the previous weekend camp to these de-brief sessions so as to provide tangible artifacts of students thinking that provided ideas we responded to as a group.

After the final camp day, I typed up the notes as usual and circulated them via the Google Drive for language instructor feedback, however the final post camp language instructor meeting agenda focused first on what was to be learned from the final 
weekend camp session and next on overall reflections on the classroom observations from all four weekends.

In this final meeting, we had a collection of student work samples from all four weekends available to us to use as reflective devices to ground our interpretations of the classroom notes with further evidence of student responses to the activities. These student work samples were not coded, they simply served as references for our discussion and provided opportunities to double check and correct interpretations of student responses as evidence of their levels of engagement and understanding.

Throughout each of these conversations I collected fieldnotes that became part of the classroom observation data analysis and interpretation process. These reflective fieldnotes were considered a data set to code and also be used to adjust or modify the next camp activities.

The second stage of peer-debriefing was conducted to acquire feedback from language instructors on the interviews coding. I shared the coding from the interviews with the camp instructors because of their first-hand knowledge of how the camp was conducted. Therefore, they could, for a qualitative inquiry conversational coding, verify the correctness of the assignments of initial codes to establish inter-rater reliability (IRR). Once this reliability was established, I had the language instructors review the interview codes and thematic networks for each of the nine data sets and for the patterns found across all nine data sets and if necessary, challenge my assumptions on the analysis of the data. Drawing on this feedback, I then adjusted the initial codes as necessary and revised the thematic networks prior to the fifth step in the analysis which was describing the 
interpretation and implications of the thematic networks and patterns in regards to understanding student perceptions of the structure of the experiences and the activities themselves.

\section{Participatory Observer Action Research Timeline}

Here is the timeline of this study:

\begin{tabular}{|l|l|}
\hline \multicolumn{1}{|c|}{ Actions } & \multicolumn{1}{|c|}{ Expected Timeline } \\
\hline Submission of proposal dissertation & May 2018 \\
Proposal defense & May 2018 \\
IRB approval & June 2018 \\
$\begin{array}{l}\text { Submission of proposed of extra academic } \\
\text { support to world language students to the } \\
\text { school administration. }\end{array}$ & July -August 2018 \\
Sent an invitation to parents of students & August 27th, 2018 \\
enrolled in Spanish Two inviting them to \\
attend a meeting to discuss the possibility of \\
their students' involvement in this \\
supplemental language experience.
\end{tabular}




\begin{tabular}{|c|c|}
\hline Collection of application forms & September 17 th to $21_{\text {st, }} 2018$ \\
\hline $\begin{array}{l}\text { Notification to parents of students enrolled to } \\
\text { the Four-Weekend Spanish Immersion Camp } \\
\text { and hire counselors, dance teacher and chef. }\end{array}$ & October 1 st to 5 th, 2018 \\
\hline $\begin{array}{l}\text { Coordination with school administration to } \\
\text { organize the camp and collect student } \\
\text { enrollment packet short essay responses }\end{array}$ & October 2018 \\
\hline $\begin{array}{l}\text { First session of the Four-Weekend Spanish } \\
\text { Immersion Camp - student work sample } \\
\text { collection and analysis }\end{array}$ & The weekend of October 27th 2018 \\
\hline $\begin{array}{l}\text { Second session of the Four-Weekend Spanish } \\
\text { Immersion Camp - student work sample } \\
\text { collection and analysis }\end{array}$ & $\begin{array}{l}\text { The weekend of November 3rd, } \\
2018\end{array}$ \\
\hline $\begin{array}{l}\text { Third session of the Four-Weekend Spanish } \\
\text { Immersion Camp - student work sample } \\
\text { collection and analysis }\end{array}$ & $\begin{array}{l}\text { The weekend of November } 10 \text { th, } \\
2018\end{array}$ \\
\hline $\begin{array}{l}\text { Fourth session of the Four-Weekend Spanish } \\
\text { Immersion Camp - student work sample } \\
\text { collection and analysis and post camp } \\
\text { interviews }\end{array}$ & $\begin{array}{l}\text { The weekend of November } 17 \mathrm{th} \text {, } \\
2018\end{array}$ \\
\hline $\begin{array}{l}\text { Data analysis and interpretation } \\
\text { Complete the dissertation manuscript, }\end{array}$ & $\begin{array}{l}\text { December } 2018 \text { and June } 2019 \\
\text { July 2019- March } 2020\end{array}$ \\
\hline
\end{tabular}


Dissertation defense and submission to the

April-May 2020

Office of Graduate Studies

\section{Summary}

Chapter three described the purpose of the study and of using a participatory observer action research approach to examine the perceptions and responses of middle school level second year Spanish students involved in a Weekend Spanish language immersion camp specifically designed as a four-weekend supplement to their formal language education course offered in the same school where the camp took place. The Spanish immersion camp is described as well as the learning, physical, and cultural activities that supported students' world language learning.

This chapter also focused attention on the process of students' recruitment and selection as well as their protection under the umbrella of Human subject research policies and procedures mandated by the university and school district. I indicated how the data and students' identity is protected.

The chapter presented the research questions, To what extent do Middle School Spanish students perceive that Spanish immersion camp instruction serves to assist retention of world language communicative skills; What kind of activities from the Spanish immersion camp effectively support Middle School students learning a world language, and How does the Spanish immersion camp environment influence Middle School students' perspective of their world language learning process?

Chapter three presented three research instruments used in the study: pre-camp short essay responses to two questions, classroom observations, and nine post camp 
interviews. I presented the analytical approach using coding techniques described by the Thematic Networks Manual by Attride-Stirling (2001) to find thematic networks and patterns within and across the data sets. Finally, this chapter provided a description of the role and positionality of the researcher and an explanation of the three lenses used to validate the data and the timeline of the participatory observer action research.

In the next section, I will present chapter four. Here, I will present the result of the data analysis and interpretation of data collected throughout the study. Chapter four begins with the background of the study and ends with a brief summary. 


\section{CHAPTER FOUR}

\section{RESULTS}

Chapter four will present the results of this action research study. As described in chapter one, the aim of this study was to examine and describe middle school students' perceptions in response to supplemental instruction designed to improve students' world language oral and written communication skills, and comprehension for fluency. The language immersion camp provided students with the opportunity to develop oral language proficiency by communicating solely in Spanish through a four-weekend camp. This non-traditional, informal world language program was called the Weekend Spanish Immersion Camp.

\section{Background of the Study}

This study took place in a Middle School. The Weekend Spanish Immersion Camp utilized parts of the school where I teach and the students are enrolled. The goal was to situate the camp in a safe and familiar environment the students already associated as a place of learning. This included the use of the gym for games and activities that required a large, open indoor area.

Three classrooms were converted into: a girls' sleeping area, a boys' sleeping area, and a room with several work tables for small group academic lessons. During the day the boys' sleeping area, which had a projector and large white board, was also used as a room for singing, crafting, and literature lessons.

The school kitchen became the location of the cooking lessons where students prepared their meals together, set the dining table, and cleaned up after each other. The 
school's unique honeycomb layout also allowed for the use of a spacious corridor as an area for dance lessons.

Another primary reason for holding the camp on school property was that students would spend a large amount of time away from home. This involved four consecutive weekends each consisting of a total of 26 hours starting from 4:00 pm on Friday to 6:00 pm on Saturday. Adult supervision was provided by the teacher and three language instructors, two males and two females, throughout the duration of the camp. See appendix I for a detailed activity schedule.

The results were obtained using a participant-observer action research approach within the context of a Spanish language immersion camp wherein the researcher and students interacted in a manner that informed students' perceptions and responses. The use of the language instructors during the academic portion of the camp allowed for a three-to-one ratio of students to instructor. This allowed for the use of formative assessment and the ability for instructors to constantly adapt teaching for each individual student or small group during each lesson. This ability to adapt was aided by meetings held at the end of each weekend where instructors discussed and rearranged plans so that each small group met their personalized goals.

The ideal student to instructor ratio for a Weekend Spanish Immersion Camp would be one-to-one student to teacher. However, the ratio of three or four students to one councilor we had still allowed for significant support for students and flexibility.

The students in this study were nine, eighth-grade middle school students. Six of the students were females and three were male students enrolled in the same Spanish Two 
(second year) class together in the middle school. I served as both their Spanish One and Spanish Two teacher; I also served as both the researcher and lead educator for this study.

\section{Data Analysis and Interpretation}

\section{Pre-camp Questionnaire}

The first data collected involved a pre-camp questionnaire that was part of the application form completed and submitted to the researcher prior to the Weekend Spanish Immersion Camp. The purpose of the two-item, open-ended questionnaire was to elicit students' initial interests in participating in the Weekend Spanish Immersion Camp (see Appendix G).

The first item was: Why do you wish to participate in this learning camp? My intention was to find out what motivated students to request to be part of the camp. I wanted to know if students' interest were based on intrinsic or extrinsic motivation. Here are the nine students' responses to the first question:

Carlos: I feel it will increase my level of Spanish compared to people that aren't participating in the camp. I also think it will help me a lot in class.

Leah: I wish to participate in this camp so that I can get better at Spanish. I want to be able to speak fluently and I believe this camp will help me achieve this goal.

Lorita: I want to participate in this learning camp because I want have experience in a Spanish environment. I also want to learn more about the culture. Lastly, I want to expand my knowledge on speaking Spanish.

Brianna: I wish to participate to increase my knowledge in Spanish. I want to learn more about to understand it more.

John: I wish to participate to make my level of Spanish higher than what it was and I wish to learn about the Mexican culture. 
Isabella: I want to participate in the learning camp to extend my Spanish knowledge. Spanish is a language that could be very useful to know for the future and present.

Giovanni: I wish to participate because I want to learn this language and be able to understand and speak it. And maybe learn a bit of salsa [and] also learn about the food too.

Juana: I want to participate in this learning camp because I want to know more about the country and their language. I would also like to improve my speaking skills and add to what I already know.

Maria: So, I can learn more Spanish.

Based on the analysis of the students' responses, I found two main motivations for signing up for the camp; to expand knowledge of the Spanish language and to learn about the Hispanic culture. The first motivation observed across all responses was the desire to improve their communicative skills in Spanish. This intrinsic motivation was a good starting point for the Weekend Spanish Immersion Camp. Knowing our students' interests helped us to set up prompts to explicitly support their communicative skills.

For example, as a group, the three language instructors and I were mindful that each of the four weekend camp sessions needed to involve specific, clearly stated learning objectives to help students increase their oral fluency skills. Students' responses to the questionnaire reinforced our commitment to the ideal that language instructors must communicate using Spanish at all times and our ideal to reinforce students' commitment to also communicate in Spanish throughout each activity to increase their fluency.

One student stated "I want to participate in the learning camp to extend my Spanish knowledge". We believed that using certain words over and over again in 
context could help students to increasingly use the language naturally and instinctively upon prompting.

Setting up different scenarios involving cooperation was an important strategy used to motivate conversation among students and instructors. The quality of the relationships within the participant groups increased as student got used to working together as learners. Students were more supportive of each other when utilizing Spanish because they did not feel pressured or embarrassed when using the language incorrectly.

For example, by using different objects and pictures to motivate use of the Spanish language, students were prompted into conversation using the vocabulary learned during camp that was tailored to fit the themes of each day. Another student said "It was helpful because I was able to use the vocabulary over and over".

The second motivation for joining the camp found in the students' pre-camp questionnaire responses was learning about Hispanic culture. For example, Juana, a camp student, indicated that she wanted to participate in the learning camp because she wanted to know more about the country and their language. Juana's quote indicated a possible misconception that only one country speaks Spanish as their dominate language while also acknowledging her limited understanding of Hispanic cultures. Thus, students were introduced a range of cultures from countries that speaks Spanish.

Again, the Weekend Spanish Immersion Camp was divided into several sessions involving activities such as crafting, games, dancing, singing, cooking, academic skill building exercises, and watching movies. Each session was conducted by an expert on the topic they taught and targeted particular language usage. For instance, the cuisine 
session was led by a chef and the dancing sessions were led by an experienced salsa teacher, each of whom was a native Spanish speaker who thoughtfully selected words to emphasize throughout instruction.

The language instructors, researcher, and experts met in advance to plan out how to structure the sessions to better aid students' development. Prior to the development of each session, the staff provided each other with a brief overview of the cultural traditions and phrases typically used relevant to each activity to be developed. For example, the dance instructor shared information about the cultural nuances of salsa dance and identified for the group specific sets of Spanish commands to use while instructing students how to salsa dance. Songs were deliberately selected to expose students to various aspects of the cultures of different countries where salsa is danced.

Similarly, during the actual camp sessions, the students were prepared to sing by reading the lyrics of the songs together and interpreting the meaning to ensure common understanding. Then, throughout each music session, the music instructor selected new vocabulary words and taught the meaning of these new words to students and subsequently used these terms during conversations.

By role playing camp sessions in advance in a highly collaborative manner and carefully selecting the terms and phrases that would be used repeatedly throughout each session, we theorized that the entire instructional staff would be well prepared to anticipate possible student outcomes and more sensitive to interpreting student progress towards proficiency. This preparation empowered the entire group to anticipate approaches to adaptively support students' communicative skills by altering the activities 
to meet each group of three students' developmental needs.

For example, a camp participant I'll call John indicated that some of the words were difficult to remember but with the coach support it was easier for him to say and remember those terms. Language coaches helped students to memorize the words by acting out the actions related to words students expressed as being difficult to remember. In other cases, language coaches used the words in context to prompt students when these words should or must be used. However, the most important aid language coaches provided was being on hand to provide students immediate assistance when needed.

The second item on the pre-camp questionnaire was: What are you expecting to see and get during this four weekend Spanish Camp? Here are the students' responses to the second question:

Carlos: Get better at actually speaking Spanish and being involved in the Spanish culture.

Leah: I am expecting to see improvement in my ability to speak Spanish. I am expecting to get better communicating skills since you can only speak Spanish in the camp.

Lorita: During this four weekend Spanish Camp I am expecting to learn more about the Spanish culture and learn more on how to speak the language.

Brianna: I am expecting to do fun activities and lots of people helping me and speaking Spanish.

John: I'm expecting to see improvement in my speaking and understanding.

Isabella: I am expecting to see a little bit of growth in my Spanish knowledge.

Giovanni: I expect to see me grow in the language and be a lot better than I was before. Also, I expect to learn a lot and still have fun doing it. Also, just to have fun and do cool things. 
Juana: During this camp I am expecting to see myself do my best and be able to improve my Spanish. I am also expecting to learn how to cook, learn more verbs, participate in the sports, and do the activities.

Maria: I expect to get better at forming sentences in Spanish.

Two main ideas emerged from these student responses: just to have fun and do cool things and to acquire better communication skills. The first expectation students had was to have fun and do cool things. To meet this expectation, we designed the Weekend Spanish Immersion Camp to involve fun activities such as playing time, cooking, karaoke, crafting, and dancing. During these sessions, the main idea was to provide space and time for students to practice their oral skills by using Spanish-only to communicate among themselves.

Language instructors were instructed to motivate students to use Spanish as a vehicle of communication by initiating conversation. One student expressed that this a fun and effective means of support when she shared "I think it was fun because I was challenged".

Starting communicating in Spanish-only was not an easy task, at least at the beginning of the camp. Language instructors started acting out and using lot of body language to prompt students. We also had a handful of useful words for them to use repeatedly. These interactions transformed the difficulty to become what students perceived as being fun and new experience rather than a high-stakes situation where they would be afraid and embarrassed to make mistakes.

An explicitly stated goal was that throughout each activity, students were expected to use the vocabulary and phrases they learned in both the Spanish Two class 
and during the camp. At the beginning, this goal did not prove to be easy. But as we moved on throughout the session repeatedly using particular words and phrases, students were able to communicate more easily and with greater detail.

When students did not know how to say something, they had to ask instructors for oral support using body language or key words to help them get the information needed. Since many of the commands or directions were used over and over throughout each camp session, students were able to communicate with a greater degree of understanding and fluency in each successive session. As a result of cooperative structures designed prior to the beginning of the camp, I observed students helping each other by providing words and full sentences to those who did not know how to say something.

\section{Camp Observations}

The second data set I collected was camp observations over the course of four consecutive weekends. The purpose of these observations was to characterize the level of language used by camp students, levels of student engagement, group participation and relationships, task behavior, and to note instructor and students' support of one another.

Students progressed in the percentage of time that they were able to communicate in Spanish throughout the camp. At the beginning of the camp, most students were not responding as expected due to lack of language fluidity. But as students moved through the activities, students became more eager to express themselves using Spanish.

Instructors played a key role in this developmental process. The motivation and support they provided were critical to students' success. Students required a great deal of 
reinforcement and support while initiating the activities and then transitioning from thinking and communicating in English into using Spanish.

Students' level of engagement was markedly uneven across the four weekends. In other words, there were some moments during the activities that students were more engaged than others. As may be expected, the level of engagement amongst the nine students was inconsistent within each activity.

While some students were highly engaged, others demonstrated medium to low levels of engagement during in the same activity. It was apparently that that some of the activities were simply more appealing for certain students than others and that this impacted students' level of engagement. I observed that some students were primarily physically active, others were primarily mentally active, while others were a combination of both. I also noticed that even when it came to playtime, everyone engaged at different levels.

That said, students and language instructors took their responsibility seriously during the activities. Every student took part in each activity and overall, the students and language coaches exhibited a high level of engagement in the language use and development aspect involved in each of the activities.

Interestingly, an exception to this pattern was the singing activity where students showed little interest in participating. This could be because the songs they wished to learn were not age appropriate and the substitute songs chosen in place were unappealing to the students. 
Some activities required differing degrees of involvement from students. An example of this was dancing where students were required to listen and follow direction from instructor.

Overall, students did their best to fully participate in the camp activities and be on their best behavior. We never had a complaint or disagreement about the activities (which is not to say that we did not receive alternative suggestions for songs to sing).

The last piece observed in Weekend Spanish Immersion Camp is the increase of positive relationships among students. Students had already established relationships prior to the camp. I observed that the nature of the interactions that took place throughout the camp involved increasing levels of mutual support, trust, and friendship as learners cooperated to negotiate both the tasks at hand and the use of Spanish.

The Weekend Spanish Immersion Camp was designed as a learning resource to support positive behavior and increase participation in communicating in the target language as a learning community. The activities set up for the camp had the intention of providing students opportunities to support each other while interacting and completing the intended objectives. Indeed, it seemed that spending more fun time together encouraged students to rely upon and accept each other's input. It appeared that students gave each other support and were more open to receiving the support of their peers in comparison to how they behaved in the formal education Spanish Two course prior to the camp experience. 


\section{Interviews}

The third data set I collected was individual interviews with students conducted during the last day of the camp. Each interview was recorded and videotaped to observe students' facial and body expression for deeper analysis. We set up a special interview area where we had some artifacts and samples related to specific tasks and activities completed during the sessions. The purpose of these materials was to prompt students to remember those days the activities were conducted that involved these artifacts. The interview did not involve set time limits. I expected each student to take their time to think before they responded each question. The interview did start with direct questions but I then worked to establish a comfortable rapport by encouraging students to take the conversations in their own directions. See appendix $\mathrm{H}$ for the interview prompts.

\section{Presentation of Results}

The findings presented herein are organized around three open-ended research questions. The first question was: To what extent do Middle School Spanish students perceive that Spanish immersion camp instruction serves to assist retention of world language communicative skills? The second question was: What kind of activities from the Spanish immersion camp effectively support Middle School students learning a world language? The third and last question was: How does the Spanish immersion camp environment influence Middle School students' perspective of their world language learning process? What follows is a description of the data that informs each of the questions. 


\section{Interview Data that Informs the First Research Question}

What follows is a report of the data that informs the first research question.

Again, this question was: To what extent do Middle School Spanish students perceive that Spanish immersion camp instruction serves to assist retention of world language communicative skills?

The first question asked of students was: On a scale one-to-ten (with one meaning a little and ten meaning a lot), rate how much did you like the Weekend Spanish Immersion Camp? One student gave the camp a rating of seven, four students rated the camp as an eight, three rated the camp as a nine, and one student gave the camp a ten. The focus code(s) assigned to responses to this interview item are: academically beneficial, fun and wish to comeback.

Every student indicated a different degree of approval; however, a major finding of this study was that all students gave a positive approval rating to the program. All nine students reported that they liked the Weekend Spanish Immersion Camp to some degree because they found it fun and academically beneficial. One student enthusiastically stated "I love it so much; I will say ten. I enjoy the camp and I want to keep coming".

Students were asked: Among all the activities we implemented during the Spanish immersion camp, which was your favorite activity? One student indicated craft as his favorite activity. Three students stated that their favorite activities were the games we involved in the camp and other three students pointed to cooking as their favorite activity in the camp.

In my professional experience most of my middle school students tend to prefer 
physical activities, even in languages classes. That said, to my surprise, two of the students indicated the academic sessions were their favorite parts of the camp experience. Maria said "I liked learning new vocabulary because I feel like I can say more", while Lorita claimed; "my favorite activity was academic because I like when we had to write paragraphs or stories using the vocabulary". The focus code derived from responses to this item is prefer hands-on learning.

The next finding relates to examining the effectiveness of the academic sessions. The aim of each academic session was to provide new word knowledge to increase students' communication skills. These sessions provided twice daily throughout the camp schedule during involved dividing the group of nine students into small groups of three with one camp language instructor assigned to each group. Students worked for one hour learning new vocabulary that was expected to be used during the weekend. The vocabulary introduced each weekend was then used in each successive weekend session so that the students iteratively built up new vocabulary skills.

During each academic session, students were given a set of thoughtfully selected vocabulary words to learn and then use to create a short paragraph or story. Students were also encouraged to select new words to add to the list. Students were then asked to find a way to integrate the new words into their existing body of vocabulary throughout the day.

All staff were trained to find ways to use the vocabulary learned in all activities. This was done both prior to the beginning of the camp when the initial set of key words and phrases were identified and at the conclusion of each weekend as student selected 
new terms to add to the growing vocabulary list.

I asked students; On a scale from one-to-ten (with one meaning I recall nothing and ten meaning I can recall everything), rate the amount of content you believe you remember from the academic portion of the Spanish Immersion Camp? One student rated six which was the lowest and other students rated nine which was the highest rating number. These ratings indicate that at the end of the four weekends, students claimed they still remember most of what they learned during this experience.

I asked two questions with the goal of understanding how students perceived how the Spanish immersion camp helped them retain world language communicative skills. The first question was: Based on your personal experience, how helpful did you find the academic activity? Here are their responses:

Maria: It was helpful because I was able to use the vocabulary over and over.

John: It was fun. Some of the words were like difficult to remember but with the coach support was easy to say it.

Juana: I don't know, I think it was good.

Leah: It was really fun to learn new vocabulary and use them during cooking.

Lorita: I think it was fun because it was challenged.

Giovanni: It was fun for me. I think I can say more now.

Isabella: Uh, I think it was ok. The teacher was very patient and helpful.

Brianna: It was fun and made me think a lot. I liked it.

Carlos: It was a new way to learn more words. It wasn't fun as other activities but interesting.

Their responses demonstrated that while none of the students disliked the 
academic portions of the activities, they each liked this aspect of the Weekend Spanish immersion camp to a different degree. That said, their responses indicated that every student recognized the practical importance of this aspect of the camp. The focus codes for this item are practical and fun way to learn.

To obtain a deeper understanding of their perception of the need to implement Spanish immersion camps as supplemental academic support, I asked a second question: What do you remember or what was your experience on the academic activity?

John: It was good. It was like...uh, I don't know how to explain it ... but I liked to work in groups and get support from each other.

Leah: Ok the academic portion, we just learned verbs... umm... like I remember gritar (it means shout). Well I mean, it wasn't like fun. It wasn't supposed to be fun....ahh, but I got time to work on what I needed to work on. I can pronounce better now. I have a friend who doesn't feel confident in saying it because she can't pronounce it easily so this activity helped her and helped me because now, I can say it better instead of saying it wrong.

Lorita: Umm, I feel like umm like it really helped me get the concept of what and how to put things together. Yeah, it was fun.

Giovanni: I liked it a lot because it was more hands on. It was more like one-onone than in class. In class, it's hard to get one -on-one with the teacher but I do like the immersion camp cause it's more one-on-one. You helped me get what I'm saying wrong and make sure I'm saying it right.

Isabella: We would practice learning new verbs and then we would have to write sentences with them.

Brianna: The academic part was good, like it helps probably a lot with just how to conjugate words or verbs.

Carlos: Focused and concentrated and it felt good to be like one-on-one.

All nine students' statements indicated that Weekend Spanish Immersion Camp was a helpful academic support because it provided them opportunities to have their 
hands on and work in small and large groups. The academic activity also helped students to improve language pronunciation while interacting in small groups with language instructors. The focus code for this item is increased confidence.

During the Weekend Spanish immersion camp, I asked the students to please give an example and explain the type of material they have learned during the academic portion of the Spanish Immersion Camp. Their responses are summarized into two focus codes: learning new verbs and building sentences.

Students were asked to select a set of verbs they wanted to learn in each academic session; each group limited the number of words they wanted to learn. Some groups picked more words than others. This freedom to select their own target words led to a sense of competition with some students highly motivated by the sense that they did not want to be left behind by the other groups. Once the table groups selected their lists of words, the language instructors helped them learn the new words by pronouncing each term, interpreting the meanings, and supporting their understanding of when to use each term by using the word in a sentence. Most of the time the sentences used were related to ideas that middle school students experience within their social life.

After mastering the basic understanding of the meaning of each word, members of each group were asked to use them in sentences in different tenses. Later, these sentences were placed together until the group was able to construct stories that integrated these new words with their existing body of knowledge. The idea behind this activity was to show students that whatever they learn can be transferred to real life situations. For example, one participant said "At first it helped me to learn some new 
verbs and it helped me change the verbs so that I could use them in different kinds of sentences."

Students indicated that they found this approach to be supportive for preparing them to communicate during the activities that followed using the new words. One student commented "I remember learning a lot how to build sentences and how to analyze them and put them together in a good way where people could understand what I'm trying to say. And I could remember how to make sure the sentences make sense in way like umm, like how to conjugate them and put them in ways that say in the past or in the future or right now".

Next is a presentation of data elicited in response to the question: Which activities do you believe helped you learn, practice, and understand Spanish the most and which helped you the least? Explain. Again, the Weekend Spanish Immersion Camp involved a broad range of entertaining activities designed to support students to increase their communicative skills and confidence as students of the Spanish language. For example, the camp involved fun activities such as crafting, games, dancing, singing, cooking, and watching movies.

Even though they were fun, students agreed that fun activities alone were not going to help them achieve the desired increases of their communicative skills. The focus code derived from their responses was forced quick thinking.

Leah expressed that even during fun activities, it was important that students were asked to use the Spanish language to communicate. In fact, in one way or another, all of the camp activities involved language usage. As one student reported "Umm well, things 
like crafting and dancing [alone] is obviously not going to help me learn Spanish but more like, I mean the culture of it, so that really helped me. I know that umm for instance when we ran like from there-to-there that helped me because that forced me to think quickly about what I had to say, so that way I had to like, you know, tap into that so I thought that was pretty cool”.

All of the camp students agreed that although the sessions involving reading, writing, and using new vocabulary words in sentences were not their favorite activities in the camp, these were the most helpful activities to increase their Spanish language fluency. Maria said that reading and academic support activities were the most helpful for her because students had the opportunity to work one-on-one. For her, the least liked activity was a particular game we played because she said they were just having fun and yelling out the numbers. She also added, "but it was still really fun to play the game".

The students acknowledged that they had fun playing games, but as Leah stated, students agreed that the small groups and one-on-one interactions were more effective in helping students to build their foundational knowledge of the Spanish language that they could then use during the activities. For example, another camp participant said "I would say the one-on-one [was the most useful] but all of it really helped because it was Spanish and you were all immersed in it. The least [was] probably just dancing or singing. It was still fun because you get to learn, like, the culture but..." The focus codes I derived were dancing and singing were less successful and importance of one-on-one. 


\section{Data that Informs the Second Research Question}

What follows is a presentation of the data that informed the second research question. Recall that this question was, What kind of activities from the Spanish immersion camp effectively support Middle School students learning a world language?

\section{Activities Students Preferred.}

Students were asked a series of questions and their answers were audio recorded to ensure transcript accuracy. The first question was: What was your favorite activity?

The Weekend Spanish Immersion Camp involved six different activities designed to scaffold world language learning. Out of the six activities (academics, crafts, cooking, games, dancing and singing), students claimed they preferred the academic activities, crafts, cooking, and games sessions. The two activities that students said they did not enjoy were dancing and singing. In the next paragraphs, I will explain how these sessions were arranged.

The academic sessions involved reading short stories, writing sentences and paragraphs and learning new vocabulary words and verb tenses. Students were divided into three small groups comprised of three students and one language instructor. The ratio of three-to-one was chosen based on the rationale that this arrangement would provide more opportunities for students to interact with their language instructor and each other in comparison to working as a larger group of nine students with three instructors.

Each weekend, the session started with students selecting a set of words to be learned and used during the camp. The intention was that the students would build on 
this set of vocabulary words each of the four weekends.

Again, the language instructor was charged with finding opportunities to fluidly integrate the growing set of terms as naturally as possible into daily conversation, thus modeling and encouraging students to use the words in sentences in both casual conversation and in structured activities. All these activities were carried out exclusively in Spanish.

For example, after exploring how the words were put together to build sentences, students were asked to put the sentences together to write short, personal fictional or nonfiction stories. Students were asked to verbally present their personal stories to the group. This type of interaction motivated students to be increasingly more creative every time it was their turn to demonstrate use of the new terms embedded within their pre-existing knowledge.

In the game session, we focused our attention on two types of activities; physical and mental. The game sessions were set up in the school gym. Since we had nine students, we usually had two or three teams competing against each other. What follows is a description of one of the games we played during the game section of the camp and the focus codes that emerged from students' responses to the game sessions.

We had six tables set up in the gym. Three tables with envelops on them were facing the other three tables separated by a distance of fifty yards. In the envelopes, students found sentence strips that were cut up into single words and phrases. The sentences made up a story. The students' job in this game consisted of carrying one piece of the short story at the time to the table in front and arrange the words and phrases until 
they put together the whole story. A team of three students were assigned per two tables.

All students reported that they loved the competition and that they learned to trust and support each other to build the short story. The focus codes derived from student responses to the games are competition and trust building.

Nine students indicated that crafting was one of their preferred activities. During craft sessions we worked on building piñatas for the party we had on the last day to celebrate the ending of our camp. We presented three types of piñatas and provided all the materials and tools needed to accomplish the task of constructing and decorating piñatas. We divided the group into two teams. One group of students decided to build a guitar piñata while the other group of students decide to build the donkey piñata. The camp instructor provided the directions for making the piñatas in Spanish and read the directions out loud. Students listened to the directions and follow them to cooperatively build each piñata. The instructor's job was to support them verbally using Spanish only. Students used their Spanish language knowledge and their reasoning to plan the construction and decorate each piñata.

In this craft activity, students were given the opportunity to build a piñata on their own. Students were very creative and did their best. Once the piñata was completed, students filled them with sweet goodies and small toys. While working on their piñata, students used Spanish to negotiate design and material to use. All nine students enthusiastically reported that this creative approach involving the use of language to cooperatively create art was very effective. The focus code for the crafting activities is creativity. 
We hired a Mexican chef for the cooking session who included cuisine from Peru and Panama in the dishes she helped students to prepare. Students took notes on the recipes and asked the chef questions for clarification throughout this hands-on activity that involved setting up the ingredients and preparation of the different dishes. Two students reported that they tried the recipe at home too.

After the food preparation, students took turns setting the table before each meal. The chef was part of the communicative process by providing opportunities for students to be involved in Spanish conversations as students carried out the directions that were provided in Spanish to properly set the table. Leah commented that cooking was really fun because students learned new recipes and had the opportunity to try new food.

From our observation during this activity, we observed students very interested in the preparation and ready to help on anything they were asked to do. Per our conversation during the activity with all students, we heard them saying that this is a new experience for them. Some of them indicated that they never had a chance to cook but they expressed they had fun doing it. The focus codes are new experiences and fun.

\section{Activities Students Did Not Prefer.}

Even with the students' apparent willingness to learn and the staffs' pre-

disposition to do their best to ensure that each activity was conducted in a fun way, not all activities were equally liked. As mentioned above, the least liked activities were dancing and singing.

The researcher hired a salsa instructor for the dancing sessions that involved instruction conducted in the target language for two-hour weekly lessons offered on each 
of the four weekends of the camp. We set the gym up with powerful speakers and festive Hispanic decorations and explicitly invited students to have fun while learning culture, language, and dance. The pieces of music selected were from different Hispanic countries including salsa from the Caribbean. The salsa instructor did a great job directing the dance moves in Spanish. Indeed, the students appeared to enjoy the lessons and have fun while dancing. Here are some comments made by the students:

John: I danced three new types of dances. I like [that] if I see someone dancing that dance, I'll know exactly what it is and like follow those moves.

Lorita: The portion was also fun. I wasn't here for some of the them because of softball but the times I was here I learned more about the dancing and the culture.

As the salsa instructor taught the moves, she explained the roots of salsa and the influence of African instruments and movements to this type of rhythm. Some students related that they liked the idea of learning how to dance salsa and learning about the various cultures that salsa emerges from, however, because of a lack of prior exposure to this type of music, the students shared that they experienced a low level of emotional response to the music. The focus codes are like to dance and never danced salsa.

Another session all students did not like was singing. The singing lessons were conducted by an instructor who has a strong voice, rhythm, and feeling when singing, especially Hispanic songs. This music instructor selected a series of four songs to be sung by students during the weekend Spanish immersion camp. Most students verbally expressed that they did not like the type of music selected for this camp. From their comments, it was apparent that this group of students were more attracted to modern songs sung in English than to Hispanic songs sung in Spanish. 
Our idea was to create a karaoke environment, but maybe because of the relatively young age of the students, they were not familiar with karaoke or willing to take the risk to sing in front of their peers. For example, Carlos indicated that his least favorite activity was the singing because it was awkward to sing in a group. We did our best to encourage the campers to sing, however, we did not succeed. The focus codes that summarizes their responses are "never sang in Spanish" and "feeling awkward".

\section{What Students Liked About the Preferred Activities.}

As mentioned above, the activities students preferred the most were the games, cooking, academic activities, and crafts. When students were asked to indicate in one word or phrase how much they liked these activities three students rated the activities as "fun" (Lorita, Carlos and Leah). Two students said they ranked the four activities as their favorites because they were "new experiences" (Carlos and Giovanni) that they thought were effective approaches to language instruction.

Students found the camp a little different from classroom instruction. All students indicated that they liked the experiences of creating sentences and then paragraphs when putting the words and verbs together and ending with a small personal or familiar story. They shared that they liked when the learning was personalized. The focus code is personalized learning.

Again, the camp instructors introduced students to games from other countries. Campers commented that they enjoyed integrating the use of Spanish as they learned the rules and how to play each new game. As their self-confidence grew and the campers felt comfortable, they reported that the learning extended beyond the confines of the camp. 
Four students indicated that they taught the new games to their friends in their neighborhoods and that they "had a blast" sharing the games they learned at camp with their friends and families. The focus code is confidence.

\section{Data that Informs the Third Research Question}

The third research question was: How does the Spanish immersion camp environment influence Middle School students' perspective of their world language learning process? Based on our experiences as educators, we knew that environment plays an important role in the learning process. As a group, we believed that the Weekend Spanish Immersion Camp would not work in the ways we intended it to influence learning if we did not thoughtfully arrange each learning environment that the camp would operate within. Therefore, we purposely arranged each specific setting in particular ways. For example, we played welcoming music at the beginning of each camp day to set the tone for this informal learning experience.

As mentioned in chapter three, the camp involved four major areas within the school building where learning occurred. We set up these places with thoughtfully selected materials and supplies useful to support world language learning such as festive Hispanic decorations in the hall corridor where the salsa lessons occurred and carefully selected pictures along with words written in Spanish to orient student understanding and communication to the activities such as writing personal stories and crafting.

Furthermore, we consider the role of body language as a supportive teaching and learning tool. For example, we explicitly modeled maintaining positive expressions to create an environment wherein it was safe to take risks and make mistakes. 
We knew that requesting students to give up four weekends was not going to be an easy task. To do this, we needed to create an atmosphere that felt different from the formal school day. Environment involves not only features found in a physical location but also the nature social interactions. The staff camp was committed to create a welcoming environment and actively encouraging enthusiastic participation.

To find out whether or not students thought that the environments we created made a difference to their learning, we asked the following: Considering your interactions with the teacher, assistants, and your fellow classmates during the course of the Spanish Immersion Camp, how did the environment of the Spanish Immersion Camp help you learn Spanish? Here is a selection of responses students provided:

Maria: Umm I really liked the environment that was around me because I hadn't met your daughters before and once I did, they were like really welcoming and really nice to me.

John: It was more like the people who were here wanted to learn Spanish so I didn't have to feel embarrassed if I messed up.

Juana: It helped me learn Spanish cause we didn't do things a lot that we do here but we did a lot of stuff, writing down stuff. Speaking and writing helped me.

Leah: Well like if I said something, they just helped me instead of being like you got it wrong.

Lorita: Well we all like it, like forced me to work more on my Spanish and like really listen, like take in what you're saying, like what people are saying and stuff, like when I like one-on-one interaction, like I have more time, like help me.

Giovanni: Feels like I can understand a lot more and when the other kids are struggling I can help them out a little bit more because I know a little bit more after the immersion camp because it helped me grow more and understand what I need to do and make sure how I can speak the language correctly.

Isabella: It helped me learn Spanish cause you could only speak Spanish while we were in the camp. 
Brianna: Well, like it forced you...kind of to speak more Spanish, and it challenged you to do that so that's probably how it helps like you weren't just like all of a sudden speaking English cause you couldn't.

Carlos: I guess we were all doing the same language and everything and we are all in the same boat or activity and stuff like that yeah it helped me.

According to students' statements, they perceived the social environment as being highly supportive of taking risks and demonstrating learning on an iterative basis. That is not to say that students found the activities involved in the camp to be easy. Indeed, students explicitly stated that the camp was a challenging experience because for the first time as students of a foreign language, they were invited to use Spanish as their only communication vehicle. That said, the students agreed that they did not feel embarrassed to try, to make mistakes, and be corrected because everyone was doing the same thing as they were using the language to communicate with the support of the language instructors and peers. The focus codes are welcoming, one-on-one, "speaking and writing, and social support.

One student indicated that they “didn't do things a lot in classes that we do here in the camp". Indeed, the language instructor has much more time and flexibility to incentivize, encourage, and support the individual campers on a one-to-one basis as they worked to properly put words together in manner that is difficult if not impossible for one teacher to do in a large class in a 50-minute class period. Another student stated that the camp environment "forced them to work more on their Spanish" because their instructors were providing close oversight on an on-going basis that encouraged constant participation. This struck me a key observation that points to the fact that students who 
fail to participate on an equal basis can more easily be overlooked and not challenged in a large group setting in comparison to the intensive environment a small immersion camp can provide. The focus codes are social support and Spanish only.

This observation strongly supports Snow, Padilla, and Cambell's (1998) argument that immersion programs are the most effective approach to world language instruction. Here, we observed students helping each other by providing one another clues or words as the fellow campers struggled to express themselves. Students both said and demonstrated that this type of interaction made them feel empowered. One student commented "when the other kids are struggling, I can help them out a little bit more because I know a little bit”.

In the words of one student, the Weekend Spanish Immersion Camp learning environment helped students to "grow more and understand what I need to do" as Spanish learners. Because they could only speak Spanish while we were in the camp, all of the students came to the camp every weekend willing to grow their Spanish communication skills. They demonstrated with words and actions that they understood what we trying to do with them. For example, even though some of them had weekend sports commitments, students stated that they preferred to commit to the camp because they knew they would have more Spanish communicative skills after the immersion experience.

I also wanted to know how the environment stimulated the campers to continue learning more Spanish. Therefore, I posed another question to them: How did it change the way you learn Spanish? The following is the feedback I received from students: 
Maria: It changed me or it changed the way that I look at learning Spanish because I know that we aren't just learning a language, we are learning like an entire culture and how they like, play games and cook and like, how they, like...say sentences and all that.

John: It made it easier because now I am in a better environment for learning. It's more quiet and I have more like...I have more like, time, yeah.

Juana: I was able to learn a lot more here because there weren't the big groups. It's good cause I think that, since in class we have a lot of people who are a little chatting and they hold us back. I think, I was able to learn a lot more here than I do in class.

Leah: Umm...well, I can pronounce things better umm...I know how to form sentences better. I know more verbs, yeah.

Lorita: Well like okay, so it like helps, like me, like really focus on piecing things together that help me understand like what is being said or written.

Giovanni: It made me look more at it and more in different ways and make it more exciting and more.

Isabella: It made me change because I had to figure out how to say stuff in Spanish instead of English.

Brianna: It's kind of just challenged me more to just speak it instead of just asking in English what does this means and stuff.

Carlos: Just being feeling immersed in it instead of talking to all of the class at the same time and learning that...that's how I learn better just one-on-one.

The conversational Spanish immersion environment had a great impact on the way students perceived how to learn a world language. Their experience can be summarized with these focus codes; changed me, work in small groups, more exciting and challenged.

In summary, all nine students reported that they felt that the Weekend Spanish Immersion Camp environment played an important role in their learning world language. They agreed that the safe, fun environment stimulated communication in Spanish and that 
each activity and space promoted a sense of welcoming to take risks and try new things. The setting of each room was intentionally decorated to prompt students in ways that were fun and interesting while at the same time the camp guidelines maintained a sense of rigor by asking students to use the target language only, an element that students confirmed promoted learning.

Next, I will present my interpretation of my finding related to the three interview questions asked of students after the Weekend Spanish Immersion Camp. These interpretations are presented in the order of the research questions.

\section{Summary of Findings from the Interviews}

\section{Summary of Findings Related to Question One.}

Recall that the first research question was: To what extent do Middle School Spanish students perceive that Spanish immersion camp instruction serves to assist retention of world language communicative skills? The following are key responses found in these data related to the first research question.

The first key point students raised in their responses to this question was related to the instructional strategy of having students working in groups. Students related that working in groups provided them with a high level of motivation.

A second key point made by students was improving confidence when using this world language. Students claimed that the environment, the support of the instructors, the high level of personal motivation, and the benefits of working in small groups helped them feel confident and able to express their thoughts and ideas without fear of derision from their peers. 
This last point is important given that, in my experience as an educator, when students feel confident and willing to speak without the fear of making mistakes, they will explore ways of placing words and verbs. Again, learning to conjugate Spanish verbs is not as easy as conjugating verbs in English. In English verbs have two conjugated forms (e.g., work-works) in the present tense. In Spanish, there are six different ways to conjugate this word (trabajo-trabajas-trabaja-trabajamos-trabajáistrabajan). Understanding the multiple ways to properly conjugate a word demands more effort, support, and practice. With the instructors' support and that of their peers, students were encouraged to explore word order and verb conjugation. All students reported that this activity increased their communication skills and confidence in their ability to properly understanding the rules of the language.

Another key point found in the data related to a sense of learning a world language hands on. A central goal of the camp was to provide opportunities for students to focus on this negotiation of meaning to obtain better and faster results in learning correct use of the language. Learning a world language means using a variety of tools that will stimulate reflexive use of language skills. For example, the camp involved the use of objects and pictures to trigger communication in the target language. The immersion aspect of the program involved focused concentration on particular topics involving the use of specific language that encouraged students to use Spanish to communicate thoughts and ideas to their peers to negotiate meaning in a highly practical manner. 


\section{Summary of Finding that Inform the Second Research Question.}

The second question was, what kind of activities in the Spanish immersion camp effectively support Middle School students learning a world language? We planned each camp activity to involve full immersion in the Spanish language. All students agreed that the camp was a helpful addition to the school world language learning program. One student commented that the camp was "helpful" and another student mentioned "I would say that the camp goes more in depth into things since we have more time to actually learn Spanish. In class, we just go over the basic stuff and sometimes we go in depth but normally it's not as much since we don't have much time".

The purpose of the camp was not only to impart knowledge over the course of the four weekends, but to also establish a better relationship among students and instructors involved in the Spanish Two program at school. As one student shared, "We grew together and learned to persevere and be supportive of each other." Another student commented that the camp "really impacted me... it was fun and made all of the students to bond and get to know everyone else".

The emotional bonding that occurred during the camp positively impacted classroom interactions in the Spanish Two class as well. Two examples of this impact were students actively using the Spanish language communication skills learned in camp and the camp students asking each other for help first rather than coming directly to me, the teacher, every time they needed assistance with sentence construction, pronunciation, or word choices.

I asked all nine students to compare the content they learned during the Weekend 
Spanish Immersion Camp to what has been covered in class. Every student indicated that the depth of content they learned during the camp was much greater than the depth of content they were able to approach during regular classes. Here are their statements;

Maria: Umm, I would say that it was a lot more advanced but umm I think that's good because it made me learn a lot more.

John: I've learned more in the Weekend Spanish Immersion Camp cause it's more like what I need to learn and it's not like where everybody and trying to get everybody at the same time.

Juana: I would say this camp was higher than what we learn in class. I definitely understand more; now I know more verbs and how to put them into longer sentences.

Leah: I know how to pronounce [words] better instead of like looking at it and saying it but not necessarily having someone to correct me...It's kind of like, you go at your own pace and you learn a bunch of verbs and put them in sentences. This class you kind of do like the material, so...

Lorita: I've experimented [with] a lot of knowledge more because I am forcing myself to practice Spanish [by] saying it.

Giovanni: I feel like I can understand a lot more from what if my friends are trying to talk in Spanish. I can understand a lot more and if I need to talk to somebody in Spanish, I can say a lot more things... after the immersion camp.

Isabella: We use a lot of the same verbs and I learned some new ones. We, in both scenarios, we try to put tenses together.

Brianna: The academic part was good. Like it helps probably a lot with just how to conjugate words or verbs.

Carlos: Yeah, a little bit but it was more one-on-one you could actually focus during the camp and it was all in Spanish so yeah.

While observing students' interaction with the language instructors I noticed that students' sentences related most often to things or actions they usually do in their daily life. Thus, this highlighted the importance of making the learning contextually relevant 
to students.

Students were given the freedom of using the verbs in the tense they choose to express their thoughts. instructors and students indicated that giving students freedom of choice was a key element to motivate students to expand their own learning.

The following summarizes the perceptions of this group of nine students of what Weekend Spanish Immersion Camp experiences can do for students.

- Weekend Spanish Immersion Camp is a more advanced instructional resource that world language teachers can use to motivate students, enhance learning, and reach higher communicative language level.

- Weekend Spanish Immersion Camp can provide one-on-one language support; students will have someone to correct them in situ.

- Weekend Spanish Immersion Camp is a powerful approach to interactively building up knowledge and usage of new words in context; we use a lot of the same verbs, over and over, until it becomes part of their natural statements in daily conversations.

- Language instructors do not force students to use specific words. Students push themselves to speak in Spanish. Lorita indicated "I like forcing myself" to speak the language.

- The interaction with each other helps students to use more words and verbs to put them into longer sentences. Therefore, increase their language level, speed and natural language flow.

- Students indicated that Weekend Spanish Immersion Camp is an instructional 
resource that can provide what students need to learn in a safe and motivating environment.

\section{Summary of findings that Inform the Third Research Question.}

The third research question was: How does the Spanish immersion camp environment influence Middle School students ' perspective of their world language learning process? When we set up and organized the road map of Weekend Spanish Immersion Camp, we thought about bringing to the students a new way to examine ideas related to Hispanic cultures, worldviews, and how they interact with each other. Language is not simply a matter of putting words together. Rather, learning a new language within the context of an immersive experience involving interactions with native speakers of the target language provides opportunities for learners to gain an understanding of why Hispanic people state ideas in a certain way that could be totally different from the way ideas are expressed within English-speaking cultures.

Literal translation of words is not the only way native speakers communicate. Sometimes, the position of words, intonation of a word, and body language may change the meaning of a phrase, sentence, or word. All nine students agreed that learning Spanish in an immersion camp is "more exciting" and highly nuanced than learning that typically takes place in a regular classroom. They indicated a growing awareness that learning a new language is not simply about learning new words, phrases, and verb tenses. They became increasingly aware that learning a world language provided them with access to explore "entire cultures" and to distinguish nuances between sub-cultures. 
Weekend Spanish Immersion Camp provide students with plenty of time to interact with each other. Students were allotted sufficient time in small groups to understand and gain communicative skills through their interactions with their peers and language instructors. As one student commented, the Spanish immersion camp "just challenged me more".

At the end of each weekend, the camp instructors and researcher met to analyze the learners' progress and to prepare the lessons for the coming weekend. Based on formative assessments of the students as individuals and in their respective groups, we were empowered to provide students with more challenging things to do the following weekend based on the students' actual levels of achievement. We knew that each small group was moving at their own pace, therefore the material to be used with each group the following weekend needed to be slightly different.

We observed throughout the four-week immersion experience that the small ratio of three students per every one instructor assisted each team to be more productive learners. We arranged enough material for each group to be constantly challenged to increase their level of understanding and speaking Spanish in unexpected situations that demanded the natural development of skills. One student expressed that one-on-one support in actual conversations rather than pre-arranged exchanges improved their fluency. As one instructor observed, students "focused their attention on piecing things together to construct new ideas and new learning experiences" in highly reflective, collaborative ways. 
All students observed that the Weekend Spanish Immersion Camp provided an environment that cannot be achieved in a typical classroom setting. Indeed, this is true due to the many obstacles that unfortunately middle school students are all too aware of such as short class periods, large class-sizes, only one native speaking teacher (in the best scenario case), a lack of funding to purchase sufficient materials, and a lack of willingness and commitment from students to engage with enthusiasm based on intrinsic motivation for learning together.

\section{Limitations of the Study}

One of the biggest limitations of this study was the small number of students in the program. Only nine students, which represent one-third of students enrolled in second year Spanish class, participated in the Weekend Spanish Immersion Camp. Although the outcomes achieved within this small group were great, I would like to know what result could be achieved if $100 \%$ of the Spanish Two students take advantage of the

program. In particular, it would be interesting to examine how the positive outcomes we saw such as increased confidence, fluency, bonding, and willingness to take risks would affect the dynamics of the formal Spanish Two course.

Another limitation was the brevity of the camp. While the four weekend Spanish Immersion Camp did help students to increase their oral communication skills, given the overall brevity of the camp, this experience produced limited achievement. I wonder what would happen if this program were extended beyond the four weekends. Also, I am the students' regular Spanish classroom teacher in Middle school. We have already established certain connection due to our classroom interaction. I wonder, what would the 
research be like if someone conducting the research does not have a close connection.

\section{Summary}

Chapter four presented an students' responses to the pre-camp questionnaire, camp observations, and interviews. Students' responses to the pre-camp questionnaire demonstrated a high level of interest and motivation amongst the nine students who signed up for the camp with the express interest of improving their Spanish language skills.

In comparison to students' usual performance in Spanish Two classes, the camp observations revealed an increased level of student engagement while using Spanish as their vehicle of communication to negotiate fun tasks and improving relationships among students, camp staff who served as language instructors, and with me, their teacher.

In the next chapter, I will present an analysis of the results presented in the form of themes that inform each of the research questions. Chapter five will present a discussion that will situate the findings with the context of the literature I read regarding language immersion programs and within the larger context of world language education. Recommendations for practice will be provided followed by recommendations for further research and the conclusion of this piece of dissertation research. 


\section{CHAPTER FIVE}

\section{DISCUSSION AND CONCLUSION}

This participant-observer qualitative action research examined the perceptions of a group of nine, second year Spanish middle school students as they prepared for and attended a Weekend Spanish Immersion Camp. The purpose of this study was to conduct qualitative participatory observer action research focused on a four Weekend Spanish Immersion Camp involving a group of middle school students in their second year of world language instruction to elicit and describe how, if at all, campers perceived that the camp experiences contributed to the retention and development of their Spanish language skills.

Again, the context of this study was a Spanish immersion camp held on the same middle school campus where the nine students attend school and I work as a Spanish teacher. The camp was developed as a part of this dissertation study and involved designing and testing a variety of teaching techniques to extend classroom learning.

The camp environment involved activities intended to motivate the development of oral language skills through one-on-one and group conversations in Spanish. The immersion experience involved traditional Latin American cooking, salsa dancing, singing, theatrics, creative arts, and academic skill building where Spanish was the primary vehicle of communication. These activities were intended to create an environment for students to draw on their knowledge of vocabulary and formal sentence structures to actively integrate new words and phrases to communicate in the target language, to foster self-confidence while learning the target language, and to foster the 
development of collaborative, trusting relationships amongst students within the context of an enjoyable learning environment.

The study focused on students' perceptions and their understanding of how the language immersion camp experience contributed to their world language education. The study employed the lenses of social constructivism and situated learning theories to analyze how, if at all, the immersion camp students perceived that the camp environment and activities motivated and supported development of their Spanish language fluency. This study involved three open-ended research questions:

1. To what extent do Middle School Spanish Two students perceive that Spanish immersion camp instruction serves to assist retention of world language communicative skills?

2. What kind of activities from the Spanish immersion camp effectively support Middle School students learning a world language?

3. How does the Spanish immersion camp environment influence Middle School students' perspective of their world language learning process?

Chapter five will build upon the thick, rich description from a student-centered perspective presented in chapter four by providing a synthesis of the findings and interpretation of the results along with a discussion of how the results inform the three research questions. I will then discuss three key implications of the findings followed by suggestions for future research. Finally, this chapter and entire dissertation provides a summary of what is to be learned from this study and a conclusion. 


\section{Analysis of Findings}

\section{Themes that Informed the First Research Question}

The first theme that emerged related to the first question, To what extent do Middle School Spanish Two students perceive that Spanish immersion camp instruction serves to assist retention of world language communicative skills, is that students appreciated the practical nature of the camp that required the use and development of conversational Spanish skills as a vehicle to increase their world language communicative skills. For example, as described in chapter four, one student reported "I liked learning new vocabulary because I feel like I can say more". This was a theme echoed by the entire group throughout the experience. The focus codes I found for the first questions are academical beneficial, fun and wish to comeback.

The learning and teaching material used throughout the camp were specifically tailored to meet the developmental needs of the group and for individual students. For example, all nine students were told that the words they learned during the academic sessions would be the words students needed to use to participate in the camp.

The following comment made by one student echoed sentiments expressed by every student at various points throughout the camp, "I liked it a lot because it was more hands on." The practical nature of the structure of the camp reinforced the importance of what they were learning because they saw for themselves how their new found knowledge could be used both within and outside the classroom setting.

This finding is in accordance with Snow, Padilla, and Campbell (1988) who argued that because immersion models require students to learn in groups and forces them to use the target language to make meaning, immersion models allow many 
opportunities for English speakers to develop and maintain world language skills useful for supporting learners' independent language development skills. In fact, the level of improvement students perceived was directly related to the amount of time spent in the program practicing with their peers and mentors. As the weekends progressed and students repeatedly heard and used the same words and phrases spoken, they claimed that their confidence grew over time as they were increasingly able to use those words and phrases with fluidity.

A large motivator for the development of the Weekend Spanish Immersion Camp structure was based on my own personal struggles in learning a world language when I was younger. Although I received a lot of in classroom instructions, I did not have the ability to practice actual conversations. The closest I could come to practicing conversational English was going to the tourist laden parts of the city and trying to have a conversation with strangers. Students articulated that they appreciated that Weekend Spanish Immersion Camp created opportunities most world language learners lack, an environment where they could use the target language to engage in conversations about real situations they have to solve.

All students mentioned that they felt that the strong emphasis on focus and concentration, specifically the one-on-one attention they received from their instructors, was a major factor in their learning of communicative skill. LoCastro (1989) indicated that large classes leave less time and opportunity to provide prompt individualized feedback to students. These increases make it more difficult for teachers to monitor individual student's objective achievement and tailor instructional responses to meet each 
student's needs. At the same time, students have less opportunity to be active students in class and to demonstrate or correct their understanding of content knowledge in a timely manner.

The controlled setting allowed students to ask for help and instruction in real time so as to not pull them away from the moment because they cannot find the proper word to describe their thoughts. Similarly, Pufahl and Rhodes (2011) found that intensive, immersive instruction in the target language and a high degree of student interaction produce higher levels of language proficiency in comparison to traditional direct instruction models because negotiating meaning in situ supports independent thinking.

The Weekend Spanish Immersion Camp aimed to negate typical obstacles found within formal classroom settings with the use of world language instructors, people who are fluent in the world language. Although not state-certified teachers, their knowledge of both the primary language students use (English) and conversational Spanish along with training from the instructor made them valuable aids in the students' efforts to learn communication skills.

The training for the world language instructors involved extensive practice in the material they would be in charge of teaching. For example, in addition to participating in planning the activities, they engaged in role plays to prepare for their supporting role and to better anticipate what the actual students would ask in certain situations. They also functioned as a constant reference who not only taught the students during the academic portion of the camp but also took part in the activities taught by outside instructors like 
cooking or dancing. This allowed for the students to have immediate help throughout each activity involving in the Spanish immersion camp.

The most important role they served was as a set of eyes and ears that observed how the individual students learned the material. We wanted our students to experience a positive and productive Spanish learning environment. Thus, at the end of each day and conclusion of each weekend session, the world language instructors and I would discuss the progress each student made and in what ways we could change our instructional methods or the teaching material to meet students' specific needs the next time they met.

Since world language instructors acted as students in camp activities, they were able to create an immersive environment with people who could not only provide students with authentic social interactions involving conversational Spanish but also immediately help along with the ability to adjust the activity to best help the student.

Providing real-time feedback and constant assessment to measure students' progress within a situated learning experience supports learners to propel knowledge development and be able to apply skills in similar situations. The opportunity to observe experts modeling the use of the language and immediately applying skills to interacting with group members who are at or similar world language level to negotiate meaning was identified by students as key to their success in the camp. This finding supports that of Dede (2009) who found that "Immersive interfaces can foster educational experiences that draw on a powerful pedagogy: situated learning" (p.66).

\section{Themes that Inform the Second Research Question}

A theme that emerged that informs the second question, What kind of activities from the Spanish immersion camp effectively support Middle School students learning a 
world language, is the Weekend Spanish Immersion Camp activities provided an opportunity to practice learning concepts. According to Stein (1998) learning is the person's capacity to apply the matter to real life situations. In keeping with Stein's work, the camp activities provided opportunities for students to put in practice what they previously learned. It was heartwarming to observe and hear our students adapting their knowledge in different situations during the camp activities. Again, analysis of the data revealed that there were four activities that were most liked by the nine students; academics, dancing, cooking, and playing games.

Based on all students' feedback, it was evident that academic activities were listed as a favorite aspect of the camp in large part due to the high student-to-instructor ratio we provided. This three-to-one ratio permitted a high level of individualized attention that intentionally build upon each students' language strengths, addressed their weaknesses, and promoted a high level of student engagement. This finding supports that of Cope, Cuthbertson, and Stoddart (2000) who indicated that one of the characteristics of learning in a practice setting is that experts or instructors can lead and support students through the complexities of task challenges. In an immersion program, experts can utilize a handful of strategies to support students' interaction and help them to obtain skills that can be used in related scenarios.

For instance, during each academic session students were asked to select the number and nature of words they wanted to learn. Language instructors then provided instruction to students on how to use those words in different tenses depending on the situation they were going to face. Students were given time to practice these words in 
different tenses in their small groups before moving to camp activities. Language instructors made sure words and verb tenses were appropriately pronounced.

After each session, the language instructors and I met to reflect on the proficiency levels of each student, reflect on students' levels of engagement in the various activities and draw on formative assessment data to adjust the lesson plans for the next day. This group reflection and planning structure allowed us to thoughtfully look for more individualized resources to support our students' oral skills in particular ways.

For students to interact properly in a given scenario they need to have the right tool to stimulate situated learning. "Tools mediate human object-oriented activity, in the sense that tools shape how we interact with reality as well as human consciousness. Tools have an objective existence independent of activity, but their function is also dependent on how they feature as parts of various activity systems" (Arnseth, 2008, p. 296). With this in mind, we placed realia intended to start or support students' interaction in each setting.

For example, the Latin chef used Spanish to instruct students on what to do and how to prepare and apply ingredients in the school kitchen that was stocked with the familiar tools of cooking such as utensils, a stove, countertop, and refrigerator. Although the chef was a native speaker of Spanish who used words and phrases unfamiliar to the students, the familiar medium of food and food preparation provided a recognizable context. The familiarity of the context helped them to quickly decode language, practice new words with manipulatives, and retain and build upon words and phrases. 
All students reported that the learning within a familiar context and doing activities they could related to helped them to feel more comfortable. Their responses were consistent with Arnseth (2008) that observed that learning is developed through interactions within an environment in recognizable contexts. To learn a world language, students need to perform and adapt a given action in different real and random scenarios.

With these ideas in mind, the dance instructor used a large, open area festooned with party decorations and speakers to recreate a ballroom. Students were encouraged to follow the instructor's commands as they danced in a setting that convincingly looked like the kind of place people would be likely to dance. Students observed that, although it felt awkward to dance with peers, repeated new and familiar words and phrases paired with movements supported comprehension and retention.

During the cooking, dancing, games, craft, academic and singing session, I observed students enjoying the activities while interacting in Spanish. Students' language interaction plays a very important role in language development. Again, students' positive responses to how social interaction promoted learning aligns with Arnseth's (2008) assertion of the fundamental importance of practice occurring within the context of social interactions. Situations wherein students must construct, modify, change and apply knowledge while having fun rather than in dry learning situations that emphasize rote memorization and recitation. "This means that concepts such as roles, identities, rules and social structures are realized in everyday activity" (Arnseth, 2008, p. 294, 295). 


\section{Themes that Inform the Third Research Question}

The following themes emerged from the data that inform the third research question; How does the Spanish immersion camp environment influence Middle School students' perspective of their world language learning process? When describing the Weekend Spanish Immersion Camp and language instructors, the students shared sentiments such as, "[it was] welcoming and [they] were nice to me". As director of this camp, it was delightful to receive this type of feedback as we wanted to create a familylike environment that would encourage students to speak comfortably and feel welcomed.

According to Stein's situated learning in adult education article (1998) socialization among students is the key element for students to naturally acquire the world language. Student's responses to the family-like environment we worked to foster aligned with Stein's (1998) work. Here, the students were able to move through the zone of proximal development (Vygotsky, 1978) without too many interventions from the language coaches as they gradually became more independent.

As socialization is an important element in the world language learning process, the language instructors were present to aid the students when needed, allowing for students to speak comfortably without fear of making mistakes. As one student stated "I didn't have to feel embarrassed". Without fear of embarrassment the students took risks, not only to improve their oral language skills at camp, but to also help their peers during the camp and in between camp sessions in class. As another student stated "when the other kids are struggling, I can help them out a little bit more because I know a little bit more after the immersion camp". 
Considering the non-traditional learning environments that the Weekend Spanish Immersion Camp structured for the students, a majority of the students' learning was achieved using a situated learning approach. According to Stein, "In the situated learning approach, knowledge and skills are learned in the contexts that reflect how knowledge is obtained and applied in everyday situations" (Stein, 1998, p. 1). This means that while students do acquire knowledge in different ways, in order to apply the new knowledge, there must be interaction in an environment to foster entrenching of new concepts within their body of knowledge to ensure retention.

All nine students reported that the Weekend Spanish Immersion Camp accomplishes this goal by combining traditional and non-traditional learning environments. The salsa class is an example of situated learning as the students were expected to make connections between vocabulary they knew and form an understanding as to how to apply their knowledge in a new environment as the salsa instructor repeatedly used known and new terms and phrases to instruct movements. For example, when the students were instructed "Gira a la derecha" they understood derecha meant right, from classroom instruction, but they did not know that gira[r] meant to spin until they saw the instructor spin. From that point on, when they heard "gira[r]" they knew that meant to spin.

I observed that while students did learn new vocabulary through the academic activities, which increased their ability to communicate, it was the non-traditional academic activities where most of their learning occurred, potentially due to the advantages of the situated learning approach. Indeed, students confirmed this 
observation throughout each hands-on activity that challenge their abilities, while fostering their language skill growth.

According to Contu and Willmott (2003) situated learning is the combination of body language, peer competitions, and knowledge management in an environment. This study aligns with that of Contu and Willmott (2003) in that I observed that being in contact with other groups of people with similar language backgrounds created an effective and easy learning environment. Indeed, the students involved in this camp cooperated to create a positive social environment for learning.

The academic and game activities, specifically, caused the students to create a competitive atmosphere for themselves. This was perhaps made all that more interesting because all of the students entered the camp on an even playing field in regards to their oral Spanish skills. The academic sessions became competitive for the students due to the way the language instructors conducted the vocabulary activities. One example is the case of a memorization game where the students chose 10-15 new vocabulary words and were given 10-minutes to memorize as many of the words that they could. When the students were tested, they quickly assumed a competitive attitude as they strived to be able to recall all their new verbs.

\section{Implications}

Here, I will discuss three big ideas related to how this Weekend Spanish Immersion Camp influenced middle school students involved in this study and implications for world language education programming; learning, behavior and 
relationships, and self-confidence. I will begin with a discussion related to student learning.

\section{Learning}

Data that emerged from this study indicates that students perceived that the immersion experience significantly improved their language fluidity including standard use of terms and pronunciation. Indeed, my observations and that of the camp instructors support this perception that students acquired fluid use of targeted new vocabulary and improved in their abilities to properly put the words together faster in increasingly complex sentences.

One student's statement echoed that of every other student when he said; "I liked learning new vocabulary because I feel like I can say more". The questions that came to my mind in response to these types of statements was "what triggers students to say "I like to learn more"?' Based on my observations, I posit that the personalized support and positive encouragement provided to each student by native Spanish speakers who each possessed a depth of knowledge of the language was key to student success and sense of empowered motivation. Although it is not always possible to provide such a high student-to- instructor ratio, it is worth stating that personalized instruction promotes student engagement and encourages students' positive attitudes towards learning and using new vocabulary.

Another implication of this study related to the theme of learning is knowing the types of learning styles present amongst each group of students. Our goal as world language educators is to provide effective approaches to support students' progression 
towards greater levels of language proficiency and to differentiate instruction to the greatest degree possible within the limitations of each setting. Immersion experiences such as the one described in this example, provide ample time to adapt instruction to meet the specific needs of individuals and the group. Working with a small group of students provided opportunities to engage students in a broader range of activities than what is typically possible to provide during short class periods involving an average of 30 students.

Another key implication of this study relates to the ways in which we designed the language immersion aspect of the camp. Each activity provided throughout the Weekend Spanish Immersion Camp was planned to entertain students while directly supporting language usage. The purpose behind providing language support through entertainment was for the students to feel comfortable in the environment and to encourage their willingness to take risks with their use of the language. To do so, within the planning of each activity, the mentors took into consideration the types of vocabulary that would be used for each activity and adjusted the vocabulary so that the students would learn. By doing so, we were able to encourage a continuous use of Spanish amongst both the instructors and the students.

For example, the dance instructor and chef were both instructed to use Spanish only while interacting with students and the other adults during the camp. The camp staff, dance instructor and chef attended a series of planning meetings prior to the start of the camp to exchange ideas and strategies to better support oral communication. We agreed to use and reuse the same vocabulary to iteratively encourage students to become 
familiar with the use of these words and phrases. We intentionally scaffolded the introduction of particular vocabulary words to be used at all times so that students naturally incorporated those new words and phrases within their existing body of knowledge. In other words, we repeatedly reinforced the plan to consistently use the new words throughout every interaction. This, ultimately, helped the students' language growth by using Piaget's concept of biological equilibrium (Fosnot, 1996). By building an assimilative and accommodative environment, through the social and educative language learning activities, the students could flourish in the Spanish immersion environment as they had the resources to communicate with the new vocabulary they learned.

Importantly, the novel nature of the experiences we provided in the camp put every child on relatively equal footing. For example, students related that they had never cooked a Latin dish prior to the cooking session with a Latin Chef as camp. Students were instructed by the chef to do tasks in the kitchen individually and in pairs, which was new to them as they were not used to hearing directions spoken in Spanish within a kitchen setting. The ambient setting of the kitchen was new to some students, but being given instructions in Spanish was definitely new to all the students. This setting encouraged students to take initiative on tasks as no one knew the language associated with cooking.

Students were asked to think in Spanish as the instructor repeatedly used the same terms and phrases to instruct their tasks. For example, when the students were instructed "mezcla azúcar con la mantequilla" they understood from classroom instruction that 
azúcar and mantequilla meant sugar and butter, but they did not know that mezcla[r] meant to mix until they saw the instructor mime the action of mixing the sugar and butter. From that point on, when the Chef said "mezcla[r]" they knew they were meant to mix ingredients together. This type of reaction occurred because, as Vygotsky stated, the spontaneous interpretation concept suggests that one develops naturally as they construct knowledge in the process of sense-making as a result of the way they interpret and assimilate perceptions "emerging from the child's own reflections on every day experience." (Fosnot, 1996, p. 18).

\section{Behavior and Relationships}

My observations, students' responses, and the meetings with language coaches support the premise that the Weekend Spanish Immersion Camp is an effective model to increase students' willingness to learn and build relationships. We endeavored to create learning environments intended to make students feel at home. In this comfortable learning environment, we intentionally fostered the development of a sense of community with the express goal to increase the students' willingness and ability to take risks and use their Spanish more freely with their peers as and language instructors.

Furthermore, the atmosphere we created at the camp was similar to a family environment wherein students had explicitly described rights and responsibilities. As one student shared "it was fun and made all of the students bond and get to know everyone else". Indeed, students confirmed that this welcoming environment encouraged them to experience a sense of partnership in the learning endeavor.

Students showed support to each other, some of them saying; "when the other kids are struggling, I can help them out a little bit more because I know a little bit more 
after the immersion camp". I have also observed that students attending the camp were more eager to support their peers during regular class because they felt empowered and had more tools to give a hand to their peers who could not attend the camp.

The implications of these results are that setting up the learning spaces in an informal language immersion program in ways that feels fun and encourage a sense of home is an effective approach for fostering supportive friendships involving a shared sense of purpose that benefits learning. In other words, creating physical and social structures that encourage students to feel comfortable to interact and build better relationships amongst and between instructors and students promotes effective socially constructive learning within situated learning environments.

Finally, the structures provided within this informal language camp setting challenged students to want to reach for the next level of language proficiency. At the end of the camp, all nine students affirmed that the Weekend Spanish Immersion Camp "helped me grow more and understand what I need to do" and they also acknowledged that "it challenged you". The implications of this feedback revolved around students' confidence, whether it be gaining self-confidence in their language skills or feeling confident with their language skills that they could help peers.

\section{Self-confidence.}

Another implication of the results relates to ways in which this type of informal language immersion experience increases students' self-confidence. Students involved in this camp made comments such as "I got time to work on what I needed to work on. I can

pronounce [words and phrases] better now. I have a friend who doesn't feel confident in 
saying it because she can't pronounce it easily, so this activity helped her and helped me because now I can say it better instead of saying it wrong”.

Another student indicated "I've liked experimenting with a lot of knowledge... I am forcing myself to practice Spanish,... in put it into... saying it”. In other words, these students expressed a sense of feeling capable of doing things that they could not do before.

This increased sense of self-confidence not only as a learner but in terms of agency as a co-learner is an excellent outcome not only in terms of the camp experience, but also in terms of continued learning in the Spanish Two classroom. Thus, an important implication is that providing this type of fun yet challenging language immersion experience early in the second year of a world language program provides benefits for improved learning outcomes throughout the academic year.

Furthermore, increased self-confidence has implications that go beyond the classroom, particularly in multilingual school settings. As one student shared "I can say more now and if my friends are trying to talk in Spanish, I can understand a lot more". Having had the experience of speaking with native Spanish speakers throughout the Spanish immersion camp, students are more comfortable in situations wherein Spanish is the main form of communication.

Next, I describe future research that would expand on the utilization of the Weekend Spanish Immersion Camp type models with different age groups and levels of experience. Here, I recommend both research examining informal education that takes place beyond the classroom setting and ways to expand formal classroom learning. 


\section{Recommendations for Future Research}

There have been very few studies conducted to date on the topic of Spanish language immersion camps in the Unites States. In particular, there is a dearth of studies focused on Spanish language immersion camps focused on second year, middle school students. Thus, there is a significant gap to fill in terms of understanding how immersion experiences serve as important supplements for formal language instruction. Here, I will provide four recommendations for future research involving world language immersion camps:

First, most public-school students begin world language studies while in high school rather than in middle school. I recommend that this camp structure be provided to second year high school level Spanish language learners, and that this study be replicated with the goal of ascertaining the perspectives of this slightly older group of learners then those involved in my study.

According to Stein (1998) learning is a person's capacity to apply new knowledge and skills to real life situations. The best way to detect if a student is learning a new language is to observe and listen to the student practicing the use of their new knowledge. With this in mind, I believe that offering this camp structure to both middle school students and older learners would provide ideal immersion experiences for students to practice and hone their oral language skills.

Secondly, I recommend that similar language immersion experiences be provided as part of college level world language teacher training programs. This approach would provide future teachers instructional modeling of an effective approach to supporting 
learning new vocabulary, phrases, and conjunctions within the context of highly individualized, fun, and comfortable learning environments. Reflections on what it is about those experiences that support language skill development could provide important insights for how to implement similar experiences in supplementary programs and into classroom setting by converting the typical classroom environment into an immersive setting. This may work particularly well in schools with longer, block period scheduling.

Third, despite the fact that there is a sizable number of native Spanish speakers in the region, I found that there was a shortage of native Spanish speakers in the local area suitable for serving the role of immersion camp language instructors. Therefore, I recommend the development of partnership programs between school districts and colleges to create World Language Educator certificate programs that involve practicum experiences wherein teacher-candidates support middle or high school world language classrooms. This would serve to increase the student-to-mentor ratio in classrooms and provide teacher-candidates with valuable, mentored teaching experiences.

Furthermore, I recommend that long-term research be conducted that follows students who have experienced a world language immersion camp during middle school all the way through college to examine what, if any, the impacts of such experiences are on their world language proficiency after being completely immersed in an environment where Spanish is the only vehicle of communication. Moreover, it would be fascinating to track students' academic achievement levels in college level language courses and examine if they continue using the target language after college. 
Finally, I recommend that partnership programs that offer supplementary immersion camps also offer symposia to the community and district school board after each camp to present action research finding and elicit ideas for what can be done to better support our students to achieve high levels of world language proficiency. I recommend that researchers be involved in planning these symposia and in collecting and synthesizing feedback to inform new ways of thinking about ideas such as evidence of stages of language acquisition and ways to differentiate instruction for various learning styles and temperaments in response to assessment data and other means to adaptively improve supplementary learning experiences.

\section{Conclusion}

It is my hope that this study contributes towards the development of a foundation of understanding necessary to support the development of world language programs. I conducted this qualitative participatory observer action research to examine middle school students' perceptions of how, if at all, they can benefit from the four Weekend Spanish Immersion Camp by increasing their oral and understanding Spanish language skills. This study demonstrates that the nine students involved in this particular program concurred that having a four-Weekend Spanish Immersion Camp reinforces students' skills in learning Spanish faster in meaningful ways and fosters deep appreciate of the language.

Here I will point out some major themes of findings; high level of motivation, confidence improvement, hands-on, fun activities, challenge, and one-on-one language coaching. The high level of motivation was proven by their willingness of returning 
every weekend to be part of the camp. Then, the use of Spanish as vehicle of communication was the evidence of confidence. Weekend Spanish Immersion Camp provided fun activities that were hands-on. It was not just about learning new words but playing dancing, cooking, crafting and singing. These fun activities challenged students learning how to use the new words and language expressions in pretending real setting. One-on-one supporting was provided during the camp. Due to the one-to-three ratio, instructors had plenty of time to support and scaffold students' Spanish language.

Furthermore, this program is proven to work well assisting students to learn a second language in an authentic way involving conversational Spanish used to negotiate meaning and solve problems. This study exemplified the benefits of going beyond the typical classroom environment and immersing students within an environment where they can explore, participate, taste, play, live the culture, and expand their world language skills to increase their confidence and proficiencies. I found Situated Learning Theory to be more supportive for this research findings.

As I conclude this work, I consider all the students who participated in the Weekend Spanish Immersion Camp and took advantage of the learning support to develop, retain, and improve their oral Spanish language skills. I feel that these students should be encouraged, respected, supported, and valued for their desire to become world language knowledgeable and better prepared to meet $21_{\text {st }}$ century world challenges and take advantage of opportunities afforded to them through their knowledge of Spanish and of Latin culture. 
All of the Weekend Spanish Immersion Camp students should feel proud of their increases in their language level and their capacity to hold a conversation in Spanish in a real setting. I believe that this participatory action research is a small, but important, contribution to construct a better world language learning environment support for our students who deserve the best education. 


\section{References}

American Association for Employment in Education (2016). Educator supply and demand in the United States: 2016 report. AAEE.

Arnseth, H. C. (2008). Activity theory and situated learning theory: contrasting views of educational practice. Pedagogy, Culture \& Society, 16(3), 289-302.

Attride-Stirling, J. (2001). Thematic networks: an analytic tool for qualitative research. Qualitative Research, 1(3), 385-405. Sage.

Baxter, P., \& Jack, S. (2008). Qualitative case study methodology: Study design and implementation for novice researchers. The Qualitative Report, 13(4), 544559.

Bialystok, E., Peets, K. F., \& Moreno, S. (2014). Producing bilinguals through immersion education: Development of metalinguistic awareness. Applied psycholinguistics, 35(01), 177-191.

Björklund, S., \& Mård-Miettinen, K. (2011). Integrating multiple languages in immersion: Swedish immersion in Finland. Immersion education: Practices, policies, possibilities, 13-35.

Burn, B. B., \& Perkins, J. A. (1980). International education in a troubled world. The ANNALS of the American Academy of Political and Social Science, 449(1), 17-30

Cammarata, L., \& Tedick, D. J. (2012). Balancing content and language in instruction: The experience of immersion teachers. The Modern Language Journal, 96(2), 251-269. 
Center for Public Education. (2011). What research says about English language learners: At a glance. Retrieved from: http://www.centerforpubliceducation.org/Main- Menu/Instruction/What-researchsays-about-English-language-learners-At-a-glance

Chastonay, P., Zesiger, V., Klohn, A., Soguel, L., Mpinga, E. K., Vu, N. V., \& Bernheim, L. (2013). Development and evaluation of a community immersion program during preclinical medical studies: a 15-year experience at the University of Geneva Medical School. Advances in medical education and practice, 4, 69.

Coghlan, D., \& Brannick, T. (2014). Doing action research in your own organization. Sage.

Contu, A., \& Willmott, H. (2003). Re-embedding situatedness: The importance of power relations in learning theory. Organization science, 14(3), 283-296.

Cope, P., Cuthbertson, P., \& Stoddart, B. (2000). Situated learning in the practice placement. Journal of advanced nursing, 31(4), 850-856.

Creswell, J. W., \& Miller, D. L. (2000). Determining validity in qualitative inquiry. Theory into practice, 39(3), 124-130.

Cummins, J. (2007). Negotiating Identities: Education for Empowerment in a Diverse Society (Second edition). Ontario, CA: California Association for Bilingual Education.

Cummins, J. (2017). Teaching for transfer in multilingual school contexts. Bilingual and multilingual education, 103-115. 
Cuseo, J. (2007). The empirical case against large class size: adverse effects on the teaching, learning, and retention of first-year students. The Journal of Faculty Development, 21(1), 5-21.

Dede, C. (2009). Immersive interfaces for engagement and learning. Science, 323(5910), 66-69.

Ennis, S. R., Ríos-Vargas, M., \& Albert, N. G. (2015). The Hispanic population: 2010; 2010 census briefs. US Census Bureau, US Department of Commerce.

Fosnot, C. T. (1996). Constructivism: Theory, perspectives, and practice. Teachers College, Columbia University.

Frawley, W. (1997). Vygotsky and Cognitive Science (Trad. cast. Vygotsky y la ciencia cognitiva. Barcelona, Paidos, 1999). Paidos Iberica Ediciones.

Gardner, D. P. (1983). A nation at risk. Washington, DC: The National Commission on Excellence in Education, US Department of Education.

Gebauer, S. K., Zaunbauer, A. C., \& Möller, J. (2013). Cross-language transfer in English immersion programs in Germany: Reading comprehension and reading fluency. Contemporary Educational Psychology,38(1), 64-74.

Granell, C. G., \& Salvador, C. C. (1994). De qué hablamos cuando hablamos de constructivismo. Cuadernos de pedagogía, 221, 8-10.

Goldman, S. (2018). Dual Language Two-Way Immersion Programs: Exploring Instructional Practices that Promote Literacy Proficiency for Spanish-Speaking English Language Learners. 
Hammer, C. S., Jia, G., \& Uchikoshi, Y. (2011). Language and literacy development of dual language learners growing up in the United States: A call for research. Child Development Perspectives, 5(1), 4-9.

Harlow, S., Cummings, R. \& Aberasturi, S. (2007). Karl Popper and Jean Piaget: A Rationale for Constructivism, The Educational Forum, 71:1, 41-48.

Kearney, E. (2010). Cultural immersion in the foreign language classroom: Some narrative possibilities. The Modern Language Journal, 94(2), 332-336.

Krathwohl, D. R. (2009). Methods of educational and social science research: The logic of methods. Waveland Press.

Lang, J. (1993). Near Immersion Results in One-Third of the Time. Mid-Atlantic Journal of Foreign Language Pedagogy, 1, 109-127.

Lave, J., \& Wenger, E. (1991). Situated learning: Legitimate peripheral participation. Cambridge University Press.

Lindholm-Leary, K., \& Block, N. (2010). Achievement in predominantly low SES/Hispanic dual language schools. International Journal of Bilingual Education and Bilingualism, 13(1), 43-60.

Lindholm-Leary, K., \& Hernández, A. (2011). Achievement and language proficiency of Latino students in dual language programmes: Native English speakers, fluent English/previous ELLs, and current ELLs. Journal of Multilingual and Multicultural Development, 32(6), 531-545.

Litaker, L. R. (1990). The design, implementation, and evaluation of a French immersion weekend. 
LoCastro, V. (1989). Large Size Classes: The Situation in Japan. Lancaster-Leeds Language Learning in Large Classes Research Project Report No. 5. University of Leeds, Leeds: Overseas Education Unit, School of Education. Eric Digest. Retrieved from ERIC Database (ED322758).

MacDonald, C. (2012). Understanding participatory action research: A qualitative research methodology option. The Canadian Journal of Action Research, 13(2), $34-50$.

Maltais, A. (2012). Student Motivation and Identity Construction in an Intensive US French Immersion Program (Doctoral dissertation, University of Ottawa).

Martin, D. J., \& Loomis, K. S. (2013). Building teachers: A constructivist approach to introducing education. Cengage Learning.

Martínez, J. (2001). La activación y mantenimiento de la motivación durante el proceso de enseñanza-aprendizaje de una lengua extranjera. Didáctica. Lengua y Literatura, 13, 237.

Maxwell, J. A. (2005). Qualitative research design: An interactive approach (2nd Ed.), Sage.

McKernan, J. (2013). Curriculum action research: A handbook of methods and resources for the reflective practitioner. Milton Park, Abington, UK Routledge.

Met, M. (1993). Foreign Language Immersion Programs. ERIC Digest.

Mohr-Schroeder, M. J., Jackson, C., Miller, M., Walcott, B., Little, D. L., Speler, L., \& Schroeder, D. C. (2014). Developing middle school students' interests in STEM 
via summer learning experiences: See Blue STEM camp. School Science and Mathematics, 114(6), 291-301.

Oregon Department of education (2013). ORS 329.452/Senate Bill 222, Guidance.

Padilla, A. M., Fan, L., Xu, X., \& Silva, D. (2013). A Mandarin/English two-way immersion program: Language proficiency and academic achievement. Foreign Language Annals, 46(4), 661-679.

Peyton, J. K. (1997). Professional Development of Foreign Language Teachers. ERIC Digest. Eric Database (EDO-FL-98-0).

Piaget, J. (1970). Structuralism. Basic Books.

Platt, J. J. (2012). A Mexico City-based immersion education program: Training mental health clinicians for practice with Latino communities. Journal of marital and family therapy, 38(2), 352-364.

Prevoo, M. J., Malda, M., Mesman, J., \& van IJzendoorn, M. H. (2015). Within-and Cross-Language Relations Between Oral Language Proficiency and School Outcomes in Bilingual Children with an Immigrant Background A Meta Analytical Study. Review of Educational Research, 0034654315584685.

Pufahl, I., \& Rhodes, N.C. (2011). Foreign language instruction in US schools: Results of a national survey of elementary and secondary schools. Foreign Language Annals, 44(2), 258-288.

Rogoff, B. (2003). The cultural nature of human development. Oxford University Press.

Rubinstein-Avila, E. (2017). Immigration and Education: What Should K-12 Teachers, 
School Administrators, and Staff Know? The Clearing House: A Journal of Educational Strategies, Issues and Ideas, 90(1), 12-17.

Snow, M. A., Padilla, A. M., \& Campbell, R. N. (1988). Patterns of second language retention of graduates of a Spanish immersion program. Applied Linguistics, 9(2), 182-197.

Spencer, M. A. (1971). Understanding Piaget: An Introduction to Children's Cognitive Development. Harper \& Row Publishers.

Stein, D. (1998). Situated Learning in Adult Education. ERIC Digest. ERIC Database (195).

Thomas, W. P., Collier, V. P., \& Abbott, M. (1993). Academic achievement through Japanese, Spanish, or French: The first two years of partial immersion. The Modern Language Journal, 77(2), 170-179.

Thomas, W., \& Collier, V. (2012). English learners for a transformed world. Albuquerque, NM: Dual Language Education of New Mexico, Fuente Press.

Trujillo, L. A. (1982). Foreign Language Camps: Jefferson County Public Schools R-1.

Valdés, G. (1995). The teaching of minority languages as academic subjects: Pedagogical and theoretical challenges. Modern Language Journal, 299-328.

Vines, L. (1983). A Guide to Language Camps in the US: 2. Language in Education: Theory and Practice, No. 53. Center for Applied Linguistics.

Vygotsky, L. (1978). La mente en la sociedad: el desarrollo de las funciones psicológicas superiores. Harvard University Press. 
Wallis, C., \& Steptoe, S. (2006). How to bring our schools out of the 20th century. Time magazine, 168(25), 50-56.

Wilson, W. H., \& Kamanā, K. (2011). Insights from Indigenous language immersion in Hawai'i. Immersion education: Practices, policies, possibilities, 36-57.

Young, M. F. (1993). Instructional design for situated learning. Educational technology research and development, 41(1), 43-58. 


\section{Appendix A: Invitation to the Research}

\section{Invitation to participate in the Research}

Dear Student and parents,

I am recruiting participants for a study about world language immersion camp as part of the requirements to complete a doctoral program in Curriculum and Instruction in the Graduate School of Education at Portland State University. The purpose of this study is to explore in depth the experiences middle school students can obtain by being immersed for four weekends in a Spanish cultural environment to support students' communication skills. The research questions are:

1. To what extent do Middle School Spanish students perceive that Spanish immersion camp instruction serves to assist retention of world language communicative skills?

2. What kind of activities from the Spanish immersion camp effectively support Middle School students learning a world language?

3. How does the Spanish immersion camp environment influence Middle School students' perspective of their world language learning process?

Your contribution to this study is valuable and important given the challenge of world language knowledge proficiency. This study aims at gaining insights from world language students participating in the immersion program. Your participation is this study is voluntary and you may withdraw from this study at any time. If you decide to participate, you will be asked to meet for four weekends. You will also be asked to complete an application package. The application form contains two pre-camp question 
that need to be answered in a paragraph format. All information you share will be kept confidential and your identity will be protected by the use of a pseudonyms.

The data will be used in partial fulfillment of doctoral research by Jorge O. Ramirez and for future publications in the area of world language education. Participation in this study implies consent for use of the results in future published research. You may choose not to participate in this study by checking the box below which states: I do not wish to participate in this study. If you decide to participate please check the box that states: I want to participate in this study. You also will need to request the application form in the school office.

I appreciate your time and consideration.

Jorge O. Ramirez

(cut)

Name: Date:

I do not wish to participate in this study

I want to participate in this study. 


\section{Appendix B: Consent and Release}

\section{Spanish Immersion Camp Consent and Release}

(This completed form must be kept on file by the teacher/researcher)

Date:

Part 1. Student Acknowledgement and Release (to be signed by student)

I know of no reason why I am not eligible to participate on the Spanish Immersion camp.

If accepted as representative, I agree to follow the rules of my school and Spanish

Immersion Camp program and to abide by their decisions. I know that participation is voluntarily. I voluntarily accept any and all responsibility for my own safety and welfare during this Spanish Immersion camp. I understand that part of my camp participation consists on:

1. Completing and submitting the pre-camp questions.

2. Being part of all activities and complete some task.

3. Being part of the interview and respond the question the best I can.

I further hereby authorize the use of disclosure of my individually identifiable health information should treatment for illness of injury become necessary. I understand that this authorization is voluntarily and that I may revoke it at any time by submitting the revocation in writing to my school. Furthermore, I grant the release parties the right to photograph, video tape or record my voice and further use my information, activity task works, voice or video tape in connection with research study, exhibitions or future camp publicity without reservation or limitation. The release parties, however, are under no obligation to exercise said rights herein.

I HAVE READ THIS CAREFULLY AND KNOW IT CONTAINS A RELEASE.

Date: Signature of Student:

Name of Student (printed):

Part 2. Parental/Guardian Consent, Acknowledgement and Release (to be completed and signed by all parents/guardians; where divorced or separated, parent/guardian with legal custody must sign).

A). I/we hereby give consent for my child/ward to participate in the Spanish Immersion camp.

B). I/we understand that participation may necessitate an early dismissal or absence from home and/or classes.

C). I/we consent to the disclosure, by my child's/ward's school, to School District, upon request, of all detailed financial, scholastic and attendance records of such school concerning my child/ward. I/we further hereby authorize the use or disclosure of my child's/ward's individually identifiable health information should treatment for illness or 
injury become necessary. I/we understand that this authorization is voluntary and that I/we may revoke it at any time by submitting the revocation in writing to my child's/ward's school.

D). I/we know of acknowledge that my child/ward knows of the risk involved in participating in this Spanish Immersion camp, understands that serious injury, and even death, is possible in such participation and choose to accept any and all responsibility for his/her safety and welfare while participating in this Spanish Immersion camp. With full understanding of the risk involved, I/we release and hold harmless my child's/ward's school and School District, it's board, employees, volunteers or another staff support of any and all responsibility and liability for any injury or claim resulting from such participation and agree to take no legal action against The School District because of any accident or mishap involving the participation of my child/ward. I further authorize emergency medical treatment for my child/ward should the need the need arise for such treatment while my child/ward is under the supervision of the school. Further, I grant the release parties the right to photograph, video tape and voice record of my child/ward and further to use said child's/ward's information, activity task works, voice or video tape in connection with research study, exhibitions or future camp publicity without reservation or limitation. The release parties, however, are under no obligation to exercise said rights herein.

E). I/we understand the I/we need to provide medical insurance number to school/ Spanish Immersion program for medical treatment when necessary.

\section{Health plan/insurance (ie. Regence, Kaiser, Providence, $\quad$ Group/policy number:} etc.):

I/WE HAVE READ THIS CAREFULLY AND KNOW IT CONTAINS A

\section{RELEASE.}

Date: Signature of Parent/Guardian:

Name of Parent/Guardian (printed):

Signature of Parent/Guardian:

Name of Parent/Guardian (printed): 


\section{Appendix C: Camp Information Package}

\section{Spanish Immersion Camp Information Package}

\section{What is the Spanish Immersion Camp?}

The Spanish Immersion Camp is a four-weekend non-traditional teaching world language to middle school students learning approach to support students' speaking skill. Spanish Immersion Camp is a safe and positive learning environment created at Neil Armstrong Middle School to support students' world language learning. The Spanish Immersion Camp is developed in four weekends during October and November 2018. The Spanish Department and school administration are always looking for approaches to better support students learning. A license teacher, alone with other native Spanish support staff, conducts the Spanish Immersion Camp.

\section{Purpose (goals) of the Spanish Immersion Camp.}

The primary purpose of the Spanish Immersion Camp is to extend learning beyond school schedule to allow students increase their Spanish communication level by been immersed in a Spanish cultural environment. Therefore, we are providing for a superior education that challenges our students to achieve academic excellence and to become more fluent in the world language.

\section{Education Benefits}

- To encourage better communication with the group and to others.

- To learn/practice Spanish in a healthy, friendly and safe environment. 
- To provide a highly supportive setting that increases student's ability to carry out daily situations independently in Spanish.

\section{Instructional Time}

The 2018 Spanish Immersion Camp is developed in weekend sessions. Each session starts at 4:00 pm on Friday and ends at 6:00 pm. on Saturday.

\begin{tabular}{|l|l|l|}
\hline Week & Start date & Ending date \\
\hline First week & Friday, October 26, 2018 & Saturday, October 27, 2018 \\
\hline Second week & Friday, November 2, 2018 & Saturday, November 3, 2018 \\
\hline Third week & Friday, November 9, 2018 & Saturday, November 10, 2018 \\
\hline Fourth week & Friday, November 16, 2018 & Saturday, November 17, 2018 \\
\hline
\end{tabular}

\section{Data collection}

Data collection will happen in three stages:

Stage 1. The first data collection will take place during application process. Applicants will answer the two questions. The questions should be written in a short paragraph format. There is not word limit on the answers. The answers to the question will be collected during application submission.

\section{Participants}

Any second-year Spanish student enrolled in 2018-2019 school year. Students should complete and submit an application form package. Only interested students will be consider for the Spanish Immersion Camp. 


\section{Lodging}

The Spanish Immersion Camp will be held at Neil Armstrong Middle School facility.

\section{Supervision}

Mr. Ramirez, Spanish Teacher, will supervise the Spanish Immersion Camp alone a group of native Spanish speaker staff support.

\section{Emergency communication}

The emergency cellphone number is 503-750-3730. Parents are encouraged to contact at any emergency situation.

\section{Student's Protocol Conduct}

I will:

1. Use Spanish only while in the camp.

2. Attend to all four sessions and do my best performance.

3. Participate in all activities prepared for this camp.

4. Be positive and display a good attitude during activities.

5. Complete all learning activities the best I can.

6. Follow the instructions of any adult supervisors, instructor or teacher at all times.

7. Use appropriate manners and respect everyone during the camp sessions.

8. Take good care of my belongings and respect other's properties.

9. Not use any electronic devise unless instructed by the staff.

10. Take part in all interview.

11. Cooperate and be honest with all information asked by adults. 


\section{Appendix D: Parent Application Form Spanish Immersion Camp Parent Application Form}

As the legal guardian for __, I wish to apply for him/her to participate in the Spanish immersion camp during October/November 2018

If is selected to be a participant in this project, I understand I will assume the obligation of providing the following: (please initial each item)

sleeping bag or cot

health/accident insurance

cellphone (for emergency use only)

I will also understand my responsibility to talk to my child about: (please initial each item)

Spanish immersion guidelines

proper manners toward adults and peers

Parent/Guardian

Date

Parent/Guardian

Date 


\section{Appendix E: Student Application Form}

\section{Spanish Immersion Camp Student Application}

$\mathrm{I}$, wish to apply for inclusion in the 2018 Spanish immersion camp.

I understand that $\mathrm{i}$ will have responsibilities before, during, and after the Spanish immersion camp. my responsibilities will include:

I will: (please initial each one) respect everyone during the Spanish immersion camp not use my cellphone unless instructed by an adult actively participate in all activities.

leaders. follow all rules established for safety of the group and as directed by the group answer and submit the pre-camp questions.

Complete all camp activities task the best I can.

Be part of the interview process

Student

Date

Parent/Guardian

Date 


\section{Appendix F: Student Information \\ Student Critical Information}

Participant's name

Address

Telephone

Mom's cell telephone

Dad's cell telephone

\section{EMERGENCY INFORMATION}

Physician Name

Office telephone

\section{INSURANCE INFORMATION}

INSURANCE COMPANY

POLICY IDENTIFICATION NUMBERS

CONTACT PHONE NUMBER

MEDICATIONS/PRESCRIPTIONS

MEDICATION

DOSAGE

MEDICATION DOSAGE

MEDICATION DOSAGE

KNOWN ALLERGIES TO MEDICATIONS:

OTHER ALLERGIES:

Parent's name:

Parent's signature: Date: 


\section{Appendix G: Pre-Camp Questionnaire}

Pre- Camp Questions

Participant code:

Date:

(please answer your question in paragraph format)

This qualitative study involves two open ended questions the interest in the Spanish Immersion Camp. Please answer the following question, giving me as many details as possible. Participation in this study is voluntarily and you may stop at any time. Turn in this paper at the moment of application submission.

1) Why do you wish to participate in this learning camp?

2) What are you expecting to see and get during this four weekend Spanish Camp? 


\section{Appendix H: Interview Protocol}

\section{Interview Protocol}

Participant Code:

Time of interview:

Date:

Place:

Interviewer: Jorge Ramirez

This qualitative study involves three open ended research questions (the answers will be recorded for accuracy answer transcribing purposes) about the experiences of Spanish Immersion Camp

Please answer the following question, giving me as many details as possible. Think about your experience in the Spanish Immersion Camp. Your answers will remain confidential and will be used for research purposes. Participation in this study is voluntarily and you may stop at any time. I will take a few notes during the interview and I will audio record this interview to transcribe it afterwards. Is it alright if I record our conversation?

1. To what extent do Middle School Spanish students perceive that Spanish immersion camp instruction serves to assist retention of world language communicative skills?

2. What kind of activities from the Spanish immersion camp effectively support Middle School students learning a world language?

3. How does the Spanish immersion camp environment influence Middle School students' perspective of their world language learning process? 


\section{Appendix I: Camp Schedule}

\section{Spanish Immersion Camp Schedule of Activities}

October/November

2018

Mr. Ramirez

Spanish teacher

\section{Objectives}

- To encourage better communication with the group and to others.

- To learn Spanish in a healthy, friendly and safe environment.

- To provide a highly supportive setting that increases the student's ability to carry out daily tasks independently in Spanish.

Instructional time: the camp will be conducted in four weekend sessions;

\begin{tabular}{|l|l|l|}
\hline \multicolumn{1}{|c|}{ Weekends } & \multicolumn{1}{c|}{ Friday } & \multicolumn{1}{c|}{ Saturday } \\
\hline First weekend & October 26th & October 27 \\
\hline Second weekend & November 2nd & November 3rd \\
\hline Third weekend & November 9th & November 10th \\
\hline Fourth weekend & November 16th & November 17th \\
\hline
\end{tabular}

Food: school will provide Food.

Housing: Middle School

Donations: Welcome!

Spanish Immersion Camp Plan

October-November 2018

1. Each weekend, the camp will start on Fridays at 4:00/30 until Saturday at $6 \mathrm{pm}$. It is expected that parents will pick up their student at 6 o'clock.

2. Each weekend, the schedule will have lots of activities.

3. Students will be divided in small groups. Each group will be led by an adult (language coach). 


\section{Friday October 26th 2018}

\begin{tabular}{|c|c|c|c|}
\hline Hour & Class & Activity & Objective/activity \\
\hline $\begin{array}{l}4: 00 \\
4: 30\end{array}$ & & $\begin{array}{l}\text { Arriving and } \\
\text { Sign in }\end{array}$ & $\begin{array}{l}\text { Students will arrive and get their camp ID and } \\
\text { Camp Pledge. }\end{array}$ \\
\hline $\begin{array}{l}4: 20 \\
4: 30\end{array}$ & & Direction & $\begin{array}{l}\text { Students will get clear directions for their } \\
\text { camp behavior and performance. Students } \\
\text { will read the camp pledge. }\end{array}$ \\
\hline $\begin{array}{l}4: 30 \\
5: 30\end{array}$ & Session 1 & Academic & $\begin{array}{l}\text { Verb use. In groups of } 3 \text { students will be } \\
\text { couched on learning verbs. }\end{array}$ \\
\hline $\begin{array}{l}5: 30 \\
6: 30\end{array}$ & Session 2 & Play & $\begin{array}{l}\text { Students will be divided assigned roles. They } \\
\text { will rehearse with their coach on their roles } \\
\text { and learn the script. After some practice, they } \\
\text { get together as a group and rehearse the play. }\end{array}$ \\
\hline $\begin{array}{l}6: 30 \\
7: 30\end{array}$ & Session 3 & Academic & $\begin{array}{l}\text { Verb use. In groups of } 3 \text { students will be } \\
\text { couched on learning verbs. }\end{array}$ \\
\hline $\begin{array}{l}7: 30 \\
8: 30\end{array}$ & & Dinner & $\begin{array}{l}\text { Students will eat a healthy dinner ordered } \\
\text { from a restaurant. }\end{array}$ \\
\hline \multirow[t]{4}{*}{$\begin{array}{l}8: 30 \\
10: 30 \\
\end{array}$} & & Games & $\begin{array}{l}\text { Students will improve their Spanish skills by } \\
\text { interacting in different activities. }\end{array}$ \\
\hline & Activity 1 & $\begin{array}{l}\text { Sentence } \\
\text { construction }\end{array}$ & $\begin{array}{l}\text { In groups of } 3 \text { students will build sentences. } \\
\text { The sentences are in a box. They have to run } \\
\text { from one station to another to accomplish the } \\
\text { task. }\end{array}$ \\
\hline & Activity 2 & $\begin{array}{l}\text { Prepare a } \\
\text { recipe }\end{array}$ & $\begin{array}{l}\text { Students will be given a list of five recipes. } \\
\text { They will write down the list of ingredients. } \\
\text { They will run to the store and get the price for } \\
\text { the ingredient. They will go back to the group } \\
\text { and get the money. They will go back to the } \\
\text { store and get the ingredient. They have to } \\
\text { collect each ingredient at a time. }\end{array}$ \\
\hline & Activity 3 & $\begin{array}{l}\text { Three tables } \\
\text { task }\end{array}$ & $\begin{array}{l}\text { Students will take turns to go through all } \\
\text { three table. Students will find a challenge on } \\
\text { each table they have to accomplish. }\end{array}$ \\
\hline $\begin{array}{l}10: 30 \\
11: 30\end{array}$ & & Movie & $\begin{array}{l}\text { Students will watch a PG movie in the target } \\
\text { language to improve their listening skill. }\end{array}$ \\
\hline 11:30 & & Bedtime & $\begin{array}{l}\text { There will be two sleeping areas (boys and } \\
\text { girls). }\end{array}$ \\
\hline
\end{tabular}




\section{Saturday October 27th 2018}

\begin{tabular}{|c|c|c|c|}
\hline Hour & Class & Activity & Objective/activity \\
\hline $\begin{array}{l}8: 00 \\
9: 00\end{array}$ & & Breakfast & $\begin{array}{l}\text { Students will eat a healthy breakfast in the } \\
\text { lunchroom. }\end{array}$ \\
\hline $\begin{array}{l}9: 00 \\
10: 00\end{array}$ & Session 1 & Academic & $\begin{array}{l}\text { Verb use. In groups of } 3 \text { students will be } \\
\text { couched on learning verbs. }\end{array}$ \\
\hline $\begin{array}{l}10: 00 \\
11: 00\end{array}$ & Session 2 & Dance & $\begin{array}{l}\text { Students will execute direction on how to } \\
\text { salsa from instructor. }\end{array}$ \\
\hline $\begin{array}{l}11: 00 \\
12: 00\end{array}$ & Session 3 & $\begin{array}{l}\text { craft/ } \\
\text { cooking }\end{array}$ & $\begin{array}{l}\text { Students will work on preparing the } \\
\text { background of the stage, the houses for the } \\
\text { little pigs and masks for the play. } \\
\text { Students will assist a chef on preparing the } \\
\text { meal. Students will explain the recipe the } \\
\text { audience. }\end{array}$ \\
\hline $\begin{array}{l}12: 00 \\
1: 00\end{array}$ & & Lunch & $\begin{array}{l}\text { Students will enjoy the meal prepared by a } \\
\text { group of students and guided by a chef. } \\
\text { Students will write the recipe and explain } \\
\text { the group }\end{array}$ \\
\hline $\begin{array}{l}1: 00 \\
2: 00\end{array}$ & Session 4 & $\begin{array}{l}\text { Craft for the } \\
\text { party }\end{array}$ & $\begin{array}{l}\text { Students will be immersed into the Hispanic } \\
\text { culture about celebration and costumes. } \\
\text { Students will prepare piñata and decorations } \\
\text { that will be hung. }\end{array}$ \\
\hline $\begin{array}{l}2: 00 \\
3: 00\end{array}$ & Session 5 & Play & $\begin{array}{l}\text { Students will be divided assigned roles. They } \\
\text { will rehearse with their coach on their roles } \\
\text { and learn the script. After some practice, } \\
\text { they get together as a group and rehearse } \\
\text { the play. }\end{array}$ \\
\hline $\begin{array}{l}3: 00 \\
4: 00\end{array}$ & Session 6 & Academic & $\begin{array}{l}\text { Food/adjective vocabulary. Students will } \\
\text { increase their food vocabulary by describing } \\
\text { the food. They will also learn adjectives. }\end{array}$ \\
\hline $\begin{array}{l}4: 00 \\
5: 00\end{array}$ & Session 7 & Singing & $\begin{array}{l}\text { Students will read, understand and sing the } \\
\text { lyrics of Hispanic songs. }\end{array}$ \\
\hline $\begin{array}{l}5: 00 \\
6: 00\end{array}$ & Session 8 & $\begin{array}{l}\text { Writing } \\
\text { session }\end{array}$ & $\begin{array}{l}\text { Closure, students will get together in small } \\
\text { groups and talk about what they have } \\
\text { learned this weekend. They will state what } \\
\text { they like the most and least. After the oral } \\
\text { interaction they will answer some questions } \\
\text { in English for the research project. }\end{array}$ \\
\hline
\end{tabular}




\section{Friday November 2nd 2018}

\begin{tabular}{|c|c|c|c|}
\hline Hour & Class & Activity & Objective /activity \\
\hline $\begin{array}{l}4: 00 \\
4: 20\end{array}$ & & $\begin{array}{l}\text { Arriving and } \\
\text { Sign in }\end{array}$ & $\begin{array}{l}\text { Students will arrive and get their camp ID and } \\
\text { Camp Pledge. }\end{array}$ \\
\hline $\begin{array}{l}4: 20 \\
4: 30\end{array}$ & & Direction & $\begin{array}{l}\text { Students will get clear directions for their } \\
\text { camp behavior and performance. Students } \\
\text { will read the camp pledge. }\end{array}$ \\
\hline $\begin{array}{l}4: 30 \\
5: 30\end{array}$ & Session 1 & Academic & $\begin{array}{l}\text { Verb use. In groups of } 3 \text { students will be } \\
\text { couched on learning verbs. }\end{array}$ \\
\hline $\begin{array}{l}5: 30 \\
6: 30\end{array}$ & Session 2 & Games & $\begin{array}{l}\text { Students will be divided in groups. They will } \\
\text { create a short story using the selected verbs. }\end{array}$ \\
\hline $\begin{array}{l}6: 30 \\
7: 30\end{array}$ & Session 3 & Academic & $\begin{array}{l}\text { Verb use. In groups of } 3 \text { students will be } \\
\text { couched on learning verbs. }\end{array}$ \\
\hline $\begin{array}{l}7: 30 \\
8: 30\end{array}$ & & Dinner & $\begin{array}{l}\text { Students will eat a healthy dinner ordered } \\
\text { from a restaurant. }\end{array}$ \\
\hline \multirow[t]{4}{*}{$\begin{array}{l}8: 30 \\
10: 30 \\
\end{array}$} & & Games & $\begin{array}{l}\text { Students will improve their Spanish skills by } \\
\text { interacting in different activities. }\end{array}$ \\
\hline & Activity 1 & Word search & $\begin{array}{l}\text { Students will run to a table where they will } \\
\text { find a sheet of a word search. It is a } 10 \text {-word } \\
\text { search task. Each student will circle only one } \\
\text { word and go back to the group for the next } \\
\text { student to go and do the same task. }\end{array}$ \\
\hline & Activity 2 & Charades & $\begin{array}{l}\text { Students will be given a list of ten verbs. One } \\
\text { student will act out the verb and the other } \\
\text { two will guess the action. Once the word is } \\
\text { guessed they have to be replaced by another } \\
\text { student until the ten verbs are guessed. The } \\
\text { team will be timed. The group that finishes } \\
\text { the activity in less time wins the contest. }\end{array}$ \\
\hline & Activity 3 & kiwi & $\begin{array}{l}\text { Two team competition. One team will home } \\
\text { and the other the challenger. Each team will } \\
\text { have } 5 \text { minutes to put the cups in each place. }\end{array}$ \\
\hline $\begin{array}{l}10: 30 \\
11: 30 \\
\end{array}$ & & Movie & $\begin{array}{l}\text { Students will watch a PG movie in the target } \\
\text { language to improve their listening skill. }\end{array}$ \\
\hline $11: 30$ & & Bedtime & $\begin{array}{l}\text { There will be two sleeping areas (boys and } \\
\text { girls). }\end{array}$ \\
\hline
\end{tabular}




\section{Saturday November 3rd 2018}

\begin{tabular}{|l|l|l|l|}
\hline Hour & Class & Activity & Objective /activity \\
\hline $\begin{array}{l}8: 00 \\
9: 00\end{array}$ & & Breakfast & $\begin{array}{l}\text { Students will eat a healthy breakfast in the } \\
\text { lunchroom. }\end{array}$ \\
\hline $\begin{array}{l}9: 00 \\
10: 00\end{array}$ & Session 1 & Academic & $\begin{array}{l}\text { Verb use. In groups of 3 students will be } \\
\text { couched on learning verbs. }\end{array}$ \\
\hline $\begin{array}{l}10: 00 \\
11: 00\end{array}$ & Session 2 & Dance & $\begin{array}{l}\text { Students will execute direction on how to } \\
\text { salsa from instructor. }\end{array}$ \\
\hline $\begin{array}{l}11: 00 \\
12: 00\end{array}$ & Session 3 & craft/ & $\begin{array}{l}\text { Students will work on preparing pinatas for } \\
\text { the party. } \\
\text { Students will assist a chef on preparing the } \\
\text { meal. Students will explain the recipe the } \\
\text { audience. }\end{array}$ \\
\hline $\begin{array}{l}12: 00 \\
1: 00\end{array}$ & & Lunch & $\begin{array}{l}\text { Students will enjoy the meal prepared by a } \\
\text { group of students and guided by a chef. } \\
\text { Students will write the recipe and explain } \\
\text { the group }\end{array}$ \\
\hline $\begin{array}{l}\text { 1:00 } \\
2: 00\end{array}$ & Session 4 & Singing & $\begin{array}{l}\text { Students will read, understand and sing the } \\
\text { lyrics of Hispanic songs. }\end{array}$ \\
\hline $\begin{array}{l}2: 00 \\
3: 00\end{array}$ & Session 5 & $\begin{array}{l}\text { Craft for the } \\
\text { party }\end{array}$ & $\begin{array}{l}\text { Students will be immersed into the Hispanic } \\
\text { culture about celebration and costumes. } \\
\text { Students will prepare piñata and decorations } \\
\text { that will be hung. }\end{array}$ \\
\hline $\begin{array}{l}\text { 3:00 } \\
4: 00\end{array}$ & Session 6 & Academic & $\begin{array}{l}\text { Food/adjective vocabulary. Students will } \\
\text { increase their food vocabulary by describing } \\
\text { the food. They will also learn adjectives. }\end{array}$ \\
\hline $\begin{array}{l}4: 00 \\
5: 00\end{array}$ & Session 7 & $\begin{array}{l}\text { Writing } \\
\text { session }\end{array}$ & $\begin{array}{l}\text { Closure, students will get together in small } \\
\text { groups and talk about what they have } \\
\text { learned this weekend. They will state what } \\
\text { they like the most and least. After the oral } \\
\text { interaction they will answer some questions } \\
\text { in English for the research project. }\end{array}$ \\
\hline
\end{tabular}




\section{Friday November 9th 2018}

\begin{tabular}{|c|c|c|c|}
\hline Hour & Class & Activity & Objective/activity \\
\hline $\begin{array}{l}4: 00 \\
4: 20\end{array}$ & & $\begin{array}{l}\text { Arriving and } \\
\text { Sign in }\end{array}$ & Students will arrive to camp and sing in \\
\hline $\begin{array}{l}4: 20 \\
4: 30\end{array}$ & & Direction & $\begin{array}{l}\text { Students will get clear directions for their } \\
\text { camp behavior and performance. Students } \\
\text { will read the camp pledge. }\end{array}$ \\
\hline $\begin{array}{l}4: 30 \\
5: 30\end{array}$ & Session 1 & Academic & $\begin{array}{l}\text { Verb use. In groups of } 3 \text { students will be } \\
\text { couched on learning verbs. }\end{array}$ \\
\hline $\begin{array}{l}5: 30 \\
6: 30\end{array}$ & Session 2 & Games & $\begin{array}{l}\text { Students will be divided in groups. They will } \\
\text { create a short story using the selected verbs } \\
\text { and present the story verbally to the group. }\end{array}$ \\
\hline $\begin{array}{l}6: 30 \\
7: 30\end{array}$ & Session 3 & Academic & $\begin{array}{l}\text { New vocabulary. In groups of } 3 \text { students will } \\
\text { be couched on learning how to use the new } \\
\text { vocabulary in context. Students will use a } \\
\text { badge with selected words on it. They will use } \\
\text { their words in conversation. }\end{array}$ \\
\hline $\begin{array}{l}7: 30 \\
8: 30\end{array}$ & & Dinner & $\begin{array}{l}\text { Students will eat a healthy dinner ordered } \\
\text { from a restaurant. }\end{array}$ \\
\hline \multirow[t]{4}{*}{$\begin{array}{l}8: 30 \\
10: 30\end{array}$} & & Games & $\begin{array}{l}\text { Students will improve their Spanish skills by } \\
\text { interacting in different activities. }\end{array}$ \\
\hline & Activity 1 & Word search & $\begin{array}{l}\text { Students will run to three different tables } \\
\text { where they will find a sheet of a word search } \\
\text { in each table. It is a } 10 \text {-word search task. Each } \\
\text { student will circle only one word and go back } \\
\text { to the group for the next student to go and do } \\
\text { the same task. }\end{array}$ \\
\hline & Activity 2 & Charades & $\begin{array}{l}\text { Students will pick a word from a list of ten } \\
\text { categories. The participant will act out the } \\
\text { word and the group will guess the name. The } \\
\text { team will be timed. The group that finishes } \\
\text { the activity in less time wins the contest. }\end{array}$ \\
\hline & Activity 3 & 7 pecados & Student will learn a new Hispanic street game. \\
\hline $\begin{array}{l}10: 30 \\
11: 30\end{array}$ & & Movie & $\begin{array}{l}\text { Students will watch a PG movie in the target } \\
\text { language to improve their listening skill. }\end{array}$ \\
\hline $11: 30$ & & Bedtime & $\begin{array}{l}\text { There will be two sleeping areas (boys and } \\
\text { girls). }\end{array}$ \\
\hline
\end{tabular}




\section{Saturday November 10th 2018}

\begin{tabular}{|l|l|l|l|}
\hline Hour & Class & Activity & Objective/activity \\
\hline $\begin{array}{l}8: 00 \\
9: 00\end{array}$ & & Breakfast & $\begin{array}{l}\text { Students will eat a healthy breakfast in the } \\
\text { lunchroom. }\end{array}$ \\
\hline $\begin{array}{l}9: 00 \\
10: 00\end{array}$ & Session 1 & Academic & $\begin{array}{l}\text { Verb use. In groups of 3 students will be } \\
\text { couched on learning verbs. Today they will } \\
\text { use the three tenses. }\end{array}$ \\
\hline $\begin{array}{l}10: 00 \\
11: 00\end{array}$ & Session 2 & Dance & $\begin{array}{l}\text { Students will execute direction on how to } \\
\text { salsa from instructor. }\end{array}$ \\
\hline $\begin{array}{l}11: 00 \\
11: 30\end{array}$ & Session 3 & Academic & Practice of food vocabulary. \\
\hline $\begin{array}{l}11: 30 \\
12: 00\end{array}$ & Session 3 & cooking & $\begin{array}{l}\text { Students will assist a chef on preparing the } \\
\text { meal. Students will explain the recipe the } \\
\text { audience. }\end{array}$ \\
\hline $\begin{array}{l}12: 00 \\
1: 00\end{array}$ & & Lunch & $\begin{array}{l}\text { Students will enjoy the meal prepared by a } \\
\text { group of students and guided by a chef. } \\
\text { Students will write the recipe and explain } \\
\text { the group }\end{array}$ \\
\hline $\begin{array}{l}1: 00 \\
2: 00\end{array}$ & Session 4 & Academic & $\begin{array}{l}\text { Food/adjective vocabulary. Students will } \\
\text { increase their food vocabulary by describing } \\
\text { the food. They will also learn adjectives. }\end{array}$ \\
\hline $\begin{array}{l}\text { 2:00 } \\
3: 00\end{array}$ & Session 5 & Singing & $\begin{array}{l}\text { Students will read, understand and sing the } \\
\text { lyrics of Hispanic songs. }\end{array}$ \\
\hline $\begin{array}{l}3: 00 \\
4: 00\end{array}$ & Session 6 & $\begin{array}{l}\text { Craft for the } \\
\text { party }\end{array}$ & $\begin{array}{l}\text { Students will be immersed into the Hispanic } \\
\text { culture about celebration and costumes. } \\
\text { Students will prepare piñata and decorations } \\
\text { that will be hung. }\end{array}$ \\
\hline $\begin{array}{l}\text { Closure, students will get together in small } \\
\text { groups and talk about what they have } \\
\text { learned this weekend. They will state what } \\
\text { they like the most and least. After the oral } \\
\text { interaction they will answer some questions } \\
\text { in English for the research project. }\end{array}$ \\
\hline Session 7 & Writing \\
session & & & \\
\hline
\end{tabular}




\section{Friday November 16th 2018}

\begin{tabular}{|c|c|c|c|}
\hline Hour & Class & Activity & Objective/activity \\
\hline $\begin{array}{l}4: 00 \\
4: 20\end{array}$ & & $\begin{array}{l}\text { Arriving and } \\
\text { Sign in }\end{array}$ & $\begin{array}{l}\text { Students will arrive and get their camp ID and } \\
\text { Camp Pledge. }\end{array}$ \\
\hline $\begin{array}{l}4: 20 \\
4: 30\end{array}$ & & Direction & $\begin{array}{l}\text { Students will get clear directions for their } \\
\text { camp behavior and performance. Students } \\
\text { will read the camp pledge. }\end{array}$ \\
\hline $\begin{array}{l}4: 30 \\
5: 30\end{array}$ & Session 1 & Academic & $\begin{array}{l}\text { Verb use. In groups of } 3 \text { students will be } \\
\text { couched on learning verbs. }\end{array}$ \\
\hline $\begin{array}{l}5: 30 \\
6: 30\end{array}$ & Session 2 & games & $\begin{array}{l}\text { Reading comprehension-competition. } \\
\text { Students will read a story and after } \\
\text { comprehend the plot they will be challenge to } \\
\text { answer question in Spanish. }\end{array}$ \\
\hline $\begin{array}{l}6: 30 \\
7: 30\end{array}$ & Session 3 & Academic & $\begin{array}{l}\text { Verb use. In groups of } 3 \text { students will be } \\
\text { couched on learning verbs. }\end{array}$ \\
\hline $\begin{array}{l}7: 30 \\
8: 30\end{array}$ & & Dinner & $\begin{array}{l}\text { Students will eat a healthy dinner ordered } \\
\text { from a restaurant. }\end{array}$ \\
\hline \multirow[t]{3}{*}{$\begin{array}{l}8: 30 \\
9: 30\end{array}$} & & Games & $\begin{array}{l}\text { Students will improve their Spanish skills by } \\
\text { interacting in different activities. }\end{array}$ \\
\hline & Activity 1 & $\begin{array}{l}\text { Prepare a } \\
\text { recipe }\end{array}$ & $\begin{array}{l}\text { Students will be given the task to recreate the } \\
\text { ingredient list of the dishes they helped } \\
\text { prepare the last weekend. They will announce } \\
\text { the list of ingredients in alphabetical order. }\end{array}$ \\
\hline & Activity 2 & $\begin{array}{l}\text { Sentence } \\
\text { construction }\end{array}$ & $\begin{array}{l}\text { In groups of } 3 \text { students will build sentences. } \\
\text { The sentences are in a box. They have to run } \\
\text { from one station to another to accomplish the } \\
\text { task. }\end{array}$ \\
\hline $\begin{array}{l}9: 30 \\
10: 30 \\
\end{array}$ & Session 4 & Craft & $\begin{array}{l}\text { Students will work on creating decoration for } \\
\text { the party. }\end{array}$ \\
\hline $\begin{array}{l}10: 30 \\
11: 30\end{array}$ & & Movie & $\begin{array}{l}\text { Students will watch a PG movie in the target } \\
\text { language to improve their listening skill. }\end{array}$ \\
\hline $11: 30$ & & Bedtime & $\begin{array}{l}\text { There will be two sleeping areas (boys and } \\
\text { girls). }\end{array}$ \\
\hline
\end{tabular}




\section{Saturday November 17th 2018}

\begin{tabular}{|c|c|c|c|}
\hline Hour & Class & Activity & Objective/activity \\
\hline $\begin{array}{l}8: 00 \\
9: 00\end{array}$ & & Breakfast & $\begin{array}{l}\text { Students will eat a healthy breakfast in the } \\
\text { lunchroom. }\end{array}$ \\
\hline $\begin{array}{l}9: 00 \\
10: 00\end{array}$ & Session 1 & Academic & $\begin{array}{l}\text { Food vocabulary use. In groups of } 3 \text { students } \\
\text { will be couched on learning verbs. }\end{array}$ \\
\hline $\begin{array}{l}10: 00 \\
11: 00\end{array}$ & Session 2 & Dance & $\begin{array}{l}\text { Students will execute direction on how to } \\
\text { salsa from instructor. }\end{array}$ \\
\hline $\begin{array}{l}11: 00 \\
11: 30\end{array}$ & Session 1 & Academic & $\begin{array}{l}\text { Clothes vocabulary use. In groups of } 3 \\
\text { students will be couched on learning verbs. }\end{array}$ \\
\hline $\begin{array}{l}11: 00 \\
12: 00\end{array}$ & Session 3 & Cooking & $\begin{array}{l}\text { Students will assist a chef on preparing the } \\
\text { meal. Students will explain the recipe the } \\
\text { audience. }\end{array}$ \\
\hline $\begin{array}{l}12: 00 \\
1: 00\end{array}$ & & Lunch & $\begin{array}{l}\text { Students will enjoy the meal prepared by a } \\
\text { group of students and guided by a chef. } \\
\text { Students will write the recipe and explain } \\
\text { the group }\end{array}$ \\
\hline \multirow[t]{2}{*}{$\begin{array}{l}1: 00 \\
2: 00\end{array}$} & Session 4 & $\begin{array}{l}\text { Party } \\
\text { decoration }\end{array}$ & $\begin{array}{l}\text { Students will be immersed into the Hispanic } \\
\text { culture about celebration and costumes. } \\
\text { Students will prepare piñata and decorations } \\
\text { that will be hung. }\end{array}$ \\
\hline & Session 8 & $\begin{array}{l}\text { Writing } \\
\text { session }\end{array}$ & $\begin{array}{l}\text { Closure, students will get together in small } \\
\text { groups and talk about what they have } \\
\text { learned this weekend. They will state what } \\
\text { they like the most and least. After the oral } \\
\text { interaction they will answer some questions } \\
\text { in English for the research project. }\end{array}$ \\
\hline $\begin{array}{l}2: 00 \\
3: 00\end{array}$ & Session 5 & Interviews & $\begin{array}{l}\text { Students will be divided assigned roles. They } \\
\text { will rehearse with their coach on their roles } \\
\text { and learn the script. After some practice, } \\
\text { they get together as a group and rehearse } \\
\text { the play. }\end{array}$ \\
\hline $\begin{array}{l}3: 00 \\
4: 00\end{array}$ & Session 6 & Party & $\begin{array}{l}\text { Students will enjoy fun activities. The } \\
\text { activities will be charades, family feud, and } \\
\text { others }\end{array}$ \\
\hline $\begin{array}{l}4: 00 \\
5: 00\end{array}$ & Session 7 & Party & $\begin{array}{l}\text { Students will dance and have conversation } \\
\text { about the camp. }\end{array}$ \\
\hline
\end{tabular}

\section{Mr. Ramirez}

\section{Spanish teacher}

\section{Spanish Immersion Camp Director}

